\title{
Retrospective evaluation of whole exome and genome mutation calls in 746 cancer samples
}

\author{
Matthew H. Bailey (10 1,2,3, William U. Meyerson 4,5, Lewis Jonathan Dursi (iD 6,7, Liang-Bo Wang (1) 1,2,

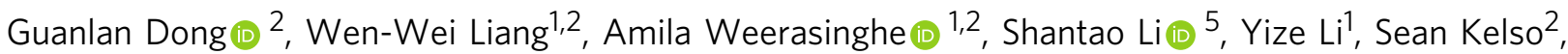 \\ MC3 Working Group*, PCAWG novel somatic mutation calling methods working group*, Gordon Saksena (1) ${ }^{8}$, \\ Kyle Ellrott [1] 9, Michael C. Wend1 170,11, David A. Wheeler (10) ${ }^{12,13}$, Gad Getz (1) 8,14,15,16, Jared T. Simpson ${ }^{6,17}$, \\ Mark B. Gerstein (1D ${ }^{5,18,19 凶}$, Li Ding ${ }^{1,2,3,20 凶}$ \& PCAWG Consortium*
}

The Cancer Genome Atlas (TCGA) and International Cancer Genome Consortium (ICGC) curated consensus somatic mutation calls using whole exome sequencing (WES) and whole genome sequencing (WGS), respectively. Here, as part of the ICGC/TCGA Pan-Cancer Analysis of Whole Genomes (PCAWG) Consortium, which aggregated whole genome sequencing data from 2,658 cancers across 38 tumour types, we compare WES and WGS side-by-side from 746 TCGA samples, finding that $~ 80 \%$ of mutations overlap in covered exonic regions. We estimate that low variant allele fraction $(\mathrm{VAF}<15 \%)$ and clonal heterogeneity contribute up to $68 \%$ of private WGS mutations and $71 \%$ of private WES mutations. We observe that $30 \%$ of private WGS mutations trace to mutations identified by a single variant caller in WES consensus efforts. WGS captures both $~ 50 \%$ more variation in exonic regions and un-observed mutations in loci with variable GC-content. Together, our analysis highlights technological divergences between two reproducible somatic variant detection efforts.

\footnotetext{
${ }^{1}$ The McDonnell Genome Institute at Washington University, St. Louis, MO 63108, USA. ${ }^{2}$ Division of Oncology, Department of Medicine, Washington University School of Medicine, St. Louis, MO 63108, USA. ${ }^{3}$ Alvin J. Siteman Cancer Center, Washington University School of Medicine, St. Louis, MO 63108, USA. ${ }^{4}$ Yale School of Medicine, Yale University, New Haven, CT 06520, USA. ${ }^{5}$ Program in Computational Biology and Bioinformatics, Yale University, New Haven, CT 06520, USA. ${ }^{6}$ Computational Biology Program, Ontario Institute for Cancer Research, Toronto, ON M5G 0A3, Canada. ${ }^{7}$ The Hospital for Sick Children, Toronto, ON M5G 1X8, Canada. ${ }^{8}$ Broad Institute of MIT and Harvard, Cambridge, MA 02142, USA. ${ }^{9}$ Biomedical Engineering, Oregon Health and Science University, Portland, OR 97239, USA. ${ }^{10}$ Department of Mathematics, Washington University in St. Louis, St. Louis, MO 63130, USA. ${ }^{11}$ Department of Genetics, Washington University School of Medicine, St.Louis, MO 63110, USA. ${ }^{2}$ Human Genome Sequencing Center, Baylor College of Medicine, Houston, TX 77030, USA. ${ }^{13}$ Department of Molecular and Human Genetics, Baylor College of Medicine, Houston, TX 77030, USA. ${ }^{14}$ Harvard Medical School, Boston, MA 02115, USA. ${ }^{15}$ Center for Cancer Research, Massachusetts General Hospital, Boston, MA 02114, USA. ${ }^{16}$ Department of Pathology, Massachusetts General Hospital, Boston, MA 02114, USA. ${ }^{17}$ Department of Computer Science, University of Toronto, Toronto, ON M5S, Canada. ${ }^{18}$ Department of Computer Science, Yale University, New Haven, CT 06520, USA. ${ }^{19}$ Department of Molecular Biophysics and Biochemistry, Yale University, New Haven, CT 06520, USA. ${ }^{20}$ Department of Medicine and Department of Genetics, Washington University School of Medicine, St. Louis, MO 63110, USA. *Lists of authors and their affiliations appear at the end of the paper.凶email: mark.gerstein@yale.edu; Iding@wustl.edu
} 
C omplementary efforts of The Cancer Genome Atlas (TCGA) and the International Cancer Genome Consortium (ICGC) have recently produced two of the highest quality and most elaborate and reproducible somatic variant call sets from exome and whole genome-level data in cancer genomics, respectively. The motivation for these efforts stems from the notion that "scientific crowd sourcing" and combining mutation callers can provide very strong results.

These two efforts produced variant calls from 10 different callers, namely Radia ${ }^{1}, \operatorname{Varscan}^{2}, \mathrm{MuSE}^{3}, \mathrm{MuTect}^{4}, \mathrm{Pindel}^{5,6}$, Indelocator $^{7}$, SomaticSniper ${ }^{8}$ for WES and MuSE, BroadPipeline (anchored by MuTect), Sanger-pipeline, German Cancer Research Center pipeline (DKFZ), and $\mathrm{SMuFin}^{9}$, for WGS. Briefly, the PCAWG Consortium aggregated whole genome sequencing data from 2658 cancers across 38 tumor types generated by the ICGC and TCGA projects. These sequencing data were re-analyzed with standardized, highaccuracy pipelines to align to the human genome (reference build hs37d5) and identify germline variants and somatically acquired mutations ${ }^{10}$. Of the 885 TCGA samples in ICGC, 746 were included in the latest exome call set produced by both the Multi-Center Mutation Calling in Multiple Cancers (MC3) effort and the Pan-Cancer Analysis of Whole Genomes (PCAWG) Consortium set. These 746 samples represent a critical benchmark for high-level analysis of similarities and differences between exome and genome somatic variant detection methods.

Reproducibility of mutations identified by both whole exome capture sequencing and whole genome sequencing (WGS) techniques remains an important issue, not only for the scientific use of large, established data sets, but for data designs of future research projects. Previous work analyzing exome capture effects on sequence read quality has shown that GC-content bias is the major source of variation in coverage ${ }^{11}$. A performance comparison across exome-captured platforms demonstrated that for most technologies, both high and low GC-content result in reduced coverage in read depth ${ }^{12}$. Belkadi et al. compared mutation calls between WGS and WES, observing that $~ 3 \%$ of coding variants with high quality were only detected in WGS, and WGS also had a more uniform distribution of coverage depth, genotype quality, and minor read ratio ${ }^{13}$. Furthermore, due to the relatively high error rate per read in next-generation sequencing ${ }^{14}$, the detectability of mutations with low variant allele fractions (VAFs) is limited by background noise. Despite these studies' nuanced preference towards WGS, others contend that WES will remain a better choice until costs of WGS fall ${ }^{15}$. The decision to sequence exomes or whole genomes is further confounded as more recent publications in oncology select either WGS ${ }^{16-20}$ or WES ${ }^{21-24}$. Recognizing the unresolved nature of this issue, Schwarze et al. have called for more comprehensive studies comparing the WES and WGS studies, especially as this issue has important ramifications for the clinic $^{25}$.

Our analysis provides confidence that mutation calls within the captured exonic regions of these two data sets are largely consistent. We highlight common sample, cohort, and caller-specific challenges in cancer variant detection from the TCGA and ICGC efforts. We show that variants that are most confidently called in one database i.e., called by multiple callers, are very likely to be called in the other. We assess how reproducibility impacts higher-level mutation signature analysis and illustrate the need for caution in assessing performance that can only be identified by the overlap of these two data sets. Finally, we explore the capacity of WGS to detect recurrent non-coding mutations captured by whole exome sequencing.

\section{Results}

Data and workflow. We used publicly available data from the MC3 and PCAWG repositories, consisting of $\sim 3.6 \mathrm{M}$ and $\sim 47 \mathrm{M}$ variants, respectively (Fig. 1a). 746 samples were sequenced by both WES and WGS, comprising various aliquots and portions of the same tumor (Supplementary Data 1, Fig. 1b). Effects of these differences are discussed below for preliminary results, but we ultimately used the entire set of 746 samples in the variant overlap analysis, since the effects of tumor partitioning did not play a significant role (Supplementary Fig. 1). By restricting the public data sets to overlapping samples, we reduced the total corpus to $\sim 220 \mathrm{~K}(6.1 \%)$ and $\sim 23 \mathrm{M}(49.6 \%)$ mutations for exome and whole genome, respectively. It is notable that there is an enrichment of variants in hypermutated samples from COAD, HNSC, LUAD, and STAD in the PCAWG set used in this study (Supplementary Fig. 2). To begin building a comparable set of mutations between these two studies, we further restricted the whole genome data set to exon regions provided by the MC3 analysis working group. This reduced the WGS data set to $1.6 \%$ of its original size, within range of total exome material estimations $^{26}$ (Fig. 1a). The next step involved removing poorly-covered variants potentially caused by technical anomalies by limiting mutations to those captured in coverage files (distributed as.wig files). A reciprocal coverage strategy was used, meaning PCAWG mutations were restricted to covered genomic regions in MC3 and vice versa, thereby maintaining a complementary set of callable genomic regions. Removal of mutations in uncovered regions reduced the remaining PCAWG data set by approximately one-half, from 387,166 to 183,424 mutations. We also identified 4241 MC3 and 2219 PCAWG mutations that were present in the respective MAF but were not marked as covered in the coverage files provided by a single group. This suggests that different tools consider different minimum coverage strategies. These mutations reflect $2.0 \%$ and $1.2 \%$, respectively, of the total mutation discrepancy and were removed because some callers had limited capacity to identify mutations in poorly-covered regions (see "Methods" section). Finally, filter flags provided by MC3 were used to assess somatic mutation filtering strategies. At this stage, we performed filter optimization to comprehensively evaluate all possible combinations of MC3 filters (Supplementary Fig. 3a). Ultimately, we decided to only remove OxoG labeled artifacts and duplicated events produced by these filters (see "Methods" section, Supplementary Data 2). Since each stage of this filtering workflow resulted in many alternative decisions and outcomes, we built MAFit, a web-based graphical user interface that allows users to easily customize comparisons of merged mutations (https://mbailey.shinyapps.io/MAFit/). A MC3 filter assessment also shows that many variants with filter flags in MC3 are present in the PCAWG variant call set, suggesting a need for improved filtering strategies (Supplementary Fig. 3b).

TCGA samples comprise a sizable fraction of the PCAWG sample pool ( $30 \%$, Supplementary Data 1$)$ Additional WGS sequencing from TCGA allowed for mutation validation ${ }^{27}$ and insights into non-coding mutations, such as in TET2. However, this selection process could have potentially influenced our basic comparison of exome-sequenced samples and genome-sequenced samples in two fundamental ways. First, vagaries of tumor extraction and tissue storage protocols may have resulted in many different portions of a tumor being stored, introducing the possibility that different subclones of the same tumor could be present. These could have very different genetic makeups. This information was captured in different substrings of the TCGA identification barcode (see "Methods" section). From the 746 TCGA barcodes, we found that $64 \%$ (477) could be traced to the same well of a microtiter plate (Fig. 1b). After correcting for cancer type, we modeled both the impact of matching barcode 
a

MC3 Variants
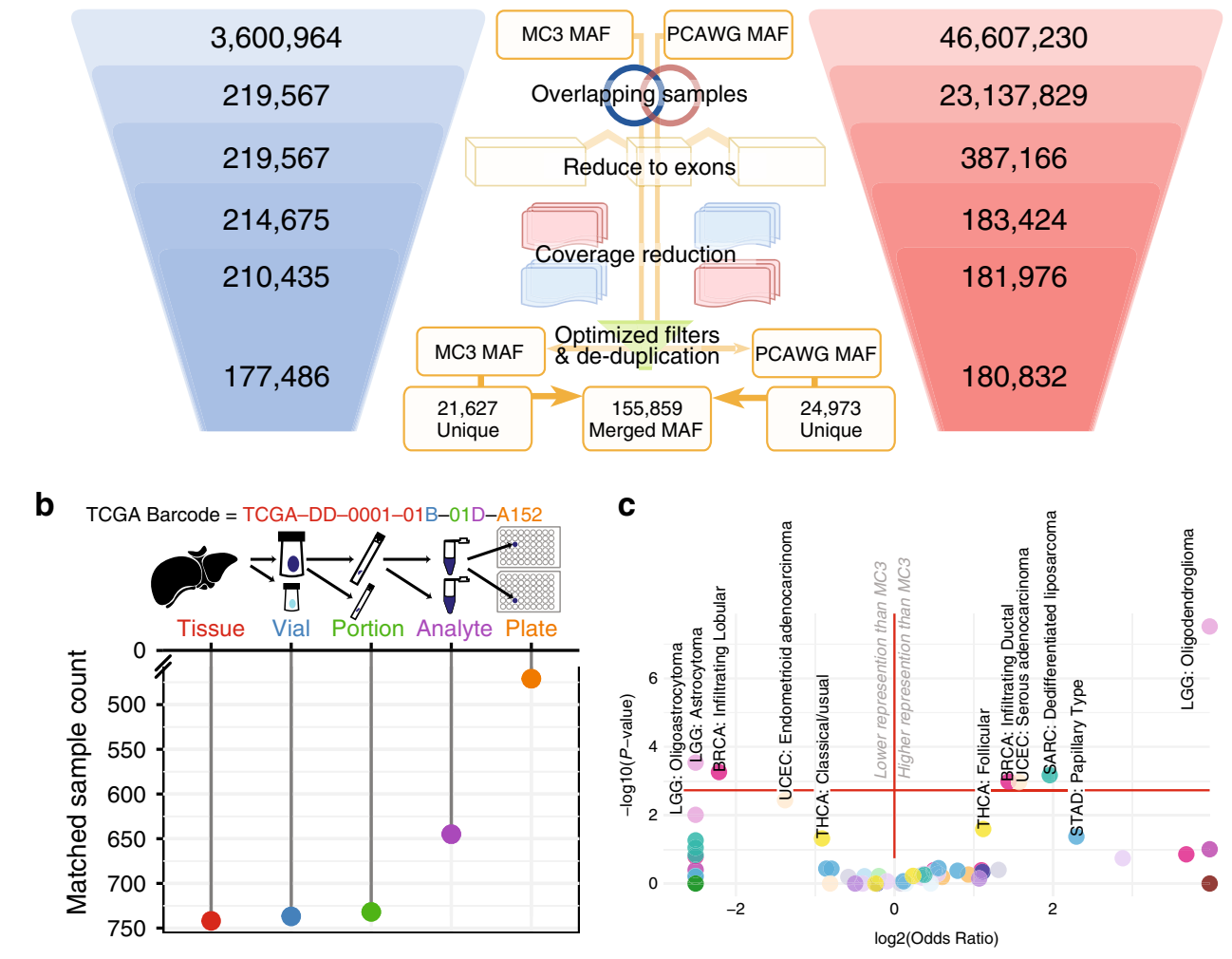

PCAWG Variants

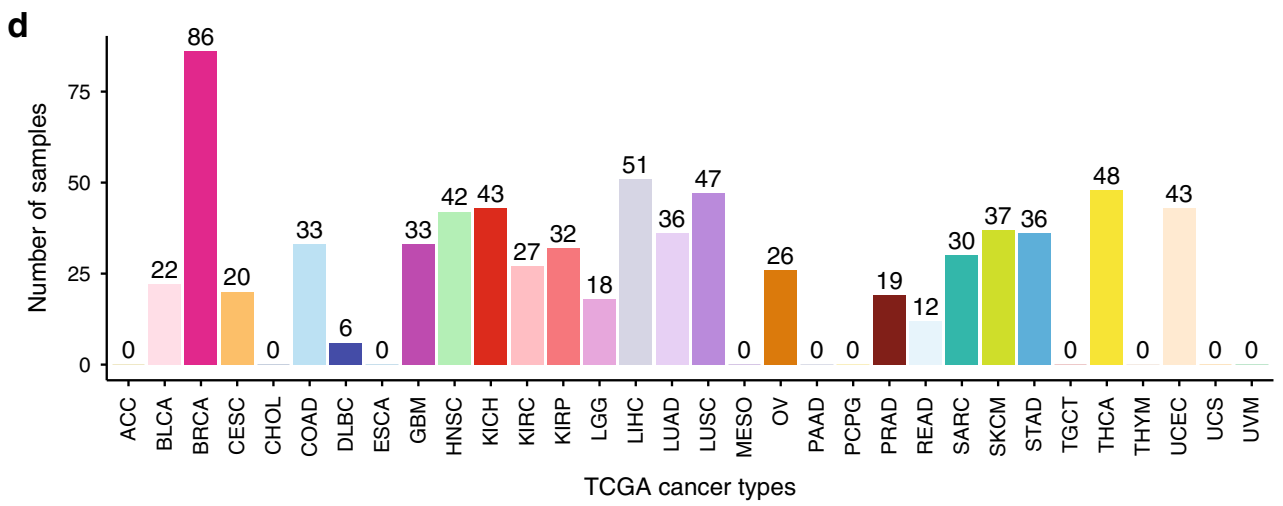

Fig. 1 Workflow and sample inclusion statistics. a A workflow diagram illustrates the number of mutations present during each step (gradient) of the filtering processes for MC3 (left, blue) and PCAWG (right, red). A brief description of each step of the intersection process is shown in between. $\mathbf{b}$ TCGA barcodes and aliquot IDs were used to match somatic sequencing. The exact match of these IDs is shown for various collection aliquots from tissue to plate. c A volcano plot highlights cancer subtype discrepancy between each PCAWG and MC3 with - $\log 10$ ( $p$-value) on the $y$-axis and log2(odds ratio) on the $x$-axis (Fisher's exact test). The horizontal red bar indicates a significant threshold after multiple testing correction. Positive values indicate an overrepresentation of a cancer subtype in PCAWG, while negative values indicate an under-representation of a cancer subtype in PCAWG compared to MC3separated by a vertical red bar. d Sample counts for each cancer type are shown in a bar chart. The colors coordinate with panel $\mathbf{c}$.

identifiers between MC3 and PCAWG and variant concordance, finding that differing barcodes did not have an appreciable impact. This result was seen for all samples, even when excluding the hypermutator (Fig. 1). Second, each AWG was able to independently select samples for WGS, which, while not affecting mutation calling, does raise potential biases when comparing PCAWG results to TCGA exome cohort data. An enrichment analysis was performed to identify which tumor subtypes may have been preferentially selected for different cancer types. We found that four tumor subtypes were enriched in the PCAWG effort from TCGA samples: infiltrating ductal breast cancer, endometrial serous adenocarcinoma, differentiated liposarcoma, and low grade oligodendroglioma $(\mathrm{FDR}<0.05$, Fig. 1c,
Supplementary Data 3, and see "Methods" section). Final tumor sample counts for each cancer type are shown in Fig. 1d.

Landscape of mutational overlap between WGS and WES calls. Limiting our analysis to coding regions with sufficient coverage yielded a total of 202,459 variants (155,859 matched, 21,627 unique MC3 variants, and 24,973 unique PCAWG mutations), with $76.7 \%$ in concordance between MC3 and PCAWG and $10.7 \%$ and $12.3 \%$ being unique in MC3 and PCAWG, respectively (Fig. 2a). Concordance can be further separated into SNPs and indels, with $79 \%$ and $57 \%$ overlapping, respectively (Supplementary Fig. 4). Variant overlap was further investigated to reveal 
a

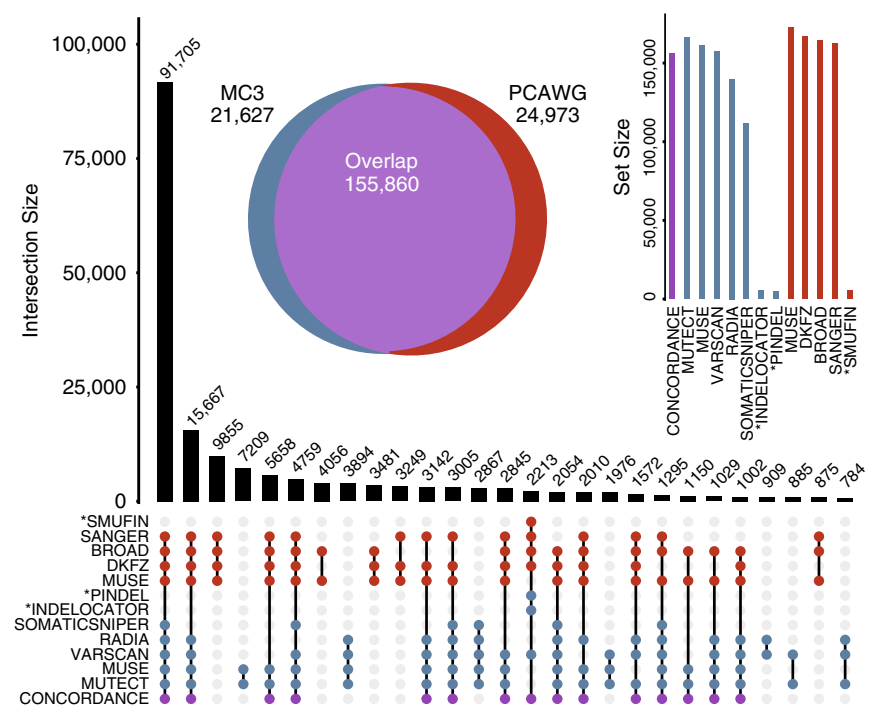

b

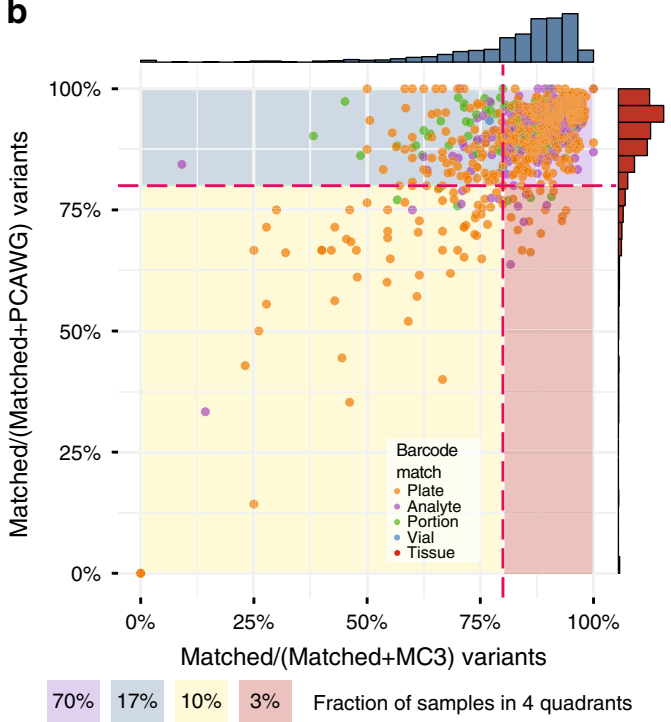

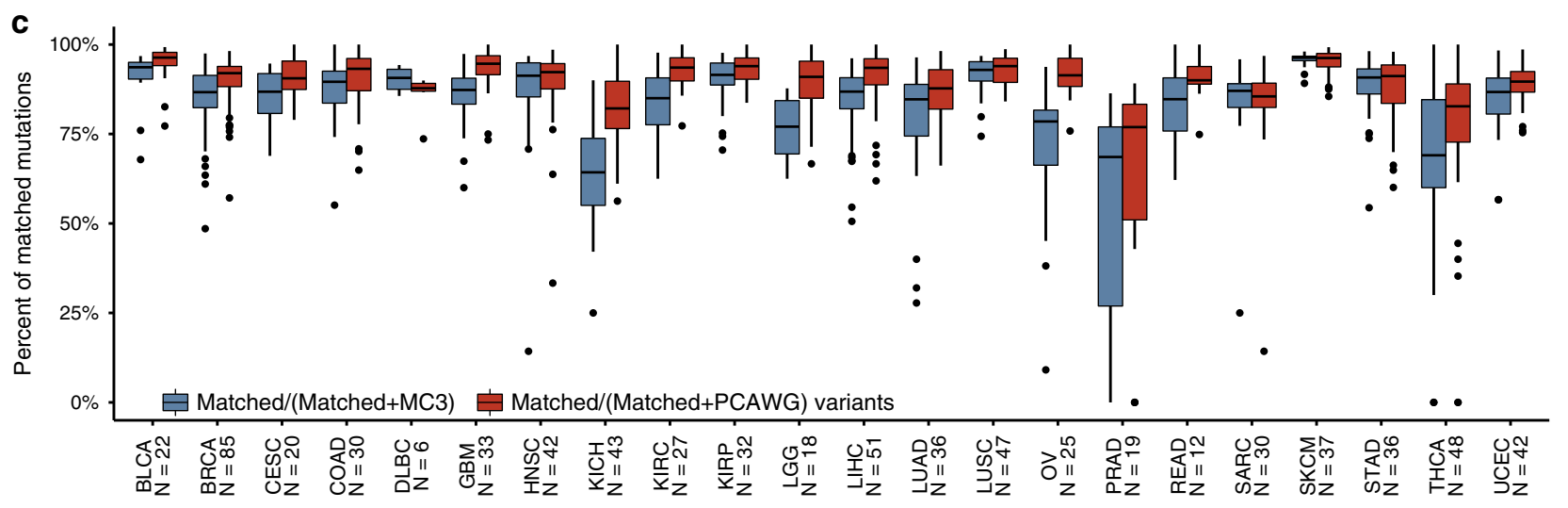

Fig. $\mathbf{2}$ Landscape of mutations overlap by caller, sample and cancer type. a UpSetR ${ }^{41}$ plot shows the variant calling set intersection by caller. The $y$-axis indicates set intersection size and the $x$-axis uses a connected dot plot to indicate which sets are considered. Only the largest 27 intersecting sets are shown. Two insets of the UpSetR plot highlight a classic Euler diagram (left), which indicates the total number of overlapping mutations. A set-size bar chart (right) illustrates the total number of mutations considered from each caller. The concordance set indicates the agreement between WES and WGS. Indel callers are indicated with an asterisk. b A scatter plot shows the amount of concordance by sample by calculating the fraction of matched variants divided by the total number of mutations made by MC3 exome sequencing and PCAWG whole genome sequencing ( $x$ and $y$-axis, respectively) below the total fraction of samples within each quadrant. Each point within the plot is related to tumor portion data collected from the TCGA barcode ID. $\mathbf{c}$ As shown above, this box plot separates panel b by cancer types (blue considers all MC3 variants, and red boxes indicate all PCAWG variants). Sample sizes are displayed for each cancer; points indicate samples that extend past 1.5 times the interquartile range; and horizontal bars within each box and whisker indicates median matched mutation fraction.

its association with mutation caller, sample, and cancer type (Fig. 2a-c). Consensus variant calling showed 91,705 (45.3\%) concordant variants were captured in the intersection of Sanger, MuSE, DKFZ, and Broad callers from PCAWG, as well as Varscan, SomaticSniper, Radia, MuTect, and MuSE callers from MC3. Notably, an additional $7.7 \%$ were identified by all SNV mutation detection algorithms, except SomaticSniper. The reduced sensitivity of SomaticSniper is related to its algorithmic consideration of tumor contamination in the matched normal (e.g., skin) for liquid tumors ${ }^{8}$. After optimizing for filtering strategies, we performed a sample level comparison and found that $70 \%$ of samples had greater than $80 \%$ mutation concordance across the two cohorts. An additional 20\% of samples had greater than $80 \%$ mutation recoverability in one or the other technique (Fig. 2b). Skin Cutaneous Melanoma performed the best among all cancer types and had the highest variant-matching rates for both MC3 and PCAWG (Fig. 2c). Generally, when considering all MC3 and all PCAWG mutations separately, we observed that
PCAWG variant matching rates were generally higher, especially for Kidney Chromophobe (KICH), Brain Lower Grade Glioma (LGG), Ovarian Serous Cystadenocarcinoma (OV), Rectum Adenocarcinoma (READ), and Thyroid Carcinoma (THCA). The differences in OV are likely driven by whole genome amplified library preparation. Generally, the median fractions for matching MC3 variants were lower than those of matching PCAWG variants. This result was unexpected because MC3 provided fewer unique variants overall, suggesting that a large fraction of PCAWG unique variants reside in a few samples. Furthermore, after accounting for hypermutators, we identified a correlation between non-silent mutations per megabase and mean consensus percentages at the cancer level in both PCAWG consensus percentages $\left(\right.$ Mann-Whitney $p$-value $\left.=1.97 \times 10^{-3}\right)$ and MC3 consensus percentages (Mann-Whitney $p$-value $=6.59 \times 10^{-4}$, see "Methods" section, Supplementary Fig. 1c, d). Despite strong rank statistics, neither set exhibited strong correlation values for MC3 variants or PCAWG variants, $R^{2}$ statistics $=0.31$ and 0.17 
respectively with the majority of cancer types exceeding $80 \%$ mean concordance. Thus, one may expect to observe slightly higher variant fidelity in samples with more mutations.

Variant allele fraction affects call-rates. After achieving a comparable data set and merging MC3 and PCAWG variants, we found that low VAF is the prevailing attribute of unique mutations. VAF is a fundamental factor in somatic variant detection, as well as sub-clonal structure prediction, and is used to predict subclonal tumor growth rates and metastatic potential. To explore the contribution of VAF, we sought to distinguish the contribution of subclonal structure and statistical chance when exploring private mutations in a single call set. We articulate our findings in six broad categories: modeling sequence noise, departure from idealized behavior, sub-clonal modeling, annotation differences, variant-caller effects, and analysis correlations.

Association of variant allele fraction with recoverability. We have observed that variants with low VAF are less likely to be reported in both call-sets. This finding relates to the lower sensitivity of somatic variant callers for variants with low VAF. To illustrate this principle, we estimated the expected overlap rate between MC3 and PCAWG at different VAFs. The sensitivity of MuSE across a range of VAFs and read depths in synthetic data was reported in Fan et al., 2016 3 . We used these reported benchmarking characteristics of MUSE to estimate the expected overlap rate between the MuSE call-sets of MC3 and PCAWG across a range of VAFs (see "Methods" section). These expectations, which involve lower overlap rates at lower VAFs, generally tracked observed data but tended to overestimate observed overlap rates, especially for predicting the recovery fraction of MC3 variants in PCAWG. (Fig. 3a) The discrepancies between expectations and observations may relate to simplifying assumptions that made this modeling possible (see "Methods" section).

More generally, we observed that VAF had a greater association with variant recovery rates than predicted by the binomial model (Fig. 3b). A random forest regression model trained on five statistics characteristics of VAF distribution per PCAWG sample and another five for that of the corresponding MC3 call-set predicted the fraction of variants per sample unique to PCAWG with 0.85 (0.86-when restricting to variants called by MUSE) Spearman correlation of test-set observations and a $0.68(0.78)$ coefficient of determination $\left(\mathrm{R}^{2}\right)$.

The strong association of VAF with recovery rates by call-set, despite modest explanatory power of the binomial, indicates important departures from idealized behavior. These departures could include explanations such as: PCR amplification violates the assumption of independence of reads, imputed read depths are systematically inflated, or some low-VAF variants represent sequencing artifacts. We conclude that non-ideal effects of VAF predict the majority of sample-level variance in fraction of cocalled variants.

Exploring subclonality. One possible explanation for some variants being private to one call-set is that the sequencing aliquots for the two sequencing projects came from subclonally-distinct microregions of the same tumor. To investigate this possibility, we tested whether the MC3 and PCAWG call-sets differed from each other systematically at the subclonal level (Fig. 3c, d). We hypothesized that tumors with a more complex subclonal structure (i.e., greater number of subclones) would have larger systematic differences in the VAF of shared variants between the MC3 and PCAWG call-sets. We found a small but highly significant effect: each additional subclone increased the average absolute difference in VAF of the shared variants between MC3 and PCAWG by 0.003 , with a $p$-value of $1.3 \times 10^{-11}$ (linear regression); this effect reversed after controlling for tumor purity, indicating that the observed trend does not provide evidence of this interesting concept in re-sequencing (see "Methods" section for details). We do not have evidence that systematic VAF differences between call-sets of the same underlying sample associate with tumor heterogeneity. Real time effects of VAF differences between these two data sets can be observed using the online MAFit tool (Fig. 4).

Annotation differs by call-set. Genome annotation is critical for biological interpretation and downstream analysis of sequencing data. In order to avoid issues that arise from annotation differences, we only considered genomic locations in our intersection strategy. In doing so, we observed 2153 annotation differences where MC3 and PCAWG had different genes annotated for the same mutation. After restricting the mutation type to missense mutations and indels, 789 annotations differences remained. Most of these had the same mutation types annotated by both call-sets (690 SNPs, 15 insertions, 50 deletions), but some discrepancies remained. Notably, 413 out of 789 mismatch variants are labeled coding in MC3 but non-coding in PCAWG (Supplementary Data 4). We also observed four mutations that were annotated as cancer gene mutations by MC3, but as non-cancer gene mutations by PCAWG, and another four mutations that were annotated as cancer gene mutations by PCAWG, but as non-cancer gene mutations by MC3. One such example subsumed two mutations on chromosomal location 3p21.1 (genomic locations chr3:52442525 and chr3:52442604) that were annotated as missense mutations of BAP1 by MC3, but as 5'Flank SNPs of PHF7 by PCAWG. While identical pipelines resolve such differences, we stress the potential for misinterpretations when combining these publicly-available datasets.

Effects of software. Another important issue we assess is the degree to which differences in bioinformatics pipelines impact concordance. We extracted calls from MuSE and MuTect, both of which were executed on each dataset, and examined 6 subsets of results: MuSE-only-calls and all calls save MuSE-calls (the complement), MuTect-only-calls and their complement, and MuSE + MuTect calls and their complement. MuSE and Mutect each generate around $95 \%$ of the total calls, of which each respective subset shows close to $80 \%$ concordance between WES and WGS (Supplementary Fig. 5). These call sets themselves overlap almost completely, with their combination (MuSE + MuTect) giving a marginally higher concordance. Conversely, the data-specific caller combinations (referred to above as the complements) each furnish small call sets which vary considerably between WES and WGS (concordance as low as 15\%). Because of the vast difference in the sizes of the MuSE/MuTect and the complementary call sets, there is little difference in the original analysis versus analyses restricted to variant callers common to both platforms. Differences in software pipelines do not appear to be significant confounding factors in concordance here.

Effects on higher-level analysis. We also sought to assess how higher-level analyses might be impacted using mutation signature analysis as a representative. We ran SignatureAnalyzer28 to ascertain signatures between matched WGS and WES samples for each case. A total of 563 of 739 cases (76\%) showed the same dominant signature between WES and WGS and the multielement signature vectors for each case are very highly correlated with one another, the average Pearson coefficient being almost $90 \%$, with a cohort significance of $<2 \times 10^{-6}$ (Fisher's Test, 
a

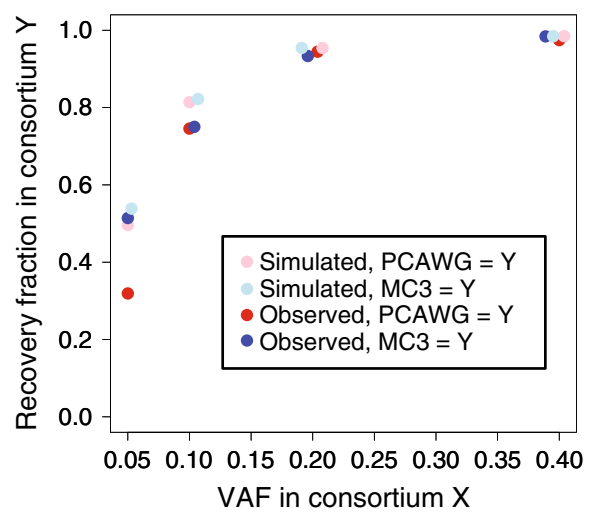

C

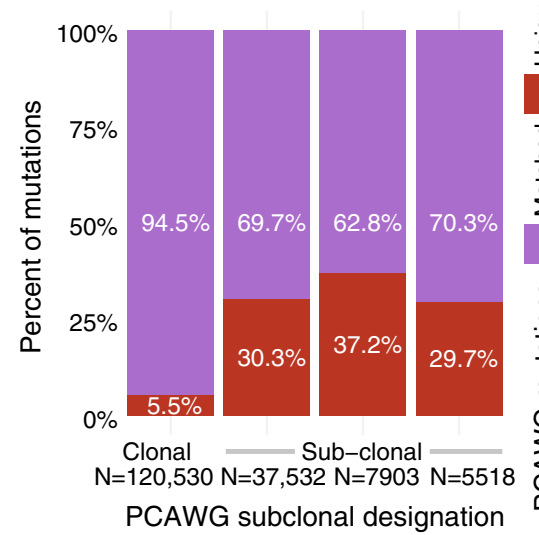

b

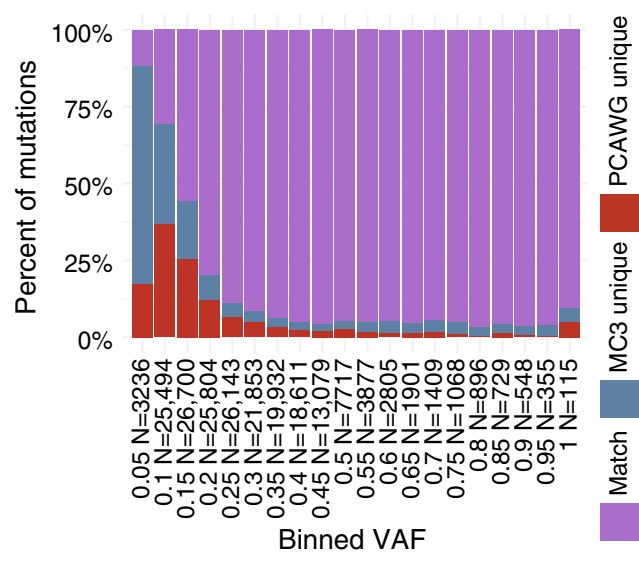

d

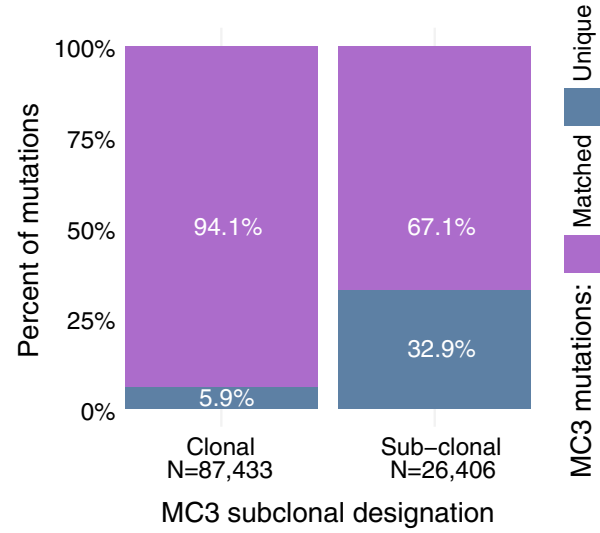

Fig. 3 Recoverability simulation and effects of subclones on mutation concordance. a Observed recovery rate of PCAWG variants in MC3 (red) and of MC3 variants in PCAWG (blue), alongside sequencing noise simulations calculated from random draws of a binomial model that incorporates the VAF and estimated read depth at each site (light red simulates PCAWG recoverability of MC3 variants, and light blue simulated MC3 recoverability of PCAWG variants). $Y$-axis is described with legend. $X$-axis displays $V A F$ of the comparative data set in regard to $Y$. $\mathbf{b} A$ stacked bar chart displays the proportion of matched and unique variants ( $y$-axis) for different VAF bins ( $x$-axis). 180 variants did not provide read count information and were removed from this figure. c Stacked proportional histogram shows the fractions of PCAWG matched mutations (purple) and PCAWG-unique mutations (red). Mutations were restricted to SNVs, and subclonality predictions are indicated as either 'Clonal' or 'Sub-clonal'. Columns 2-4 reflect sub-clonal assignment provided by PCAWG (Note: only a few samples reported five predicted subclones and were not included in this analysis). The number of variants represented for each clonal assignment is shown on the $x$-axis. $\mathbf{d}$ Similar to panel $\mathbf{c}$, a stacked proportional histogram illustrates the proportion of matched and unique variants for MC3 which provide estimates of total number of matched or unique variants called by MC3.

"Methods" section, Supplementary Fig. 6). These observations suggest that signature analysis is relatively insensitive to data type when concordance is high, as it is here.

Landscape of private WES and WGS mutations. After identifying many possible sources of variation among private variants, we sought to characterize the fraction of variation explained by previously identified factors (Supplementary Fig. 7, see "Methods" section). As displayed, subclonal and low VAF variants make up the largest fractions of explained variants for private MC3 and PCAWG variants. Notably, for private MC3 calls, indels (not called by MuSE or MuTect) are the next highest source of variation explained. GC-content and poor performing cancers such as THCA, KICH, and PRAD make up a smaller portion of the total number of private mutations.

Variants present in only one public call-set. We sought to classify cancer driver mutations uniquely identified by MC3. After removing two outlier samples having excesses of unique mutations (TCGA-CA-6717-01A-11D-1835-10, TCGA-BR-6452-
01A-12D-1800-08), we observed 424 mutations in cancer genes ${ }^{28}$ (median read depth $=97$, median alternative allele count $=9$ ) The four most frequently mutated genes were: KMT2C (22mutations), PIK3CA (12), SPTA1 (9), and NCOR1 (9). Interestingly, the majority of unique PIK3CA mutations not identified by PCAWG were at 2 locations: E542K/G (5), and E545K (4). Whether this phenomenon reflects technical bias of WGS or is a product of subclonality warrants further investigation.

The MC3 effort produced two mutation files: one controlled access somatic mutation file that represents nearly all mutations found by all callers, and a second was modified by the scientific community for public use. There are two critical differences in these files involving the reporting of mutations in exonic regions and mutations reported by a single variant caller. Since we limited our analysis strictly to exonic regions, we observed that $92 \%$ of the 9138 PCAWG private mutations found in the MC3 controlled access file were only identified by a single variant caller (Supplementary Fig. 8). As expected, the highest unique variant caller overlap was observed in MuTect and MuSE, two tools that were used by both MC3 and PCAWG. This observation accounts for $30 \%$ of PCAWG private variants. 
a

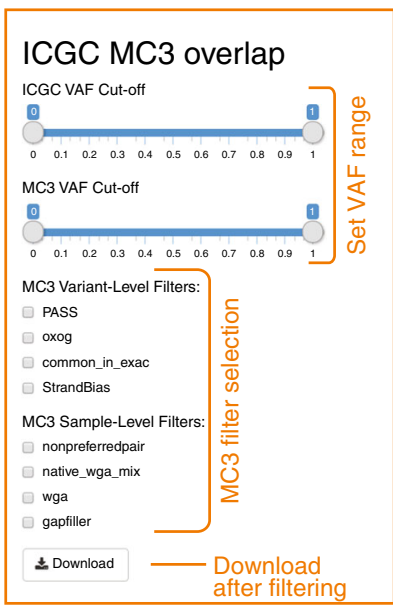

b

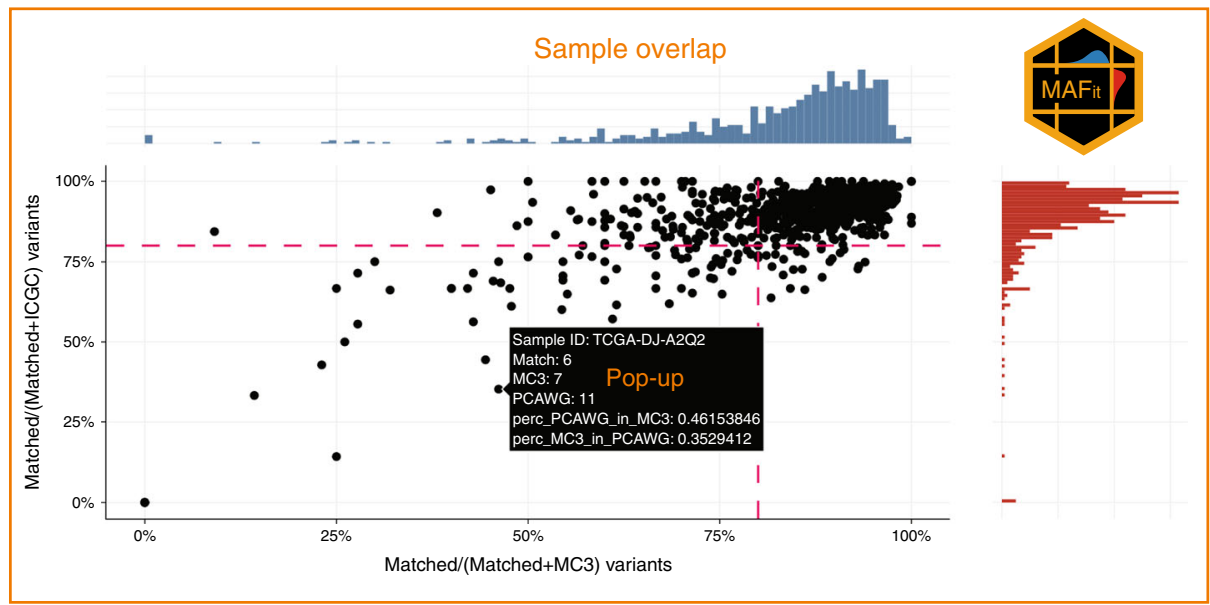

C

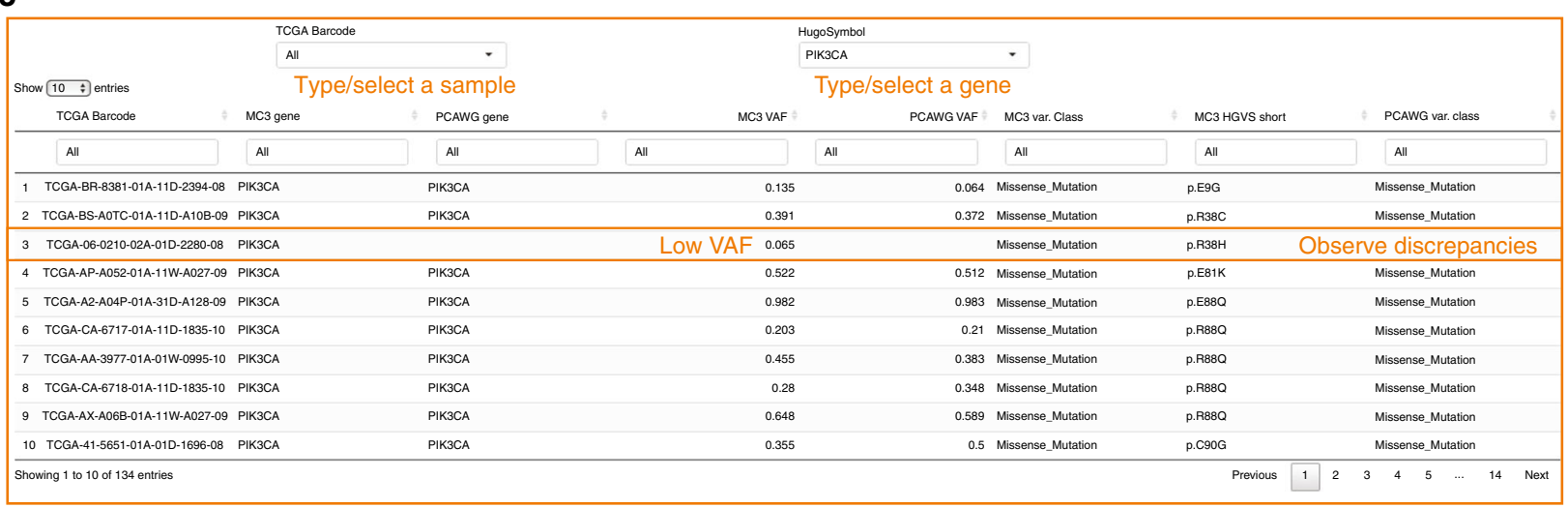

Fig. 4 Screenshots of online tool MAFit. Here we display screenshots from the MAFit on-line interface. Currently there are three main components to the interface: a A side panel shows sliders and radio buttons to filter data set to remain inclusive. In addition, a download button is available that will download the underlying data table. $\mathbf{b}$ MAFit rebuilds Fig. $2 \mathrm{~b}$ in the first tab of the on-line interface. Each alteration to the radio buttons or VAF sliders will result in an updated figure. In addition, if one's hovers over a point on the scatter plot, a pop-up window will automatically display, providing the user with basic statistics used to calculate that point, i.e., total number of mutations, number of unique and matched mutations. $\mathbf{c} A$ table is also presented based on the selection criteria in panel $\mathbf{a}$.

We investigated how many variants unique to the MC3 somatic public access call-set could be found in the PCAWG germline call-set for the same patients. We identified a total of six such variants (each in a different sample), five of which were flagged in the MC3 public call-set with one filter or another. Overall, this indicates that variants that have been incorrectly designated as germline or somatic are an extremely uncommon source of variation between the two projects.

Variants in GC-extreme intervals. Since it is well-known that the efficiency of exome capture is adversely affected by very high or very low GC-content ${ }^{29,30}$, we sought to test whether GC-content was associated with call rates in MC3 and PCAWG. We used a plug-in for VEP ${ }^{31}$ to annotate all matched and private SNVs with $\mathrm{CADD}^{32}$ in order to annotate each variant with the percentage of the neighboring 100 bases that are a G or C. First, we assessed how the distribution of read depth across GC-content changes between MC3 and PCAWG (Fig. 5b). PCAWG was found to have a fairly uniform read depth across GC-content bins, while MC3 read depth was concentrated in regions of moderate GC-content (Fig. 5c). The low read depth in MC3 at regions of extreme GCcontent was in turn associated with lower variant recovery rates in these regions but did not grossly affect the number of variants recovered by $\mathrm{MC} 3$ because regions of extreme GC-content are relatively rare in the genome overall and in exome-capture regions in particular.

An in-depth analysis of these regions revealed that 76 mutations in known driver genes, identified in the combined TCGA data by Bailey et al. 2018, were missed in GC poor (GC fraction $<0.3)$ or GC rich $(\mathrm{GC}>0.7)$ regions $^{28}$. Three such instances revealed $V H L$ mutations in KIRC that were overlooked in GC rich regions of this gene (two of these three recur). In addition, these 3 samples are not reported to carry a VHL mutation in the MC3 public data set. Other such instances include 7 SOX17 mutations, LATS2 (6), and CACNA1A (6). These findings emphasize the advantages of uniform coverage using WGS.

The bases flanking a mutation (tri-nucleotide context) affect mutation rate, which should be approximately equal between MC3 and PCAWG, and also the rate of introduction of sequencing artifacts. Large differences in the call-rates of MC3 and PCAWG and particular nucleotide sequences could indicate a sequencing artifact unique to one or the other call-set, which might arise from different procedures for computationally filtering or biochemically preventing sequencing oxidation products. Therefore, we sought to test whether the trinucleotide context of variants correlated with relative call-rates in MC3 and 
a

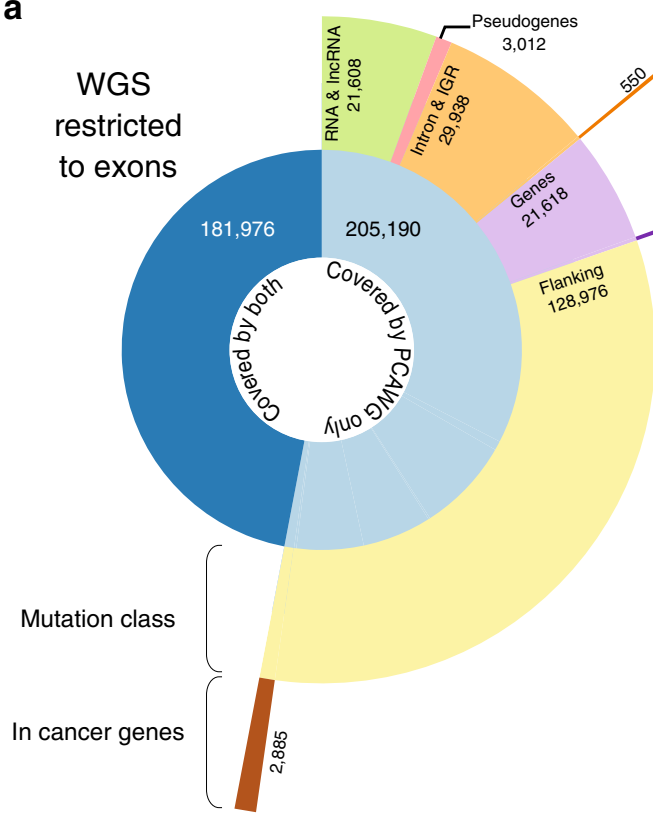

b

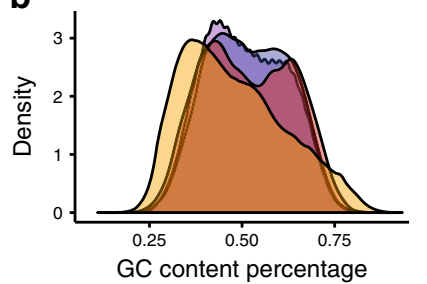

d

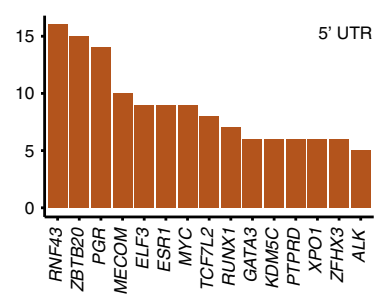

e

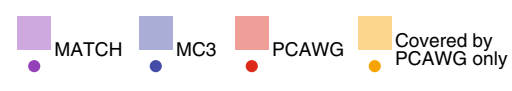

C

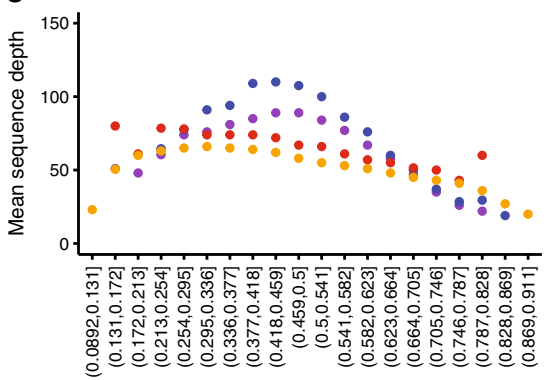

Binned GC content percentage
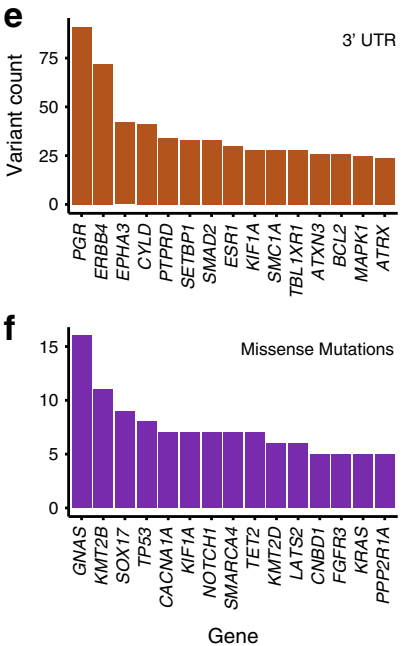

g \begin{tabular}{|l|l|l|l|l|l|l|l|l|l|l|l|l|l|l|}
\hline ACVR2A & ARID5B & ERBB4 & KIF1A & MGA & NIPBL & PDS5B & PIK3R1 & PTPRD & RET & STAG2 & USP9X & ZFHX3 \\
\hline
\end{tabular}
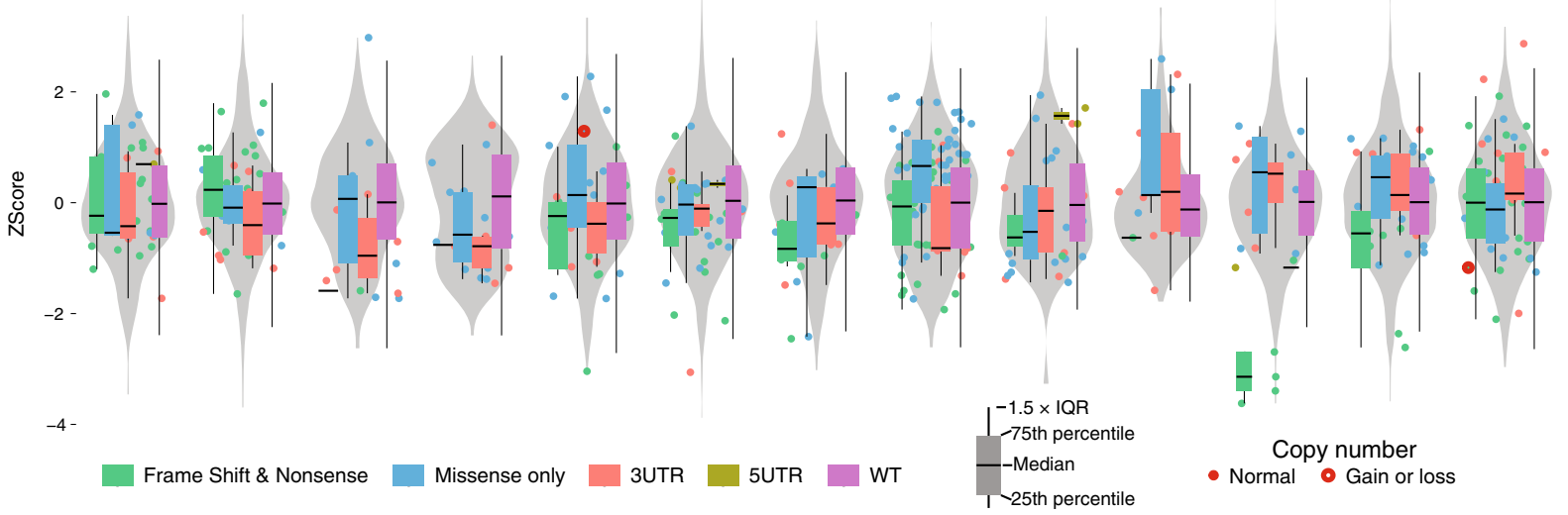

- Normal $\bullet$ Gain or loss

Fig. 5 WGS mutations in exonic regions not captured by WES. a A sunburst diagram provides a breakdown of variants that are removed during the coverage step of the tool. The innermost circle represents the total number of variants identified upon filtering for exome beds used by MC3. Then, we restrict PCAWG variants to well-covered MC3 regions for each sample. The majority of gencode.v19 annotated and the BROAD target bed file of exonic regions are sufficiently covered by PCAWG in flanking regions: $3^{\prime} U T R s, 5^{\prime} U T R$, and 5'Flanking. The outermost ring illustrates the number mutations identified by PCAWG that were poorly covered by MC3. b A density plot illustrates the density of percent GC-content from a 100 bp window surrounding a variant. Four variant-sets are displayed: matched, private to $M C 3$, private to PCAWG, and we extend our dataset to include exonic variants not covered by WES but sufficiently covered in WGS (Covered by PCAWG only). c A scatter plot displays mean sequence depth ( $y$-axis) by increasing GC-content bins $(x$-axis). Points are colored according to variant set (same as panel $\mathbf{b})$. $\mathbf{d}$-f Total annotated mutations counts from 3 different annotated regions are shown for 5UTR, 3UTR, and missense mutations, respectively. $\mathbf{g}$ Expression Z-Scores for 3'UTR using all TCGA-UCEC samples. Cis-RNAseq expression violin plots are displayed for 13 genes. On top of the gene-level distribution violin plot, box and whisker plots display sample expression based on mutation classification (box include 25th quantile to 75 th quantiles, and whiskers extend to 1.5 times the interquartile range).

PCAWG. Before applying the MC3 OxoG filters, we found a huge predominance of $\mathrm{CA}$ variants unique to $\mathrm{MC}$, with the trinucleotide contexts most specific to one database or another being 7-9 times more specific than the least specific trinucleotide contexts. After applying the MC3 OxoG filters, nucleotide contexts differed by less than four-fold in their specificities. The residual differential specificity by trinucleotide context after filtering can either indicate differences in sequencing artifact abundance and filtration by project, or could merely be a consequence of the fact that nucleotide context is also correlated with VAF and the distance from transcription start sites, which may independently affect MC3 and PCAWG relative call-rates.

We extended the nucleotide context and performed mutation spectrum analysis, comparing all MC3 and all PCAWG mutations found after restricting the two data sets to exonic regions as described above (Step 3 of Fig. 1a). We then calculated 
transition and transversion frequencies in each cancer type. After removing hypermutated samples and OxoG artifacts, we used a chi-squared test to determine the similarities and differences between cancer types in the full exome space compared versus the captured exome space. Strikingly, we did not identify significant differences in mutation spectrum in the majority of cancers. We did observe significant differences $(F D R<0.05)$ in the mutation spectrum for COAD, KICH, LUAD, and OV (Supplementary Data 5). These observations included strong discrepancies for AG and CG transition differences in $\mathrm{KICH}$ and $\mathrm{OV}$, respectively. AT and CA transversions contributed mostly to COAD and LUAD differences (Supplementary Fig. 9). While these differences may reflect sequencing artifacts, such as whole genome amplified DNA in OV or low sample size, we believe the data can still provide more information pertaining to additional cancer genes and oncogenic mechanisms.

Non-Coding/Flanking intersections with low coverage. With the growing use of WGS in many labs, we sought to identify which mutations are gained by extending to this form of data. One major observation from our pipeline highlighted that some variants in exome regions were not well covered by WES (Fig. 1a Step 3). Using this mutation set we investigated the most recurrent members as derived by WGS but not by MC3 in exonic regions as defined by gencode.v19 (Fig. 5a). We observed 697 mutations in cancer genes ${ }^{28}$ uniquely called by WGS (Supplementary Data 6). We defined flanking mutations as all nontranslated mutations near exons, i.e., 5'UTR, 3'UTR, 5'Flanking, and $3^{\prime}$ Flanking regions, as they make up the majority of mutations not present in the MC3 public MAF. Recurrent mutation analysis identified the most frequently mutated genes in 5'UTR (Fig. 5d), 3'UTR (Fig. 5e), and missense mutations (Fig. 5f). We found the most frequently mutated 3'UTR in cancer genes was PGR (91 mutations allowing for multiple mutations per sample), followed by ERBB4 (72), EPHA3 (42), CYLD (41), and PTPRD (34). To extend this analysis, we used RNAseq data collected by TCGA to determine mutation type specific cis-expression patterns, which clearly shows correlation of UTR mutations on RNA abundance (Fig. 5g).

Finally, similar to previous studies ${ }^{33,34}$, we investigated the potential effect of non-coding mutations when determining significantly mutated genes (SMG). Using $\mathrm{MuSiC}^{35}$ with the no-skip-non-coding option, we rescued non-coding mutations annotated by PCAWG and included them in the significantly mutated gene (SMG) analysis. We only performed SMG analysis on cancer types having greater than 35 samples (BRCA-BreastAdenoCa, HNSC-Head-SCC, KICH-Kidney-ChRCC, LIHCLiver-HCC, LUAD-Lung-AdenoCA, LUSC-Lung-SCC, SKCMSkin-Melanoma, STAD-Stomach-AdenoCA, THCA-Thy-AdenoCA, and UCEC-Uterus-AdenoCA). We initially identified potential driver-gene candidates (FUT9, MMP16, SNHG14, and $S F T P B$, Fig. 6) not previously reported in Pan-Cancer whole genome analysis, but further investigation did not support these candidates with the exception of SFTPB.

SFTPB (FDR 1.56e-07) in LUAD was recently reported to be significantly mutated using a larger set of these same data ${ }^{34}$. As reported, this gene is involved in a lineage-defining surfactant protein. While six mutations contributed to its SMG status, only 1 3'UTR mutation was reported for LUAD in the MC3 controlled data set. Furthermore, this single indel was only found by one variant caller (Varscan). We confirmed the impact of SFTPB UTR mutations by performing a genome-wide association analysis of expression differences and found that samples with SFTPB mutations showed lower RNA abundance in PCDHA7, a gene known to be involved in cells' self-recognition and non-selfdiscrimination (chi-squared $p$-value $3.6 \times 10^{-8}$ ). While other promising candidates exist, such as FUT9, a fucosyltransferase involved in organ bud progression during embryogenesis and has been implicated in cancer initiation ${ }^{36}$, we found no additional evidence for supporting its driver status.

\section{Discussion}

The research community is increasingly leveraging technology advances to integrate data at larger scales. We performed a comparative evaluation of $\sim 750$ samples with joint exome and whole genome sequencing mutation calls provided by two consensus mutation calling efforts, MC3 and PCAWG. This joint data set is encouraging, suggesting that $\sim 80 \%$ of the predicted somatic mutations were captured by both efforts. Furthermore, a combined $90 \%$ of samples have greater than $80 \%$ variant concordance when considering covered exonic mutations from individual cohorts separately. Analysis of this data set also revealed three major contributors to variant discrepancies: (1) low variant allele fraction, (2) variant filtering decisions, and (3) technological limitations. Software differences were not an appreciable confounder.

Distinct advantages and disadvantages accompany somatic mutation calling when utilizing captured WES or WGS. We found that $\sim 70 \%$ of the discrepancies between whole genome and whole exome sequencing are influenced by low variant allele fraction. This information holds many implications in identifying subclonal heterogeneity in the tumor of interest. Other discrepant calls originate from the decisions made on how to filter and distribute publicly available mutation calls. Higher-order mutation signature analysis does not appear to be inordinately affected by these differences. We show that reported germline variants were negligible, but nearly $30 \%$ of the private PCAWG mutations were not reported by MC3 because only a single variant detection algorithm identified them. We want to emphasize that, while somatic variant detection in cancer is commonplace, there are still many issues to reconcile.

Finally, we found additional mutations only observable in exonic regions using either WES or WGS. For example, WES uniquely identified 424 mutations in cancer genes with median VAF of $\sim 10 \%$. We also highlight $~ 700$ WGS mutations from cancer genes, of which $\sim 10 \%$ are attributable to regions of high and low CG-content; thus, highlighting the advantages of more uniform coverage from WGS.

Only about $2 \%$ of the genome is protein coding. For the last dozen years, cancer genomics has provided a comprehensive molecular characterization of many different tumor types, thanks in large part to The Cancer Genome Atlas and other publicly funded efforts. The community is just starting to explore how exomics, transcriptomics, proteomics, and methylomics can be woven together across this $2 \%$ of the genome. We anticipate a general transition from WES to WGS, but this analysis is meanwhile reassuring that few clerical mutations were overlooked in WES and that WGS is capable of recapitulating previous genomic findings.

\section{Methods}

Human research participants. The Cancer Genome Atlas (TCGA) collected both tumor and non-tumor biospecimens from human samples with informed consent under authorization of local institutional review boards (https://cancergenome.nih. gov/abouttcga/policies/informedconsent).

Sample overlap. TCGA barcodes carry metadata that reflect tumor portions and different aliquots. As noted in Fig. 1b, TCGA barcode differ slightly in the comparison between MC3 and WGS aliquots. A brief description of the breakdown of the TCGA barcode is outlined below. 
a

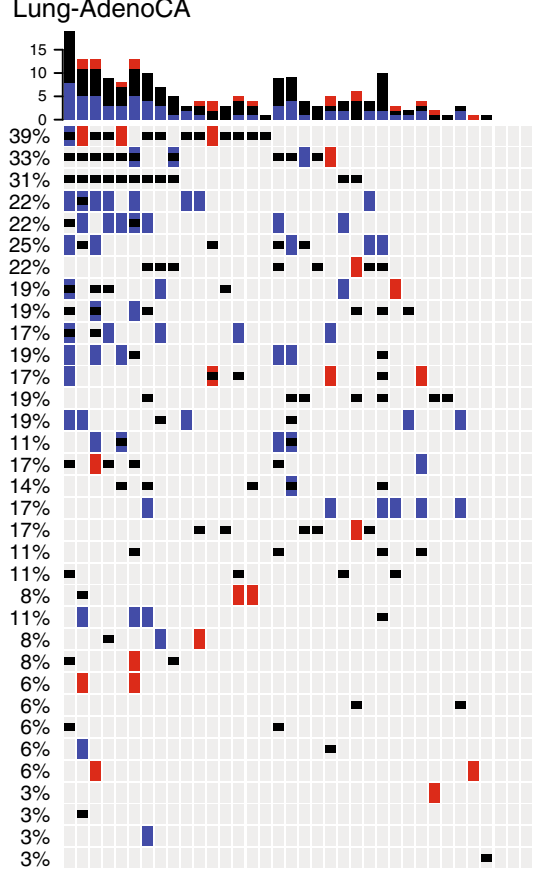

b

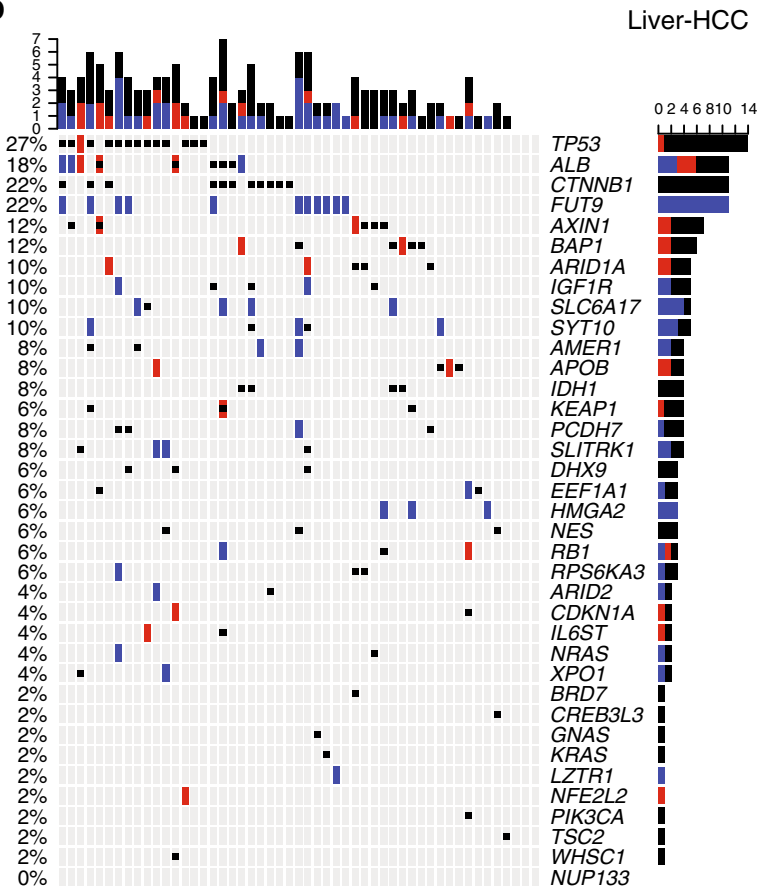

Alterations $\square$ CODING $\square$ INDELS $\square$ NONCODING

c

Lung-SCC

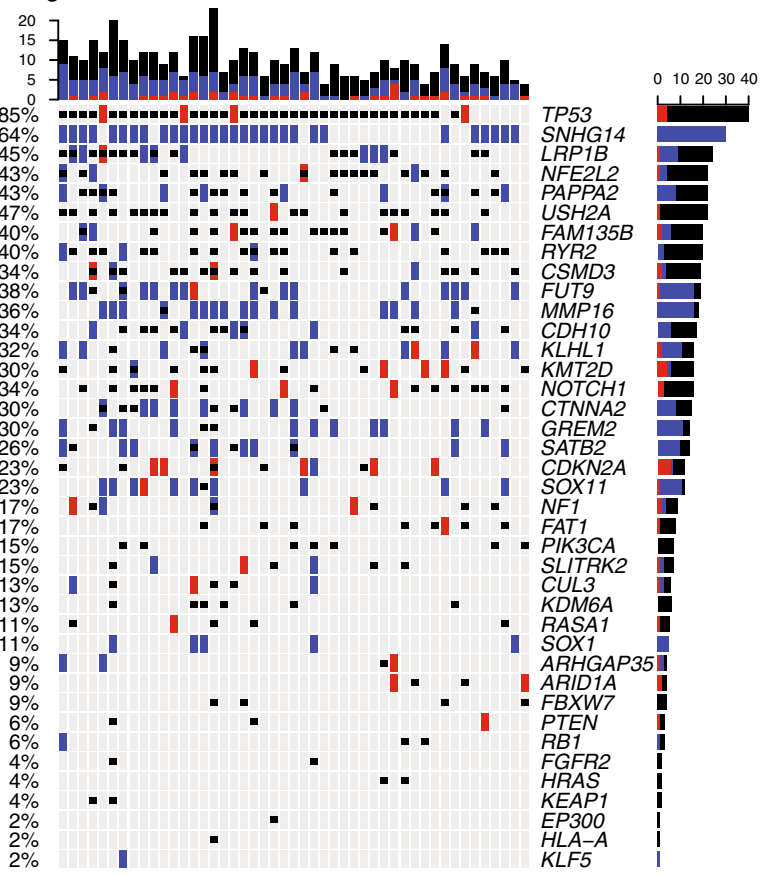

d

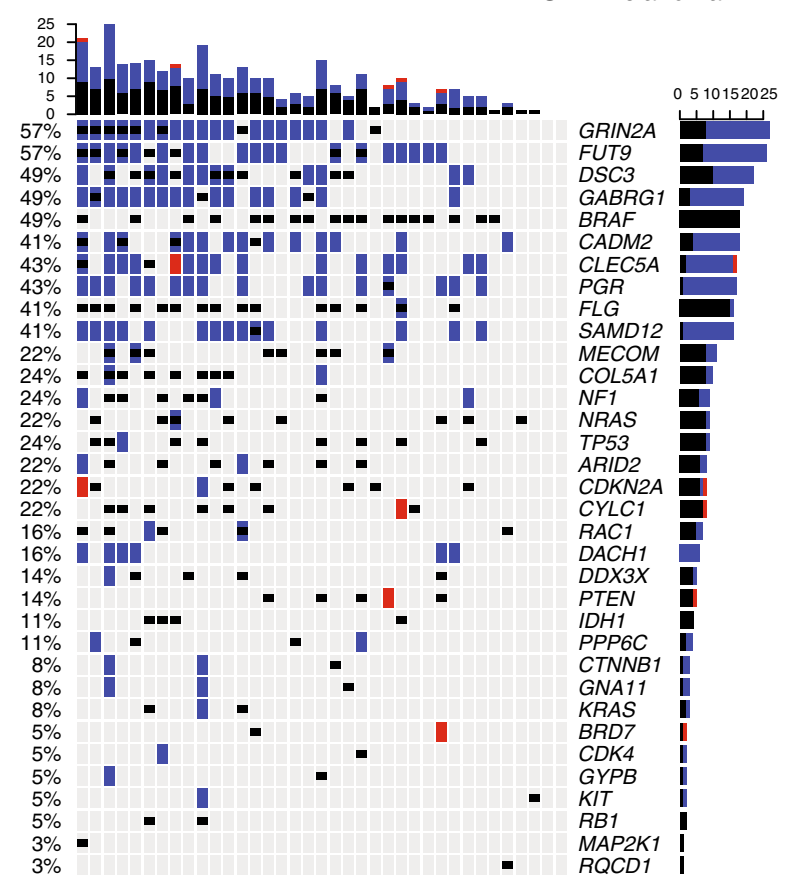

Fig. 6 Significantly mutated gene analysis with the inclusion of UTR mutations. OncoPrint plots were generated using the R package ComplexHeatmap 42 for four cancer types: LUAD (a), LIHC (b), LUSC (c), and SKCM (d). We report all SMGs identified by Bailey et al. 201828, as well as top significantly mutated gene hits from MuSiC that include non-coding mutations. While many non-coding mutations look promising, further investigation yielded little support for driver identification status.

Example: TCGA-DD-0001-01B-01D-A152

- TCGA-Project

DD-Tissue source site: the tissue location of tumor that matches clinical metadata.

0001-Participant code

01-Sample type: i.e., solid tumor (01), primary blood derived tumor (03), solid tissue normal (11), blood derived normal (10)

B-Vial: the order in a sequence of samples, i.e., $\mathrm{A}=$ first in sequence, $\mathrm{B}=$ second in sequence

01-Portion: sequential order of the $100-120 \mathrm{mg}$ of samples

D-Analyte: molecular analyte type for analysis, i.e., D for DNA and W for WGA.

- A152-Plate: sequential location of a 96-well plate 
A lookup table outlining these fields is located at the GDC: https://gdc.cancer. gov/resources-tcga-users/tcga-code-tables. In order to determine the role of aliquot differences in assessing mutation concordance, we re-analyzed the clonality and overall mutation overlap after stratifying for exact barcode differences. We observed that the effect of matching barcodes on match variant frequency has little effect.

Assessing cancer subtype selection preference. Analysis working groups for TCGA projects were primarily subdivided according to cancer types. Scientific experts gathered in consortia from around the country to participate in characterizing many tumors using high throughput data generated on many substrates, e.g., WES, RNAseq, etc. At the conclusion of these projects, groups were asked to hand-select a subset of samples to perform validation sequencing (WGS, the samples used in this analysis). The selection criteria differed from group-to-group and sometimes resulted in an overabundance of one subtype over another. To determine cancer subtype selection bias, we performed an enrichment analysis. Using clinical data we calculated (for each cancer type) the subtype fraction in the WES cancer cohort and measured whether the fraction was similar to WGS set of samples using a Fisher's exact test.

Defining exonic regions. We used the same definition as Ellrott et al. to reduce whole genome and exome calls to define genomic coordinates ${ }^{27}$.

Coverage calculations. Fixed-step (step $=1$ ) Wiggle coverage files (.wig) for both MC3 and PCAWG were provided by the Broad Institute. The wig format is a binary readout of sufficient sequencing coverage for genomic data. Here, sufficient coverage is defined as bases with 8 or more reads at a given location. These wig files were processed and reduced to exonic regions using the wig2bed function from BEDOPTS ${ }^{37}$.

After the preliminary screen of coverage-reduced MAF files, we observed that matching mutations (identified by PCAWG and MC3) were removed from one technology and not the other after the coverage reduction step. To account for this issue, we performed a self-coverage reduction step to that identified 6460 mutations. We describe some properties of those mutations here. The median tumor depth reported by MC3 from these variants is 12 reads $(+/-3$ median absolute difference). The median tumor depth reported by PCAWG in this region is 39 reads $(+/-35.6$ median absolute difference), suggesting wide variance of tumor read depths that were removed. However, the mode tumor depth of the PCAWG variants was 13, justifying this removal of variants with low read support. Finally, we determined how many of these poorly-covered variants originated from cancer driver genes. We observed 126 mutations from the MC3 file, and 156 cancer mutations were eliminated at this stage in the comparison.

Overlapping mutations. After reducing the variants to be within exome sequencing target region, within same exon definitions, and having enough sequencing depth, the remaining variants from ICGC and PCAWG were stored in a SQLite database to enable fast lookup. We then executed a full join between the two sources of variants by matching the donor ID, sample ID, and the genomic range of each variant. The full join output was further cleaned up to remove duplicated filters due to naming variations and duplicated variants due to DNPs.
- Matching IDs
Matching chromosomes
End position greater than or equal to start position
Start position is less than or equal to end position.

Deduplication of variants. After merging the PCAWG and MC3 data, we observed different strategies were taken by MC3 and PCAWG to capture neighboring variants, i.e., complex indels, di-nucleotide (DNP) and tri-nucleotide (TNP) polymorphisms. To address complex indel events (SNVs in indel regions), the MC3 working group absorbed the variants made by SNV callers into the assignment made by Pindel. Conversely, PCAWG merged DNP and TNP events into a single event. These strategies resulted in many duplication events from MC3 and PCAWG: 1731 and 62, respectively. These events encompassed 3457 and 119 events, respectively. To address these differences, we merged PCAWG variants into MC3s complex indel events, and MC3 variants into single DNP or TNP events.

Filtering optimization. After reducing the starting pool of possible mutations from 746 samples to covered exons, we sought to identify the optimal set of MC3 filters that provide the largest number of samples with greater than $80 \%$ concordance from the two technologies with the simplest schema. This was performed comprehensively using all possible combinations of filters, often with more than one filter per variant, with the MC3 cohort (131,071 filter combinations). Filter flags include: "common_in_exac", "gapfiller", "native_wga_mix", "nonpreferredpair", "oxog", "StrandBias", and "wga". We pre-defined the exclusion of variants in MC3 flagged as OxoG along with the inclusion of all PASS variants. The comprehensive filter analysis resulted in two major clusters of variant recoverability (Supplementary Fig. 3). Here, we observed the computational trade-off of identifying more matched variants at the cost of more unique MC3 calls. Below, we highlight five strategies considered for analysis (Supplementary Data 2).

1. Only consider variants labeled PASS by the MC3 filter column.

2. Only remove variants labeled OxoG by MC3.

3. Prioritize G1 (samples in the most recoverable quadrant, MC3 and PCAWG samples with greater than or equal to $80 \%$ from both efforts.)

4. Prioritize total number of matched variants.

5. Maximize total number of samples in the most recoverable quadrant (Fig. 2b) while maximizing the difference between unique MC3 variants and matched variants thus generating fewer unique calls.

After considering complexity, we chose to move forward with strategy 2 for the entirety of this study due to its simplicity and relative similarity to other filtering schemes. We recognize that by selecting a single filtering strategy, we are limiting the data slightly and likely introducing some false positive variant calls. However, this strategy allowed us to maintain larger sample sizes and to capture $\sim 15,000$ more matched variants than the PASS only strategy at the cost of $\sim 3500$ unique mutations calls for MC3.

Assessing mutations per megabase and cancer type concordance. Mutations per megabase data were collected from the broader TCGA dataset and reduced following the same methods outlined previously ${ }^{28}$. Briefly, this systematically removed hypermutators from the dataset. This resulted in a set of 625 samples from the MC3/PCAWG dataset studied here and 8852 TCGA samples. Both Pearson and Mann-Whitney correlations statistics were performed to assess the association of non-silent mutations per megabase and concordance statistics.

Simulation of sequencing noise and recoverability. Fan et al. benchmarked the sensitivity of MuSE at recovering somatic variants across 24 combinations of VAF and read depth ${ }^{3}$. When simulating the recovery of PCAWG variants in MC3 we assumed that the VAF observed in PCAWG was the true VAF. We matched the observed VAF of each variant to the closest VAF reported in Fan et al.

For our analysis, the best value to use as the read depth when predicting the MC3 recovery rate of PCAWG variants would be the MC3 read depth at the same site and sample as the PCAWG variant. However, it was not practical to obtain MC3 read depths at sites without MC3 variants, so instead we simulated an MC3 read depth for each PCAWG variant by randomly sampling from the read depths of observed MC3 variants from the same sample as the PCAWG sample. We then matched these simulated read depths for each variant to the closest read depths reported in Fan et al.

For the binned VAFs and read depths for each PCAWG variant obtained as above, we pulled the corresponding sensitivities of MuSE from the Fan et al. paper and simulated MC3 variants with probability equal to these sensitivities.

Integrating clonality. Both consortia considered clonality in their comprehensive characterization of the somatic mutations. Locations of these files are provided in the data availability section. Here, we provide a brief summary of the strategies used to compile these resources. First, the PanCancer Atlas working-groups used MC3 mutations to predict subclonal structures using ABSOLUTE ${ }^{38}$. This tool uses copy number, recurrent karyotype, and mutation data to calculate copy number purity and cluster identification. Furthermore, the PanCancer Atlas working group only made the distinction of clonal and subclonal mutations and did not attempt to further assign sub-clonal mutations to other likely heterogeneous clusters.

PCAWG, on the other hand, used a consensus calling approach incorporating 11 different clustering tools. Here, we evaluated cluster-ID which represents those mutations that are clonal $(\mathrm{ID}=1)$, with other clusters representing mutations that are subclonal (ID $=2$ through 4 ). For this analysis, we restricted our data to SNVs to be consistent with calls made by the PanCancer Atlas calls of MC3 mutations.

Fraction of private variation explained. In Supplementary Fig. 7 we provide a breakdown of different sources of variant described in our analysis using publicly available data. For MC3 all private variants were classified as into 3 variant types (Indel, MissensePlus, and Other). Specifically, indels are comprised of: "Frame_Shift_Del", "In_Frame_Ins", "Frame_Shift_Ins", and "In_Frame_Del". MissensePlus variants are comprised of: "Missense_Mutation", "Nonsense_Mutation", "Nonstop_Mutation", "Splice_Site". And Other variants are comprised of: "RNA", “3'UTR", “5’UTR”, "5'Flank", “Silent”, "3'Flank", "Intron”, "Translation_Start_Site”.

On the other hand, PCAWG variants were also categorized into Indels, MissensePlus, and Other. Specifically, indels are comprised of: "Frame_Shift_Del", "Frame_Shift_Ins", "De_novo_Start_InFrame", "Start_Codon_Ins", "Stop_Codon_Ins", "In_Frame_Del", "In_Frame_Ins", "Stop_Codon_Del", and "Start_Codon_Del". MissensePlus variants are comprised of: "Missense_Mutation", "Nonsense_Mutation", "Nonstop_Mutation", "Splice_Site". And other variants are comprised of: “5'UTR”, "RNA”, “5'Flank”, "Silent”, “3’UTR”, "Intron”, “IGR”,

"lincRNA", "De_novo_Start_OutOfFrame", and "Start_Codon_SNP".

In addition to the three variant type categories, six additional sources of variation were added to private variants: Subclonal, VAF5, VAF10, MMcomplement, THCA KICH or PRAD, and GCcontents. As mentioned, subclonal variants are tagged if labeled as identified by the TCGA or ICGC 
consortia. VAF5 tags all variants with less that $5 \%$ VAF. VAF10 tags all variants with VAF greater than or equal to $5 \%$ and less that $10 \%$. MMcompelement tags all variants not detected by MuSE or MuTect. And finally, GCcontent was calculated as the fraction of $\mathrm{G}$ or $\mathrm{C}$ nucleotides in a $100 \mathrm{bp}$ window surround a variant. This was calculated using a CADD plug-in to VEP. The GCcontent flag was assigned to a variant if $\mathrm{GC}$ fraction was less and 0.3 or greater than 0.7 .

MAFit: online comparison and visualization tool. MAFit (maf Interaction Tool) is a shinyapp ${ }^{39}$ tool to visualize and extract mutations from the intersection of PCAWG and MC3 call sets. It is an interactive and graphical web-based interface built using R Shiny. The interface consists of three panels: a main scatter plot display, a side box of control widgets, and a mutation table in the bottom pane. Any alteration of a control widget will update the scatter plot and the mutation table, enabling very rapid browsing. There is also a button to download the current data set displayed in the scatter plot.

The main panel displays a scatter plot with marginal histograms of mutations grouped by sample. The axes are percentage of matched mutations versus matched plus call-set-unique mutations. Mouse-hovering on a data point initiates a pop-up window showing specific information for this sample, such as TCGA barcode, number of matched mutations, and numbers of mutations unique to each call-set.

The side panel contains two sets of control widgets which can be used to select data based on different criteria. The top control set consists of two sliders to set the VAF cut-offs for each call-set. Both ends of the slider can be adjusted so that users can obtain a desired interval of the VAF. The bottom control set consists of checkboxes of both variant-level and sample-level MC3 filters. If only variant-level filters are checked, all PCAWG-only mutations will be retained; if at least one samplelevel filter is checked, mutations from samples that do not have any checked filters flagged (variant-level or sample-level) will be filtered out. Both variant-level and sample-level filters result in the union of mutations with any checked filter will be shown.

The bottom panel displays a table of mutations based on the selection criteria from the side panel. Columns include information on each mutation, such as TCGA barcode, gene name, VAF, variant class, Human Genome Variant Society (HGVS) nomenclature, etc. Users can sort the table by each column. There are two drop-down selection boxes where users can view mutations from a specific TCGA barcode or Hugo symbol. There is also a search bar, which results in mutations that contain the input in any columns.

Controlled access files. Having worked with both the TCGA and ICGC consortia, we were privy to the controlled access data (all MC3 somatic variant calls and PCAWG germline calls). These data sets allowed for the further interrogation of unique variants called by both groups.

We performed a mutation variant intersection of MC3 controlled access mutations with unique PCAWG variants in the captured exonic regions. These data can be downloaded using necessary credentials from https://gdc.cancer.gov/ about-data/publications/mc3-2017.

We intersected the MC3 public MAF with the PCAWG germline call-set, donor-by-donor. Six MC3 somatic variants were identified as germline variants in PCAWG for the same donor. Of these, five were flagged in MC3 as OxoG or nonpreferred-pair artifacts, and only one was marked PASS in MC3. This PASS variant had a depth in the matched MC3 normal of well over 100 with no alternate reads. The minimal intersection between the MC3 somatic call-set and the donormatched PCAWG germline call-set is evidence that germline contamination in MC3 calls is low.

\section{Assessment of impact on mutation signature analysis. We ran Signature-} Analyzer ${ }^{40}$ on the corpus of WES and WGS samples from our TCGA cases. This tool reports a vector of $J=7$ normalized weights corresponding to mutational signatures. Once weights were computed, we used COSMIC signatures as a reference in order to synchronize labels of the fractional weights between WES and WGS data for each case to enable proper comparison. We excluded 5 cases in which signatures were not computed for WGS data. Using the synchronized results, we then assessed both the number of cases for which the tool identified the same dominant signature between WES and WGS data and also evaluated the correlation between WES and WGS vectors for each case using least-squares regression. Statistical significance of each correlation was calculated using a 2 -tailed $t$-test. Statistical power of each correlation was limited by the paucity of signature weights because the underlying t-statistic is proportional to the square root of $J-2$. However, because these cases, and therefore their hypothesis tests, are independent, the cohort constitutes multiple tests of the same hypothesis regarding signatures derived from WES and WGS data. Therefore, we combined individual P-values into an overall cohort $P$-value using Fisher's log-transform. Namely, the transform of negative 2 times the natural $\log$ of the product of the $K=739$ individual $P$-values is, itself, chi-square distributed with $2 K$ degrees of freedom. Using this transform, we found an overall cohort $P$-value of $<2 \times 10^{-6}$.
Reporting summary. Further information on research design is available in the Nature Research Reporting Summary linked to this article.

\section{Data availability}

Somatic and germline variant calls, mutational signatures, subclonal reconstructions, transcript abundance, splice calls and other core data generated by the ICGC/TCGA Pancancer Analysis of Whole Genomes Consortium are described in another publication ${ }^{10}$ and available for download at https://dcc.icgc.org/releases/PCAWG. Additional information on accessing the data, including raw read files, can be found at https://docs. icgc.org/pcawg/data/. In accordance with the data access policies of the ICGC and TCGA projects, most molecular, clinical and specimen data are in an open tier which does not require access approval. To access potentially identification information, such as germline alleles and underlying sequencing data, researchers will need to apply to the TCGA Data Access Committee (DAC) via dbGaP (https://dbgap.ncbi.nlm.nih.gov/aa/ wga.cgi?page=login) for access to the TCGA portion of the dataset, and to the ICGC Data Access Compliance Office (DACO; http://icgc.org/daco) for the ICGC portion. In addition, to access somatic single nucleotide variants derived from TCGA donors, researchers will also need to obtain $\mathrm{dbGaP}$ authorization. Additional links to data resources used for this project can be found using the following urls: MC3 public MAF file (https://api.gdc.cancer.gov/data/1c8cfe5f-e52d-41ba-94da-f15ea1337efc), PCAWG Public MAF file (https://www.synapse.org/\#!Synapse:syn7364923), bed files used for exome restrictions (MC3) (https://api.gdc.cancer.gov/data/7f0d3ab9-8bef-4e3b-928a6090caae885b), bed files used for exome restrictions (THE BROAD) (https://api.gdc. cancer.gov/data/ble303a5-a542-4389-8ddb-1d151218be75), wiggle files MC3 (https:// www.synapse.org/\#!Synapse:syn21785741), wiggle files PCAWG (https://www.synapse. org/\#!Synapse:syn8492850), clonality files MC3 (https://www.synapse.org/\#!'Synapse: syn7870168), clonality files PCAWG (https://www.synapse.org/\#!Synapse:syn8532460), cancer subtypes and histological data (https://www.synapse.org/\#!Synapse:syn4983466), MAFit online comparison tool (https://mbailey.shinyapps.io/MAFit/), GitHub Repo (https://github.com/ding-lab/mc3_icgc_variant_pipeline), side-by-side MC3-PCAWG comparison (MAF-like) (https://www.synapse.org/\#!Synapse:syn21041380). The remaining data is available within the Article, Supplementary Information or available from the authors upon reasonable request.

\section{Code availability}

A public GitHub repository (under an MIT opensource license) at https://github.com/ ding-lab/mc3_icgc_variant_pipeline furnishes work-flows, scripts, figures, and computational tools used to assess mutation concordance between maf files. For this project we refrained from accessing alignment files, i.e., BAM files or fastq files. Furthermore, due to the memory footprint of mutation and coverage files we have not included them in the repository. Thus, by removing core data files from the repository the software provided supplies the user with processes and decisions, not a fully automated tool for deployment on additional datasets. In addition to our analysis, the core computational pipelines used by the PCAWG Consortium for alignment, quality control and variant calling are available to the public at https://dockstore.org/search? search=pcawg under the GNU General Public License v3.0, which allows for reuse and distribution.

Received: 22 March 2019; Accepted: 28 July 2020; Published online: 21 September 2020

\section{References}

1. Radenbaugh, A. J. et al. RADIA: RNA and DNA integrated analysis for somatic mutation detection. PLoS ONE 9, el11516 (2014).

2. Koboldt, D. C. et al. VarScan 2: Somatic mutation and copy number alteration discovery in cancer by exome sequencing. Genome Res. 22, 568-576 (2012).

3. Fan, Y. et al. MuSE: accounting for tumor heterogeneity using a samplespecific error model improves sensitivity and specificity in mutation calling from sequencing data. Genome Biol. 17, 178 (2016).

4. Cibulskis, K. et al. Sensitive detection of somatic point mutations in impure and heterogeneous cancer samples. Nat. Biotechnol. 31, 213-219 (2013).

5. Ye, K., Schulz, M. H., Long, Q., Apweiler, R. \& Ning, Z. Pindel: a pattern growth approach to detect break points of large deletions and medium sized insertions from paired-end short reads. Bioinformatics 25, 2865-2871 (2009).

6. Ye, K. et al. Systematic discovery of complex insertions and deletions in human cancers. Nat. Med. 22, 97-104 (2016).

7. Chapman, M. A. et al. Initial genome sequencing and analysis of multiple myeloma. Nature 471, 467-472 (2011).

8. Larson, D. E. et al. Somaticsniper: Identification of somatic point mutations in whole genome sequencing data. Bioinformatics 28, 311-317 (2012).

9. Moncunill, V. et al. Comprehensive characterization of complex structural variations in cancer by directly comparing genome sequence reads. Nat. Biotechnol. 32, 1106-1112 (2014). 
10. The ICGC/TCGA Pan-Cancer Analysis of Whole Genomes Network. Pancancer analysis of whole genomes. Nature 578, 82-93 (2019).

11. Sims, D., Sudbery, I., Ilott, N. E., Heger, A. \& Ponting, C. P. Sequencing depth and coverage: key considerations in genomic analyses. Nat. Rev. Genet. 15, 121-132 (2014).

12. Chilamakuri, C. S. R. et al. Performance comparison of four exome capture systems for deep sequencing. BMC Genomics 15, 449 (2014)

13. Belkadi, A. et al. Whole-genome sequencing is more powerful than wholeexome sequencing for detecting exome variants. Proc. Natl Acad. Sci. USA 112, 5473-5478 (2015).

14. Spencer, D. H. et al. Performance of common analysis methods for detecting low-frequency single nucleotide variants in targeted next-generation sequence data. J. Mol. Diagnostics 16, 75-88 (2014).

15. Alfares, A. et al. Whole-genome sequencing offers additional but limited clinical utility compared with reanalysis of whole-exome sequencing. Genet. Med. 20, 1328-1333 (2018).

16. Phallen, J. et al. Early noninvasive detection of response to targeted therapy in non-small cell lung cancer. Cancer Res. 79, 1204-1213 (2019).

17. Głodzik, D. et al. Mutational mechanisms of amplifications revealed by analysis of clustered rearrangements in breast cancers. Ann. Oncol. 29, 2223-2231 (2018).

18. Ren, S. et al. Whole-genome and transcriptome sequencing of prostate cancer identify new genetic alterations driving disease progression [Figure presented]. Eur. Urol. 73, 322-339 (2018).

19. Zhang, S. et al. Genome evolution analysis of recurrent testicular malignant mesothelioma by whole-genome sequencing. Cell. Physiol. Biochem. 45, 163-174 (2018).

20. Nik-Zainal, S. et al. Landscape of somatic mutations in 560 breast cancer whole-genome sequences. Nature 534, 47-54 (2016).

21. Mendoza-Alvarez, A. et al. Whole-exome sequencing identifies somatic mutations associated with mortality in metastatic clear cell kidney carcinoma. Front. Genet. 10, 1-10 (2019).

22. Yan, P. et al. Whole exome sequencing of ulcerative colitis-associated colorectal cancer based on novel somatic mutations identified in Chinese patients. Inflamm. Bowel Dis. 25, 1293-1301 (2019).

23. Mogensen, M. B. et al. Genomic alterations accompanying tumour evolution in colorectal cancer: tracking the differences between primary tumours and synchronous liver metastases by whole-exome sequencing. BMC Cancer 18, 752 (2018)

24. Sponziello, M. et al. Whole exome sequencing identifies a germline MET mutation in two siblings with hereditary wild-type RET medullary thyroid cancer. Hum. Mutat. 39, 371-377 (2018)

25. Schwarze, K., Buchanan, J., Taylor, J. C. \& Wordsworth, S. Are whole-exome and whole-genome sequencing approaches cost-effective? A systematic review of the literature. Genet. Med. 20, 1122-1130 (2018).

26. Choi, M. et al. Genetic diagnosis by whole exome capture and massively parallel DNA sequencing. Proc. Natl Acad. Sci. USA 106, 19096-19101 (2009).

27. Ellrott, K. et al. Scalable open science approach for mutation calling of tumor exomes using multiple genomic pipelines. Cell Syst. 6, 271-281.e7 (2018).

28. Bailey, M. H. et al. Comprehensive characterization of cancer driver genes and mutations. Cell 173, 371-385.e18 (2018).

29. Meienberg, J. et al. New insights into the performance of human whole-exome capture platforms. Nucleic Acids Res. 43, e76 (2015).

30. Clark, M. J. et al. Performance comparison of exome DNA sequencing technologies. Nat. Biotechnol. 29, 908-916 (2011).

31. McLaren, W. et al. Deriving the consequences of genomic variants with the Ensembl API and SNP effect predictor. Bioinformatics 26, 2069-2070 (2010).

32. Kircher, M. et al. A general framework for estimating the relative pathogenicity of human genetic variants. Nat. Genet. 46, 310-315 (2014).

33. Rheinbay, E. et al. Analyses of non-coding somatic drivers in 2,658 cancer whole genomes. Nature 578, 102-111 (2019).

34. Imielinski, M., Guo, G. \& Meyerson, M. Insertions and deletions target lineage-defining genes in human cancers. Cell 168, 460-472.e14 (2017).

35. Dees, N. D. et al. MuSiC: Identifying mutational significance in cancer genomes. Genome Res. 22, 1589-1598 (2012).

36. Auslander, N. et al. An integrated computational and experimental study uncovers FUT 9 as a metabolic driver of colorectal cancer. Mol. Syst. Biol. 13, 956 (2017)

37. Neph, S. et al. BEDOPS: High-performance genomic feature operations. Bioinformatics 28, 1919-1920 (2012).
38. Carter, S. L. et al. Absolute quantification of somatic DNA alterations in human cancer. Nat. Biotechnol. 30, 413-421 (2012).

39. Chang, W., Cheng, J., Allaire, J., Xie, Y. \& McPherson, J. shiny: Web Application Framework for R. R Packag (2016)

40. Kasar, $\mathrm{S}$. et al. Whole-genome sequencing reveals activation-induced cytidine deaminase signatures during indolent chronic lymphocytic leukaemia evolution. Nat. Commun. 6, 1-12 (2015).

41. Conway, J. R., Lex, A. \& Gehlenborg, N. UpSetR: An R package for the visualization of intersecting sets and their properties. Bioinformatics 33, 2938-2940 (2017)

42. Gu, Z., Eils, R. \& Schlesner, M. Complex heatmaps reveal patterns and correlations in multidimensional genomic data. Bioinformatics. https://doi. org/10.1093/bioinformatics/btw313 (2016).

\section{Acknowledgements}

We acknowledge the contributions of the many clinical networks across ICGC and TCGA who provided samples and data to the PCAWG Consortium and the contributions of the Technical Working Group and the Germline Working Group of the PCAWG Consortium for collation, realignment, and harmonization of the variant calls of the cancer genomes used by this study. We thank the patients and their families for their participation in the individual ICGC and TCGA projects.

\section{Author contributions}

L.D. and M.H.B. conceived the project. L.D. and M.B.G. supervised the project. M.H.B., W.M., and G.D. drafted the manuscript. M.H.B., L.-B.W., W.M., S.L., G.S., M.C.W., G.D., M.B.G., L.D., D.A.W., J.T.S., G.G., and L.J.D. provided scientific input. G.S. and K.E provided sample mapping and quality control files. T.C.G.A.R.N. and P.C organized, generated, and distributed raw data. P.C. and N.S.M.C.M. produced original wholegenome variant calls. M.H.B., W.M., G.D., and L.-B.W. produced figures. Analysis was performed by M.H.B., W.M., L.-B.W., G.D., Y.L., W.-W.L., M.C.W., and A.W. All authors approve the submission of this manuscript. We thank S.K. for providing text edits.

\section{Competing interests}

The authors declare the following competing interests: G.G. receives research funds from IBM and Pharmacyclics. G.G. is an inventor on patent applications related to MuTect, ABSOLUTE, and other tools. The remaining authors declare no competing interests.

\section{Additional information}

Supplementary information is available for this paper at https://doi.org/10.1038/s41467020-18151-y.

Correspondence and requests for materials should be addressed to M.B.G. or L.D.

Peer review information Nature Communications thanks the anonymous reviewer(s) for their contribution to the peer review of this work.

Reprints and permission information is available at http://www.nature.com/reprints

Publisher's note Springer Nature remains neutral with regard to jurisdictional claims in published maps and institutional affiliations.

Open Access This article is licensed under a Creative Commons Attribution 4.0 International License, which permits use, sharing, adaptation, distribution and reproduction in any medium or format, as long as you give appropriate credit to the original author(s) and the source, provide a link to the Creative Commons license, and indicate if changes were made. The images or other third party material in this article are included in the article's Creative Commons license, unless indicated otherwise in a credit line to the material. If material is not included in the article's Creative Commons license and your intended use is not permitted by statutory regulation or exceeds the permitted use, you will need to obtain permission directly from the copyright holder. To view a copy of this license, visit http://creativecommons.org/ licenses/by/4.0/.

(C) The Author(s) 2020, corrected publication 2020 


\section{MC3 Working Group}

Rehan Akbani ${ }^{21}$, Pavana Anur ${ }^{22}$, Matthew H. Bailey ${ }^{1,2,3}$, Alex Buchanan ${ }^{9}$, Kami Chiotti ${ }^{9}$, Kyle Covington ${ }^{12,23}$,

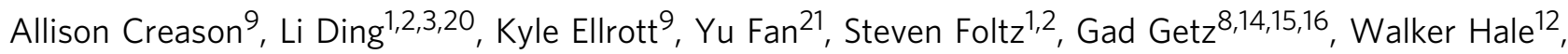
David Haussler 24,25, Julian M. Hess ${ }^{8,26}$, Carolyn M. Hutter ${ }^{27}$, Cyriac Kandoth ${ }^{28}$, Katayoon Kasaian 29,30, Melpomeni Kasapi ${ }^{27}$, Dave Larson', Ignaty Leshchiner ${ }^{8}$, John Letaw ${ }^{31}$, Singer Ma ${ }^{32}$, Michael D. McLellan ${ }^{1,3,20}$, Yifei Men ${ }^{32}$, Gordon B. Mills ${ }^{33,34}$, Beifang Niu ${ }^{35}$, Myron Peto ${ }^{22}$, Amie Radenbaugh ${ }^{24}$, Sheila M. Reynolds ${ }^{36}$, Gordon Saksena ${ }^{8}$, Heidi Sofia ${ }^{27}$, Chip Stewart ${ }^{8}$, Adam J. Struck ${ }^{31}$, Joshua M. Stuart ${ }^{24,37}$, Wenyi Wang ${ }^{21}$, John N. Weinstein ${ }^{38}$, David A. Wheeler ${ }^{12,13}$, Christopher K. Wong ${ }^{24,39}$, Liu Xi' ${ }^{12} \&$ Kai Ye 40,41

\footnotetext{
${ }^{21}$ Department of Bioinformatics and Computational Biology, The University of Texas MD Anderson Cancer Center, Houston, TX 77030, USA. ${ }^{22}$ Molecular and Medical Genetics, OHSU Knight Cancer Institute, Oregon Health and Science University, Portland, OR 97239, USA. ${ }^{23}$ Castle Biosciences Inc, Friendswood, TX 77546, USA. ${ }^{24}$ UC Santa Cruz Genomics Institute, University of California Santa Cruz, Santa Cruz, CA 95064, USA. ${ }^{25}$ Howard Hughes Medical Institute, University of California Santa Cruz, Santa Cruz, CA 95064, USA. ${ }^{26}$ Massachusetts General Hospital Center for Cancer Research, Charlestown, MA 02114, USA. ${ }^{27}$ National Human Genome Research Institute, National Institutes of Health, Bethesda, MD 20894, USA. ${ }^{28}$ Marie-Josée and Henry R. Kravis Center for Molecular Oncology, Memorial Sloan Kettering Cancer Center, New York, NY 10065, USA. ${ }^{29}$ Ontario Institute for Cancer Research, Toronto, ON M5G OA3, Canada. ${ }^{30}$ Canada's Michael Smith Genome Sciences Centre, BC Cancer, Vancouver, BC V5Z 4S6, Canada. ${ }^{31}$ Computational Biology Program, School of Medicine, Oregon Health and Science University, Portland, OR 97239, USA. ${ }^{32}$ DNAnexus Inc, Mountain View, CA 94040, USA. ${ }^{33}$ Department of Systems Biology, UT MD Anderson Cancer Center, Houston, TX 77030, USA. ${ }^{34}$ Precision Oncology, OHSU Knight Cancer Institute, Oregon Health and Science University, Portland, OR 97239, USA. ${ }^{35}$ Computer Network Information Center, Chinese Academy of Sciences, Beijing, China. ${ }^{36}$ Institute for Systems Biology, Seattle, WA 98109, USA. ${ }^{37}$ Department of Biomolecular Engineering, University of California Santa Cruz, Santa Cruz, CA 95064, USA. ${ }^{38}$ Department of Bioinformatics and Computational Biology and Department of Systems Biology, The University of Texas MD Anderson Cancer Center, Houston, TX 77030, USA.

${ }^{39}$ Biomolecular Engineering Department, University of California Santa Cruz, Santa Cruz, CA 95064, USA. ${ }^{40}$ School of Electronic and Information Engineering, Xi'an Jiaotong University, Xi'an, China. ${ }^{41}$ The First Affiliated Hospital, Xi'an Jiaotong University, Xi'an, China.
}

\section{PCAWG novel somatic mutation calling methods working group}

Matthew H. Bailey 1,2,3, Beifang Niu ${ }^{35}$, Matthias Bieg42,43, Paul C. Boutros $6,44,45,46$, Ivo Buchhalter 43,47,48, Adam P. Butler ${ }^{49}$, Ken Chen50, Zechen Chong51, Li Ding1,2,3,20, Oliver Drechsel ${ }^{52,53}$, Lewis Jonathan Dursi ${ }^{6,7}$, Roland Eils $47,48,54,55$, Kyle Ellrott ${ }^{9}$, Shadrielle M. G. Espiritu ${ }^{6}$, Yu Fan ${ }^{21}$, Robert S. Fulton 1,3,20, Shengjie Gao ${ }^{56}$, Josep L. I. Gelpi ${ }^{57,58}$, Mark B. Gerstein 5,18,19, Gad Getz $8,14,15,16$, Santiago Gonzalez ${ }^{59,60}$, Ivo G. Gut ${ }^{52,61}$, Faraz Hach ${ }^{62,63}$, Michael C. Heinold ${ }^{47,48}$, Julian M. Hess ${ }^{8,26}$, Jonathan Hinton ${ }^{49}$, Taobo Hu ${ }^{64}$, Vincent Huang6, Yi Huang 65,66, Barbara Hutter ${ }^{43,67,68}$, David R. Jones ${ }^{49}$, Jongsun Jung ${ }^{69}$, Natalie Jäger ${ }^{47}$, Hyung-Lae Kim ${ }^{70}$, Kortine Kleinheinz ${ }^{47,48}$, Sushant Kumar ${ }^{5,19}$, Yogesh Kumar ${ }^{64}$, Christopher M. Lalansingh ${ }^{6}$, Ignaty Leshchiner ${ }^{8}$, Ivica Letunic ${ }^{71}$, Dimitri Livitz ${ }^{8}$, Eric Z. Ma64, Yosef E. Maruvka $8,26,72$, R. Jay Mash1 ${ }^{1,2}$, Michael D. McLellan ${ }^{1,3,20}$, Andrew Menzies ${ }^{49}$, Ana Milovanovic ${ }^{57}$, Morten Muhlig Nielsen ${ }^{73}$, Stephan Ossowski ${ }^{52,53,74,}$ Nagarajan Paramasivam 43,47, Jakob Skou Pedersen ${ }^{73,75}$, Marc D. Perry ${ }^{76,77}$, Montserrat Puiggròs ${ }^{57}$, Keiran M. Raine ${ }^{49}$, Esther Rheinbay ${ }^{8,14,72}$, Romina Royo 57 , S. Cenk Sahinalp ${ }^{62,78,79}$, Gordon Saksena ${ }^{8}$, Iman Sarrafi62,78, Matthias Schlesner ${ }^{47,80}$, Jared T. Simpson 6,17, Lucy Stebbings ${ }^{49}$, Chip Stewart ${ }^{8}$, Miranda D. Stobbe ${ }^{52,61}$, Jon W. Teague ${ }^{49}$, Grace Tiao ${ }^{8}$, David Torrents ${ }^{57,81}$, Jeremiah A. Wala $8,14,82$, Jiayin Wang 1,40,66, Wenyi Wang ${ }^{21}$, Sebastian M. Waszak ${ }^{60}$, Joachim Weischenfeldt $60,83,84$, Michael C. Wendl 1,10,11, Johannes Werner ${ }^{47,85}$, Zhenggang Wu ${ }^{64}$, Hong Xue ${ }^{64}$, Sergei Yakneen ${ }^{60}$, Takafumi N. Yamaguchi6, Kai Ye ${ }^{40,41}$, Venkata D. Yellapantula 20,86 , Christina K. Yung ${ }^{76}$ \& Junjun Zhang ${ }^{76}$

\footnotetext{
${ }^{42}$ Center for Digital Health, Berlin Institute of Health and Charitè-Universitätsmedizin Berlin, 10178 Berlin, Germany. ${ }^{43}$ Heidelberg Center for Personalized Oncology (DKFZ-HIPO), German Cancer Research Center (DKFZ), 69120 Heidelberg, Germany. ${ }^{44}$ Department of Medical Biophysics, University of Toronto, Toronto, ON M5S, Canada. ${ }^{45}$ Department of Human Genetics, University of California Los Angeles, Los Angeles, CA 90095, USA. ${ }^{46}$ Department of Pharmacology, University of Toronto, Toronto, ON M5S, Canada. ${ }^{47}$ Division of Theoretical Bioinformatics, German Cancer Research Center (DKFZ), 69120 Heidelberg, Germany. ${ }^{48}$ Institute of Pharmacy and Molecular Biotechnology and BioQuant, Heidelberg University, 69117 Heidelberg, Germany. ${ }^{49}$ Wellcome Sanger Institute, Wellcome Genome Campus, Hinxton CB10 1SA, UK. ${ }^{50}$ University of Texas MD Anderson Cancer Center, Houston, TX 77030, USA. ${ }^{51}$ Department of Genetics, Informatics Institute, University of Alabama at Birmingham, Birmingham, AL 77030, USA. ${ }^{52}$ Universitat Pompeu Fabra (UPF), 08002 Barcelona, Spain. ${ }^{53}$ Centre for Genomic Regulation (CRG), The Barcelona Institute of Science and Technology, Barcelona, Spain. ${ }^{54}$ Heidelberg University, 69117 Heidelberg, Germany. ${ }^{55}$ New BIH Digital Health Center, Berlin Institute of Health (BIH) and Charité-Universitätsmedizin Berlin, 08036 Berlin, Germany. ${ }^{56} \mathrm{BGI}-$ Shenzhen, Shenzhen, China.
} 
${ }^{57}$ Barcelona Supercomputing Center (BSC), 08034 Barcelona, Spain. ${ }^{58}$ Department Biochemistry and Molecular Biomedicine, University of Barcelona, 08007 Barcelona, Spain. ${ }^{59}$ European Molecular Biology Laboratory, European Bioinformatics Institute (EMBL-EBI), Cambridge CB10 1SD, UK. ${ }^{60}$ Genome Biology Unit, European Molecular Biology Laboratory (EMBL), 22607 Heidelberg, Germany. ${ }^{61}$ CNAG-CRG, Centre for Genomic Regulation (CRG), Barcelona Institute of Science and Technology (BIST), 08036 Barcelona, Spain. ${ }^{62}$ Vancouver Prostate Centre, Vancouver, BC V6H 3Z6, Canada. ${ }^{63}$ Department of Urologic Sciences, University of British Columbia, Vancouver, BC V6T 1Z4, Canada. ${ }^{64}$ Division of Life Science and Applied Genomics Center, Hong Kong University of Science and Technology, Clear Water Bay, Hong Kong, China. ${ }^{65}$ GeneplusShenzhen, Shenzhen, China. ${ }^{66}$ School of Computer Science and Technology, Xi'an Jiaotong University, Xi'an, China. ${ }^{67}$ National Center for Tumor Diseases (NCT) Heidelberg, 69120 Heidelberg, Germany. ${ }^{68}$ German Cancer Consortium (DKTK), 69120 Heidelberg, Germany. ${ }^{69}$ Genome Integration Data Center, Syntekabio, Inc, Daejeon, South Korea. ${ }^{70}$ Department of Biochemistry, College of Medicine, Ewha Womans University, Seoul, South Korea. ${ }^{71}$ Biobyte solutions GmbH, 69126 Heidelberg, Germany. ${ }^{72}$ Massachusetts General Hospital, Boston, MA 02114, USA.

${ }^{73}$ Department of Molecular Medicine (MOMA), Aarhus University Hospital, 8200 Aarhus N, Denmark. ${ }^{74}$ Institute of Medical Genetics and Applied Genomics, University of Tübingen, 72074 Tübingen, Germany. ${ }^{75}$ Bioinformatics Research Centre (BiRC), Aarhus University, 8000 Aarhus, Denmark. ${ }^{76}$ Genome Informatics Program, Ontario Institute for Cancer Research, Toronto, ON M5G 0A3, Canada. ${ }^{77}$ Department of Radiation Oncology, University of California San Francisco, San Francisco, CA 94110, USA. ${ }^{78}$ Simon Fraser University, Burnaby, BC V5A 1S6, Canada. ${ }^{79}$ Indiana University, Bloomington, IN 47405, USA. ${ }^{80}$ Bioinformatics and Omics Data Analytics, German Cancer Research Center (DKFZ), 69120 Heidelberg, Germany. ${ }^{81}$ Institució Catalana de Recerca i Estudis Avançats (ICREA), Barcelona, Spain. ${ }^{82}$ Department of Medical Oncology, Dana-Farber Cancer Institute, Boston, MA 02215, USA. ${ }^{83}$ Finsen Laboratory and Biotech Research and Innovation Centre (BRIC), University of Copenhagen, 1165 Copenhagen, Denmark. ${ }^{84}$ Department of Urology, Charité Universitätsmedizin Berlin, 10117 Berlin, Germany. ${ }^{85}$ Department of Biological Oceanography, Leibniz Institute of Baltic Sea Research, 18119 Rostock, Germany. ${ }^{86}$ Department of Epidemiology and Biostatistics, Memorial Sloan Kettering Cancer Center, New York, NY 10065, USA.

\section{PCAWG Consortium}

Lauri A. Aaltonen 87 , Federico Abascal ${ }^{49}$, Adam Abeshouse 88 , Hiroyuki Aburatani89, David J. Adams ${ }^{49}$, Nishant Agrawal90, Keun Soo Ahn91, Sung-Min Ahn92, Hiroshi Aikata93, Rehan Akbani ${ }^{21}$, Kadir C. Akdemir ${ }^{50}$, Hikmat Al-Ahmadie ${ }^{88}$, Sultan T. Al-Sedairy 94 , Fatima Al-Shahrour 95 , Malik Alawi 96,97 , Monique Albert ${ }^{98,}$ Kenneth Aldape 99,100, Ludmil B. Alexandrov49,101,102, Adrian Ally30, Kathryn Alsop ${ }^{103}$, Eva G. Alvarez 104,105,106, Fernanda Amary ${ }^{107}$, Samirkumar B. Amin 108,109,110, Brice Aminou ${ }^{76}$, Ole Ammerpohl111,112,

Matthew J. Anderson ${ }^{113}$, Yeng Ang ${ }^{114}$, Davide Antonello ${ }^{115}$, Pavana Anur 22 , Samuel Aparicio ${ }^{116}$, Elizabeth L. Appelbaum ${ }^{1117}$, Yasuhito Arai ${ }^{118}$, Axel Aretz ${ }^{119}$, Koji Arihiro93, Shun-ichi Ariizumi ${ }^{120}$, Joshua Armenia ${ }^{121}$, Laurent Arnould ${ }^{122}$, Sylvia Asa123,124, Yassen Assenov ${ }^{125}$, Gurnit Atwal6,126,127, Sietse Aukema ${ }^{112,128}$, J. Todd Auman ${ }^{129}$, Miriam R. Aure ${ }^{130}$, Philip Awadalla6,126, Marta Aymerich ${ }^{131}$, Gary D. Bader ${ }^{126}$, Adrian Baez-Ortega ${ }^{132}$, Matthew H. Bailey 1,2,3, Peter J. Bailey ${ }^{133}$, Miruna Balasundaram ${ }^{30}$, Saianand Balu ${ }^{134}$, Pratiti Bandopadhayay $8,135,136$, Rosamonde E. Banks ${ }^{137}$, Stefano Barbi ${ }^{138}$, Andrew P. Barbour 139,140, Jonathan Barenboim6 ${ }^{6}$, Jill Barnholtz-Sloan ${ }^{141,142}$, Hugh Barr ${ }^{143}$, Elisabet Barrera ${ }^{59}$, John Bartlett 98,144 , Javier Bartolome ${ }^{57}$, Claudio Bassi ${ }^{115}$, Oliver F. Bathe ${ }^{145,146}$, Daniel Baumhoer ${ }^{147}$, Prashant Bavi ${ }^{148}$, Stephen B. Baylin ${ }^{149,150}$, Wojciech Bazant ${ }^{59}$, Duncan Beardsmore ${ }^{151}$, Timothy A. Beck ${ }^{152,153}$, Sam Behjati ${ }^{49}$, Andreas Behren ${ }^{154}$, Beifang Niu ${ }^{35}$, Cindy Bell ${ }^{155}$, Sergi Beltran ${ }^{52,61}$, Christopher Benz ${ }^{156}$, Andrew Berchuck 157, Anke K. Bergmann ${ }^{158}$, Erik N. Bergstrom 101,102, Benjamin P. Berman 159,160,161, Daniel M. Berney ${ }^{162}$, Stephan H. Bernhart ${ }^{163,164,165}$, Rameen Beroukhim ${ }^{8,14,82}$, Mario Berrios ${ }^{166}$, Samantha Bersani ${ }^{167}$, Johanna Bert1 ${ }^{73,168}$, Miguel Betancourt ${ }^{169}$, Vinayak Bhandari 6,44, Shriram G. Bhosle ${ }^{49}$, Andrew V. Biankin 133,170,171,172, Matthias Bieg42,43, Darell Bigner ${ }^{173}$, Hans Binder ${ }^{163,164}$, Ewan Birney ${ }^{59}$, Michael Birrer ${ }^{72}$, Nidhan K. Biswas ${ }^{174}$, Bodil Bjerkehagen ${ }^{147,175}$, Tom Bodenheimer ${ }^{134}$, Lori Boice ${ }^{176}$, Giada Bonizzato ${ }^{177}$, Johann S. De Bono ${ }^{178}$, Arnoud Boot ${ }^{179,180}$, Moiz S. Bootwalla ${ }^{166}$, Ake Borg ${ }^{181}$, Arndt Borkhardt ${ }^{182}$, Keith A. Boroevich ${ }^{183,184}$, Ivan Borozan ${ }^{6}$, Christoph Borst ${ }^{185}$, Marcus Bosenberg ${ }^{186}$, Mattia Bosio 52,53,57, Jacqueline Boultwood ${ }^{187}$, Guillaume Bourque ${ }^{188,189}$, Paul C. Boutros 6,44,45,46, G. Steven Bova190, David T. Bowen 49,191, Reanne Bowlby ${ }^{30}$, David D. L. Bowtell ${ }^{103}$, Sandrine Boyault ${ }^{192}$, Rich Boyce ${ }^{59}$, Jeffrey Boyd ${ }^{193}$, Alvis Brazma59, Paul Brennan ${ }^{194}$, Daniel S. Brewer ${ }^{195,196}$, Arie B. Brinkman ${ }^{197}$, Robert G. Bristow44,198,199,200,201, Russell R. Broaddus99, Jane E. Brock ${ }^{202}$, Malcolm Brock ${ }^{203}$, Annegien Broeks ${ }^{204}$, Angela N. Brooks $8,24,37,82$, Denise Brooks ${ }^{30}$, Benedikt Brors 67,205,206, Søren Brunak ${ }^{207,208}$, Timothy J. C. Bruxner 113,209, Alicia L. Bruzos ${ }^{104,105,106}$, Alex Buchanan99, Ivo Buchhalter 43,47,48, 
Christiane Buchholz 210 , Susan Bullman ${ }^{8,82}$, Hazel Burke 211 , Birgit Burkhardt ${ }^{212}$, Kathleen H. Burns 213,214 , John Busanovich8,215, Carlos D. Bustamante 216,217, Adam P. Butler ${ }^{49}$, Atul J. Butte ${ }^{218}$, Niall J. Byrne ${ }^{76}$, Anne-Lise Børresen-Dale 130,219, Samantha J. Caesar-Johnson 220, Andy Cafferkey ${ }^{59}$, Declan Cahill ${ }^{221}$, Claudia Calabrese ${ }^{59,60}$, Carlos Caldas ${ }^{222,223}$, Fabien Calvo ${ }^{224}$, Niedzica Camacho ${ }^{178}$, Peter J. Campbell ${ }^{49,225}$, Elias Campo 226,227, Cinzia Cantù 177, Shaolong Cao ${ }^{21}$, Thomas E. Carey 228, Joana Carlevaro-Fita229,230,231, Rebecca Carlsen ${ }^{30}$, Ivana Cataldo ${ }^{167,177}$, Mario Cazzola ${ }^{232}$, Jonathan Cebon ${ }^{154}$, Robert Cerfolio ${ }^{233}$,

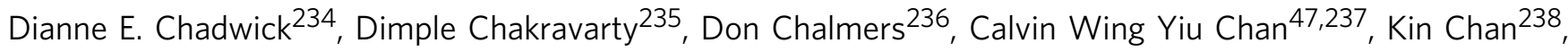

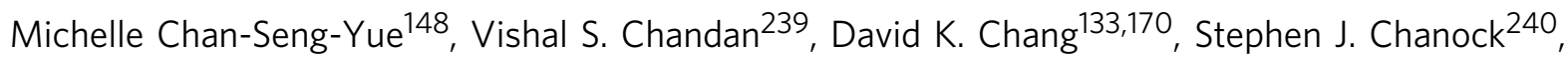
Lorraine A. Chantrill170,241, Aurélien Chateigner ${ }^{76,242}$, Nilanjan Chatterjee ${ }^{149,243}$, Kazuaki Chayama93, Hsiao-Wei Chen ${ }^{114,121}$, Jieming Chen ${ }^{218}$, Ken Chen ${ }^{50}$, Yiwen Chen ${ }^{21}$, Zhaohong Chen ${ }^{244}$, Andrew D. Cherniack ${ }^{8,82}$, Jeremy Chien ${ }^{245}$, Yoke-Eng Chiew ${ }^{246,247}$, Suet-Feung Chin ${ }^{222,223}$, Juok Cho ${ }^{8}$, Sunghoon Cho ${ }^{248}$, Jung Kyoon Choi ${ }^{249}$, Wan Choi ${ }^{250}$, Christine Chomienne ${ }^{251}$, Zechen Chong ${ }^{51}$, Su Pin Choo ${ }^{252}$, Angela Chou ${ }^{170,246}$, Angelika N. Christ ${ }^{113}$, Elizabeth L. Christie ${ }^{103}$, Eric Chuah ${ }^{30}$, Carrie Cibulskis ${ }^{8}$, Kristian Cibulskis $^{8}$, Sara Cingarlini ${ }^{253}$, Peter Clapham ${ }^{49}$, Alexander Claviez ${ }^{254}$, Sean Cleary ${ }^{148,255}$, Nicole Cloonan ${ }^{256}$, Marek Cmero $257,258,259$, Colin C. Collins ${ }^{62}$, Ashton A. Connor ${ }^{255,260,}$ Susanna L. Cooke ${ }^{133}$, Colin S. Cooper ${ }^{178,196,261}$, Leslie Cope ${ }^{149}$, Vincenzo Corbo ${ }^{138,177}$, Matthew G. Cordes ${ }^{1,262}$, Stephen M. Cordner 263 , Isidro Cortés-Ciriano 264,265,266, Kyle Covington ${ }^{12,23}$, Prue A. Cowin ${ }^{267}$, Brian Craft ${ }^{24}$, David Craft 8,268, Chad J. Creighton269, Yupeng Cun ${ }^{270}$, Erin Curley ${ }^{271}$, Ioana Cutcutache ${ }^{179,180,}$ Karolina Czajka ${ }^{272}$, Bogdan Czerniak ${ }^{99,273}$, Rebecca A. Dagg ${ }^{274}$, Ludmila Danilova ${ }^{149}$, Maria Vittoria Davi ${ }^{275}$, Natalie R. Davidson276,277,278,279,280, Helen Davies49,281,282, lan J. Davis ${ }^{283}$, Brandi N. Davis-Dusenbery ${ }^{284}$, Kevin J. Dawson ${ }^{49}$, Francisco M. De La Vega 216,217,285, Ricardo De Paoli-Iseppi ${ }^{211}$, Timothy Defreitas ${ }^{8}$, Angelo P. Dei Tos ${ }^{286}$, Olivier Delaneau 287,288,289, John A. Demchok ${ }^{220}$, Jonas Demeulemeester ${ }^{290,291}$, German M. Demidov52,53,74, Deniz Demircioğlu292,293, Nening M. Dennis ${ }^{221}$, Robert E. Denroche ${ }^{148}$, Stefan C. Dentro 49,290,294, Nikita Desai ${ }^{76}$, Vikram Deshpande ${ }^{72}$, Amit G. Deshwar ${ }^{295}$, Christine Desmedt 296,297, Jordi Deu-Pons 298,299 , Noreen Dhalla ${ }^{30}$, Neesha C. Dhani ${ }^{300,}$ Priyanka Dhingra 301,302, Rajiv Dhir ${ }^{303}$, Anthony DiBiase ${ }^{304}$, Klev Diamanti ${ }^{305}$, Li Ding1,2,3,20, Shuai Ding 306, Huy Q. Dinh ${ }^{159}$, Luc Dirix ${ }^{307}$, HarshaVardhan Doddapaneni ${ }^{12}$, Nilgun Donmez ${ }^{62,78}$, Michelle T. Dow ${ }^{244}$, Ronny Drapkin 308 , Oliver Drechsel ${ }^{52,53}$, Ruben M. Drews ${ }^{223}$, Serge Serge ${ }^{49}$, Tim Dudderidge 150,221, Ana Dueso-Barroso ${ }^{57}$, Andrew J. Dunford ${ }^{8}$, Michael Dunn ${ }^{309}$, Lewis Jonathan Dursi 6,7, Fraser R. Duthie ${ }^{133,310,}$ Ken Dutton-Regester ${ }^{311}$, Jenna Eagles ${ }^{272}$, Douglas F. Easton ${ }^{312,313}$, Stuart Edmonds 314 , Paul A. Edwards 223,315, Sandra E. Edwards ${ }^{178}$, Rosalind A. Eeles ${ }^{178,221}$, Anna Ehinger ${ }^{316}$, Juergen Eils ${ }^{54,55}$, Roland Eils 47,48,54,55,

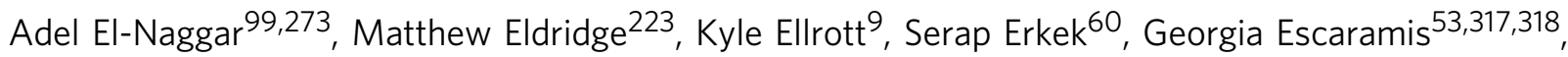
Shadrielle M. G. Espiritu6 ${ }^{6}$ Xavier Estivill ${ }^{53,319}$, Dariush Etemadmoghadam ${ }^{103}$, Jorunn E. Eyfjord ${ }^{320}$, Bishoy M. Faltas ${ }^{280}$, Daiming Fan ${ }^{321}$, Yu Fan 21, William C. Faquin ${ }^{72}$, Claudiu Farcas ${ }^{244}$, Matteo Fassan ${ }^{322,}$ Aquila Fatima ${ }^{323}$, Francesco Favero ${ }^{324}$, Nodirjon Fayzullaev ${ }^{76}$, Ina Felau ${ }^{220}$, Sian Fereday ${ }^{103}$, Martin L. Ferguson 325 , Vincent Ferretti ${ }^{76,326}$, Lars Feuerbach ${ }^{205}$, Matthew A. Field ${ }^{327}$, J. Lynn Fink ${ }^{57,113}$, Gaetano Finocchiaro $^{328}$, Cyril Fisher ${ }^{221}$, Matthew W. Fittall ${ }^{290}$, Anna Fitzgerald ${ }^{329}$, Rebecca C. Fitzgerald ${ }^{282}$, Adrienne M. Flanagan ${ }^{330}$, Neil E. Fleshner ${ }^{331}$, Paul Flicek ${ }^{59}$, John A. Foekens ${ }^{332}$, Kwun M. Fong ${ }^{333}$, Nuno A. Fonseca 59,334 , Christopher S. Foster 335,336 , Natalie S. Fox ${ }^{6}$, Michael Fraser ${ }^{6}$, Scott Frazer ${ }^{8}$, Milana Frenkel-Morgenstern ${ }^{337}$, William Friedman ${ }^{338}$, Joan Frigola ${ }^{298}$, Catrina C. Fronick ${ }^{1,262}$, Akihiro Fujimoto ${ }^{184}$, Masashi Fujita ${ }^{184}$, Masashi Fukayama339, Lucinda A. Fulton', Robert S. Fulton 1,3,20,

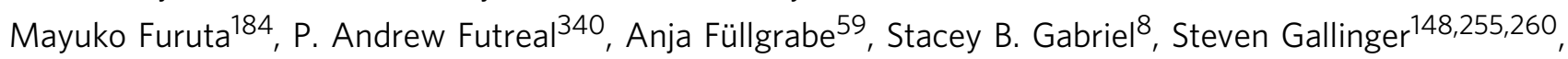
Carlo Gambacorti-Passerini ${ }^{341}$, Jianjiong Gao ${ }^{121}$, Shengjie Gao ${ }^{56}$, Levi Garraway ${ }^{82}$, Øystein Garred ${ }^{342}$, 
Erik Garrison ${ }^{49}$, Dale W. Garsed ${ }^{103}$, Nils Gehlenborg 8,343 , Josep L. I. Gelpi ${ }^{57,58}$, Joshy George ${ }^{110}$, Daniela S. Gerhard ${ }^{344}$, Clarissa Gerhauser ${ }^{345}$, Jeffrey E. Gershenwald 346,347 , Mark B. Gerstein 5,18,19, Moritz Gerstung 59,60, Gad Getz 8 14,15,16, Mohammed Ghori ${ }^{49}$, Ronald Ghossein ${ }^{348}$, Nasra H. Giama ${ }^{349}$, Richard A. Gibbs ${ }^{12}$, Anthony J. Gill170,350, Pelvender Gill ${ }^{351}$, Dilip D. Giri ${ }^{348}$, Dominik Glodzik ${ }^{49}$, Vincent J. Gnanapragasam 352,353, Maria Elisabeth Goebler ${ }^{354}$, Mary J. Goldman 24 , Carmen Gomez ${ }^{355}$, Santiago Gonzalez 59,60, Abel Gonzalez-Perez ${ }^{298,299,356}$, Dmitry A. Gordenin 357, James Gossage ${ }^{358}$, Kunihito Gotoh ${ }^{359}$, Ramaswamy Govindan³, Dorthe Grabau ${ }^{360}$, Janet S. Graham ${ }^{133,361}$, Robert C. Grant ${ }^{148,260}$, Anthony R. Green ${ }^{315}$, Eric Green ${ }^{27}$, Liliana Greger ${ }^{59}$, Nicola Grehan ${ }^{282}$, Sonia Grimaldi ${ }^{177}$, Sean M. Grimmond ${ }^{362}$, Robert L. Grossman ${ }^{363}$, Adam Grundhoff 97,364 , Gunes Gundem 88 , Qianyun Guo ${ }^{75}$, Manaswi Gupta ${ }^{8}$, Shailja Gupta ${ }^{365}$, Ivo G. Gut ${ }^{52,61}$, Marta Gut ${ }^{52,61}$, Jonathan Göke 292,366 , Gavin Ha ${ }^{8}$, Andrea Haake ${ }^{111}$, David Haan ${ }^{37}$, Siegfried Haas ${ }^{185}$, Kerstin Haase ${ }^{290}$, James E. Haber ${ }^{367}$, Nina Habermann ${ }^{60}$, Faraz Hach ${ }^{62,63}$, Syed Haider6, Natsuko Hama ${ }^{118}$, Freddie C. Hamdy ${ }^{351}$, Anne Hamilton ${ }^{267}$, Mark P. Hamilton ${ }^{368}$, Leng Han ${ }^{369}$, George B. Hanna 370 , Martin Hansmann 371 , Nicholas J. Haradhvala8,72, Olivier Harismendy 102,372, Ivon Harliwong ${ }^{113}$, Arif O. Harmanci5,373, Eoghan Harrington ${ }^{374}$, Takanori Hasegawa ${ }^{375}$, David Haussler 24,25, Steve Hawkins ${ }^{223}$, Shinya Hayami ${ }^{376}$, Shuto Hayashi ${ }^{375}$, D. Neil Hayes ${ }^{134,377,378}$, Stephen J. Hayes ${ }^{379,380,}$ Nicholas K. Hayward ${ }^{211,311}$, Steven Hazell ${ }^{221}$, Yao He $\mathrm{H}^{381}$, Allison P. Heath ${ }^{382}$, Simon C. Heath ${ }^{52,61}$, David Hedley ${ }^{300}$, Apurva M. Hegde ${ }^{38}$, David I. Heiman ${ }^{8}$, Michael C. Heinold ${ }^{47,48}$, Zachary Heins ${ }^{88}$, Lawrence E. Heisler ${ }^{152}$, Eva Hellstrom-Lindberg ${ }^{383}$, Mohamed Helmy ${ }^{384}$, Seong Gu Heo ${ }^{385}$, Austin J. Hepperla134, José María Heredia-Genestar ${ }^{386}$, Carl Herrmann47,48,387, Peter Hersey 211 , Julian M. Hess ${ }^{8,26}$, Holmfridur Hilmarsdottir ${ }^{320}$, Jonathan Hinton ${ }^{49}$, Satoshi Hirano ${ }^{388}$, Nobuyoshi Hiraoka ${ }^{389}$, Katherine A. Hoadley ${ }^{134,390}$, Asger Hobolth ${ }^{75,168}$, Ermin Hodzic ${ }^{78}$, Jessica I. Hoell ${ }^{182}$, Steve Hoffmann $163,164,165,391$, Oliver Hofmann ${ }^{392}$, Andrea Holbrook ${ }^{166}$, Aliaksei Z. Holik ${ }^{53}$, Michael A. Hollingsworth ${ }^{393}$, Oliver Holmes 209,311, Robert A. Holt ${ }^{30}$, Chen Hong 205,237, Eun Pyo Hong ${ }^{385}$, Jongwhi H. Hong ${ }^{394}$, Gerrit K. Hooijer ${ }^{395}$, Henrik Hornshøj ${ }^{73}$, Fumie Hosoda ${ }^{118}$, Yong Hou 56,396 , Volker Hovestadt 397 , William Howat ${ }^{352}$, Alan P. Hoyle ${ }^{134}$, Ralph H. Hruban ${ }^{149}$, Jianhong Hu ${ }^{12}$, Taobo Hu 64 , Xing Hua ${ }^{240}$, Kuan-lin Huang 1,398, Mei Huang ${ }^{176}$, Mi Ni Huang ${ }^{179,180}$, Vincent Huang ${ }^{6}$, Yi Huang ${ }^{65,66}$, Wolfgang Huber60, Thomas J. Hudson 272,399, Michael Hummel400, Jillian A. Hung246,247, David Huntsman 401, Ted R. Hupp 402 , Jason Huse 88 , Matthew R. Huska403, Barbara Hutter ${ }^{43,67,68}$, Carolyn M. Hutter ${ }^{27}$, Daniel Hübschmann 48,54,404,405,406, Christine A. lacobuzio-Donahue ${ }^{348}$, Charles David Imbusch ${ }^{205}$, Marcin Imielinski 407,408, Seiya Imoto 375 , William B. Isaacs ${ }^{409}$, Keren Isaev6,44, Shumpei Ishikawa ${ }^{410}$, Murat Iskar 397 , S. M. Ashiqul Islam 244, Michael Ittmann411,412,413, Sinisa Ivkovic ${ }^{284}$, Jose M. G. Izarzugaza ${ }^{414}$, Jocelyne Jacquemier ${ }^{415}$, Valerie Jakrot ${ }^{211}$, Nigel B. Jamieson 133,172,416, Gun Ho Jang ${ }^{148}$, Se Jin Jang ${ }^{417}$, Joy C. Jayaseelan ${ }^{12}$, Reyka Jayasinghe ${ }^{1}$, Stuart R. Jefferys ${ }^{134}$, Karine Jegalian ${ }^{418}$, Jennifer L. Jennings ${ }^{419}$, Seung-Hyup Jeon ${ }^{250}$, Lara Jerman 60,420, Yuan Ji ${ }^{421,422}$, Wei Jiao ${ }^{6}$, Peter A. Johansson ${ }^{311}$, Amber L. Johns ${ }^{170}$, Jeremy Johns ${ }^{272}$, Rory Johnson 230,423 , Todd A. Johnson ${ }^{183}$, Clemency Jolly 290 , Yann Joly ${ }^{424}$, Jon G. Jonasson ${ }^{320}$, Corbin D. Jones ${ }^{425}$, David R. Jones ${ }^{49}$, David T. W. Jones ${ }^{426,427}$, Nic Jones ${ }^{428}$, Steven J. M. Jones ${ }^{30}$, Jos Jonkers ${ }^{204}$, Young Seok Ju49,249, Hartmut Juhl429, Jongsun Jung69, Malene Juul ${ }^{73}$, Randi Istrup Juul ${ }^{73}$, Sissel Juul ${ }^{374}$, Natalie Jäger ${ }^{47}$, Rolf Kabbe ${ }^{47}$, Andre Kahles 276,277,278,279,430, Abdullah Kahraman 431,432,433, Vera B. Kaiser ${ }^{434}$, Hojabr Kakavand ${ }^{211}$, Sangeetha Kalimuthu148, Christof von Kalle ${ }^{405}$, Koo Jeong Kang 91 , Katalin Karaszi ${ }^{351}$, Beth Karlan ${ }^{435}$, Rosa Karlić436, Dennis Karsch ${ }^{437}$, Katayoon Kasaian ${ }^{29,30}$, Karin S. Kassahn 113,438, Hitoshi Katai ${ }^{439}$, Mamoru Kato ${ }^{440}$, Hiroto Katoh ${ }^{410}$, Yoshiiku Kawakami93, Jonathan D. Kay ${ }^{117}$, Stephen H. Kazakoff209,311, Marat D. Kazanov441,442,443, Maria Keays ${ }^{59}$, Electron Kebebew444,445, Richard F. Kefford ${ }^{446}$, Manolis Kellis ${ }^{8,447}$, James G. Kench ${ }^{170,350,448,}$ 
Catherine J. Kennedy 246,247, Jules N. A. Kerssemakers ${ }^{47}$, David Khoo ${ }^{273}$, Vincent Khoo ${ }^{221}$,

Narong Khuntikeo ${ }^{115,449}$, Ekta Khurana301,302,450,451, Helena Kilpinen ${ }^{117}$, Hark Kyun Kim ${ }^{452}$, Hyung-Lae Kim ${ }^{70}$, Hyung-Yong Kim ${ }^{415}$, Hyunghwan Kim ${ }^{250}$, Jaegil Kim ${ }^{8}$, Jihoon Kim ${ }^{453}$, Jong K. Kim ${ }^{454}$, Youngwook Kim ${ }^{455,456}$, Tari A. King 457,458,459, Wolfram Klapper ${ }^{128}$, Kortine Kleinheinz ${ }^{47,48}$, Leszek J. Klimczak ${ }^{460}$,

Stian Knappskog 49,461, Michael Kneba437, Bartha M. Knoppers ${ }^{424}$, Youngil Koh 462,463 , Jan Komorowski ${ }^{305,464}$, Daisuke Komura $^{410}$, Mitsuhiro Komura ${ }^{375}$, Gu Kong ${ }^{415}$, Marcel Kool ${ }^{426,465}$, Jan O. Korbel ${ }^{59,60}$, Viktoriya Korchina ${ }^{12}$, Andrey Korshunov ${ }^{465}$, Michael Koscher ${ }^{465}$, Roelof Koster ${ }^{466}$, Zsofia Kote-Jarai ${ }^{178,}$ Antonios Koures ${ }^{244}$, Milena Kovacevic ${ }^{284}$, Barbara Kremeyer ${ }^{49}$, Helene Kretzmer ${ }^{164,165}$, Markus Kreuz ${ }^{467}$, Savitri Krishnamurthy 99,468, Dieter Kube ${ }^{469}$, Kiran Kumar ${ }^{8}$, Pardeep Kumar ${ }^{221}$, Sushant Kumar ${ }^{5,19}$, Yogesh Kumar ${ }^{64}$, Ritika Kundra 114,121, Kirsten Kübler ${ }^{8,14,72}$, Ralf Küppers ${ }^{470}$, Jesper Lagergren ${ }^{383,471}$, Phillip H. Lai ${ }^{166}$, Peter W. Laird ${ }^{472}$, Sunil R. Lakhani ${ }^{473}$, Christopher M. Lalansingh ${ }^{6}$, Emilie Lalonde ${ }^{6}$, Fabien C. Lamaze ${ }^{6}$, Adam Lambert ${ }^{351}$, Eric Lander ${ }^{8}$, Pablo Landgraf ${ }^{474,475}$, Luca Landoni ${ }^{115}$, Anita Langerød ${ }^{130}$, Andrés Lanzós $230,231,423$, Denis Larsimont ${ }^{476}$, Erik Larsson ${ }^{477}$, Mark Lathrop ${ }^{189}$, Loretta M. S. Lau ${ }^{478}$, Chris Lawerenz ${ }^{55}$, Rita T. Lawlor ${ }^{177}$, Michael S. Lawrence ${ }^{8,72,183}$, Alexander J. Lazar 99,108, Xuan Le $^{479}$, Darlene Lee ${ }^{30}$, Donghoon Lee ${ }^{5}$, Eunjung Alice Lee ${ }^{480}$, Hee Jin Lee ${ }^{417}$, Jake June-Koo Lee 264,266, Jeong-Yeon Lee ${ }^{481}$, Juhee Lee ${ }^{482}$, Ming Ta Michael Lee ${ }^{340}$, Henry Lee-Six ${ }^{49}$, Kjong-Van Lehmann276,277,278,279,430, Hans Lehrach483, Dido Lenze ${ }^{400}$, Conrad R. Leonard 209,311 , Daniel A. Leongamornlert 49,178 , Ignaty Leshchiner ${ }^{8}$, Louis Letourneau ${ }^{484}$, Ivica Letunic ${ }^{71}$, Douglas A. Levine ${ }^{88,485}$, Lora Lewis ${ }^{12}$, Tim Ley ${ }^{486}$, Chang Li ${ }^{56,396}$, Constance H. Li $i^{6,44}$, Haiyan Irene $\mathrm{Li}^{30}$, Jun Li ${ }^{21}$, Lin Li ${ }^{56}$, Shantao Li ${ }^{5}$, Siliang Li ${ }^{56,396}$, Xiaobo Li ${ }^{56,396}$, Xiaotong Li ${ }^{5}$, Xinyue Li ${ }^{56}$, Yilong Li ${ }^{49}$, Han Liang ${ }^{21}$, Sheng-Ben Liang ${ }^{234}$, Peter Lichter 68,397, Pei Lin ${ }^{8}$, Ziao Lin ${ }^{8,487}$, W. M. Linehan ${ }^{488}$, Ole Christian Lingjærde ${ }^{489}$ Dongbing Liu 56,396, Eric Minwei Liu88,301,302, Fei-Fei Liu 201,490, Fenglin Liu381,491, Jia Liu ${ }^{492}$, Xingmin Liu 56,396, Julie Livingstone ${ }^{6}$, Dimitri Livitz ${ }^{8}$, Naomi Livni ${ }^{221}$, Lucas Lochovsky5,19,110, Markus Loeffler ${ }^{467}$, Georgina V. Long ${ }^{211}$, Armando Lopez-Guillermo493, Shaoke Lou ${ }^{5,19}$, David N. Louis ${ }^{72}$, Laurence B. Lovat ${ }^{117}$, Yiling Lu ${ }^{38}$, Yong-Jie Lu 162,494, Youyong Lu 495,496,497, Claudio Luchini ${ }^{167}$, Ilinca Lungu ${ }^{144,148}$, Xuemei Luo ${ }^{152}$, Hayley J. Luxton ${ }^{117}$, Andy G. Lynch223,315,498, Lisa Lype ${ }^{36}$, Cristina López ${ }^{111,112}$, Carlos López-Otín ${ }^{499}$, Eric Z. Ma ${ }^{64}$, Yussanne Ma ${ }^{30}$, Gaetan MacGrogan ${ }^{500}$, Shona MacRae ${ }^{501}$, Geoff Macintyre ${ }^{223}$, Tobias Madsen ${ }^{73}$, Kazuhiro Maejima ${ }^{184}$, Andrea Mafficini ${ }^{177}$, Dennis T. Maglinte 166,502 , Arindam Maitra ${ }^{174}$,

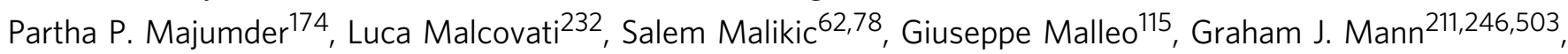
Luisa Mantovani-Löffler ${ }^{504}$, Kathleen Marchal505,506, Giovanni Marchegiani15, Elaine R. Mardis 1,193,507, Adam A. Margolin 31 , Maximillian G. Marin ${ }^{37}$, Florian Markowetz 223,315 , Julia Markowski ${ }^{403}$, Jeffrey Marks ${ }^{508}$, Tomas Marques-Bonet 61,81,386,509, Marco A. Marra ${ }^{30}$, Luke Marsden³1, John W. M. Martens ${ }^{332}$, Sancha Martin 49,510, Jose I. Martin-Subero 81,511, Iñigo Martincorena ${ }^{49}$, Alexander Martinez-Fundichely ${ }^{301,302,451}$ Yosef E. Maruvka 8,26,72, R. Jay Mashl ${ }^{1,2}$, Charlie E. Massie ${ }^{223}$, Thomas J. Matthew ${ }^{37}$, Lucy Matthews ${ }^{178}$, Erik Mayer 221,512, Simon Mayes ${ }^{513}$, Michael Mayo ${ }^{30}$, Faridah Mbabaali272, Karen McCune ${ }^{514}$, Ultan McDermott ${ }^{49}$, Patrick D. McGillivray ${ }^{19}$, Michael D. McLellan 1,3,20, John D. McPherson 148,272,515, John R. McPherson 179,180, Treasa A. McPherson ${ }^{260}$, Samuel R. Meier ${ }^{8}$, Alice Meng ${ }^{516}$, Shaowu Meng ${ }^{134}$, Andrew Menzies ${ }^{49}$, Neil D. Merrett ${ }^{115,517}$, Sue Merson ${ }^{178}$, Matthew Meyerson ${ }^{8,14,82}$, William U. Meyerson 4,5, Piotr A. Mieczkowski ${ }^{518}$, George L. Mihaiescu ${ }^{76}$, Sanja Mijalkovic ${ }^{284}$, Ana Mijalkovic Mijalkovic-Lazic ${ }^{284}$,

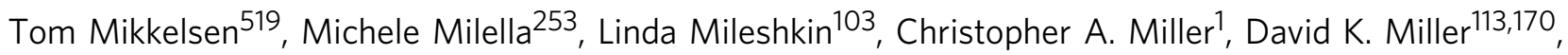
Jessica K. Miller ${ }^{272}$, Gordon B. Mills ${ }^{33,34}$, Ana Milovanovic ${ }^{57}$, Sarah Minner ${ }^{520}$, Marco Miotto ${ }^{115}$, Gisela Mir Arnau 267, Lisa Mirabello240, Chris Mitchell103, Thomas J. Mitchell 49,315,352, Satoru Miyano 375 , Naoki Miyoshi ${ }^{375}$, Shinichi Mizuno ${ }^{521}$, Fruzsina Molnár-Gábor ${ }^{522}$, Malcolm J. Moore ${ }^{300}$, Richard A. Moore ${ }^{30}$, 
Sandro Morganella ${ }^{49}$, Quaid D. Morris ${ }^{127,490}$, Carl Morrison ${ }^{523,524}$, Lisle E. Mose ${ }^{134}$, Catherine D. Moser ${ }^{349}$, Ferran Muiños 298,299, Loris Mularoni298,299, Andrew J. Mungall ${ }^{30}$, Karen Mungall ${ }^{30}$, Elizabeth A. Musgrove ${ }^{133}$, Ville Mustonen 525,526,527, David Mutch ${ }^{528}$, Francesc Muyas52,53,74, Donna M. Muzny ${ }^{12}$, Alfonso Muñoz ${ }^{59}$, Jerome Myers ${ }^{529}$, Ola Myklebost ${ }^{461}$, Peter Möller ${ }^{530}$, Genta Nagae ${ }^{89}$, Adnan M. Nagrial ${ }^{170}$, Hardeep K. Nahal-Bose ${ }^{76}$, Hitoshi Nakagama ${ }^{531}$, Hidewaki Nakagawa ${ }^{184}$, Hiromi Nakamura ${ }^{118}$, Toru Nakamura388, Kaoru Nakano ${ }^{184}$, Tannistha Nandi ${ }^{532}$, Jyoti Nangalia49, Mia Nastic ${ }^{284}$, Arcadi Navarro61,81,386, Fabio C. P. Navarro ${ }^{19}$, David E. Neal223,352, Gerd Nettekoven ${ }^{533}$, Felicity Newell ${ }^{209,311,}$ Steven J. Newhouse ${ }^{59}$, Yulia Newton ${ }^{37}$, Alvin Wei Tian Ng534, Anthony Ng535, Jonathan Nicholson ${ }^{49}$, David Nicol ${ }^{221}$, Yongzhan Nie ${ }^{321,536}$, G. Petur Nielsen ${ }^{72}$, Morten Muhlig Nielsen ${ }^{73}$, Serena Nik-Zainal ${ }^{49,281,282,537}$,

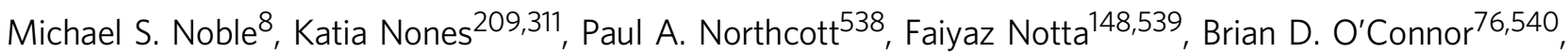
Peter O'Donnell ${ }^{541}$, Maria O'Donovan ${ }^{282}$, Sarah O'Meara ${ }^{49}$, Brian Patrick O'Neill ${ }^{542}$, J. Robert O'Neill ${ }^{543}$, David Ocana ${ }^{59}$, Angelica Ochoa ${ }^{88}$, Layla Oesper ${ }^{544}$, Christopher Ogden ${ }^{221}$, Hideki Ohdan ${ }^{93}$, Kazuhiro Ohi ${ }^{375}$, Lucila Ohno-Machado244, Karin A. Oien523,545, Akinyemi I. Ojesina546,547,548, Hidenori Ojima549, Takuji Okusaka550, Larsson Omberg551, Choon Kiat Ong552, Stephan Ossowski ${ }^{52,53,74}$, German Ott ${ }^{553}$, B. F. Francis Ouellette ${ }^{76,554}$, Christine P'ng6 ${ }^{6}$, Marta Paczkowska6 ${ }^{6}$ Salvatore Paiella15, Chawalit Pairojkul ${ }^{523}$, Marina Pajic ${ }^{170}$, Qiang Pan-Hammarström ${ }^{56,555}$, Elli Papaemmanuil ${ }^{49}$, Irene Papatheodorou ${ }^{59}$, Nagarajan Paramasivam 43,47, Ji Wan Park ${ }^{385}$, Joong-Won Park 556 , Keunchil Park ${ }^{557,558}$, Kiejung Park ${ }^{559}$, Peter J. Park 264,266 , Joel S. Parker ${ }^{518}$, Simon L. Parsons ${ }^{124}$, Harvey Pass ${ }^{560}$, Danielle Pasternack ${ }^{272}$, Alessandro Pastore 276, Ann-Marie Patch209,311, Iris Pauporté251, Antonio Pea ${ }^{115}$, John V. Pearson209,311, Chandra Sekhar Pedamallu 8,14,82, Jakob Skou Pedersen73,75, Paolo Pederzoli ${ }^{115}$, Martin Peifer ${ }^{270,}$ Nathan A. Pennell ${ }^{561}$, Charles M. Perou ${ }^{129,518}$, Marc D. Perry ${ }^{76,77}$, Gloria M. Petersen ${ }^{562}$, Myron Peto ${ }^{22}$, Nicholas Petrelli563, Robert Petryszak 59 , Stefan M. Pfister 426,465,564, Mark Phillips ${ }^{424}$, Oriol Pich 298,299, Hilda A. Pickett ${ }^{478}$, Todd D. Pih| ${ }^{565}$, Nischalan Pillay ${ }^{566}$, Sarah Pinder ${ }^{567}$, Mark Pinese ${ }^{170}$, Andreia V. Pinho ${ }^{568,}$ Esa Pitkänen ${ }^{60}$, Xavier Pivot ${ }^{569}$, Elena Piñeiro-Yáñez ${ }^{95}$, Laura Planko ${ }^{533}$, Christoph Plass ${ }^{345}$, Paz Polak ${ }^{8,14,15}$, Tirso Pons ${ }^{570}$, Irinel Popescu ${ }^{571}$, Olga Potapova ${ }^{572}$, Aparna Prasad ${ }^{52}$, Shaun R. Preston ${ }^{573}$, Manuel Prinz ${ }^{47}$, Antonia L. Pritchard ${ }^{311}$, Stephenie D. Prokopec ${ }^{6}$, Elena Provenzano 574 , Xose S. Puente ${ }^{499}$, Sonia Puig ${ }^{176}$, Montserrat Puiggròs ${ }^{57}$, Sergio Pulido-Tamayo505,506, Gulietta M. Pupo 246 , Colin A. Purdie ${ }^{575}$, Michael C. Quinn209,311, Raquel Rabionet52,53,576, Janet S. Rader 577, Bernhard Radlwimmer 397 , Petar Radovic ${ }^{284}$, Benjamin Raeder ${ }^{60}$, Keiran M. Raine ${ }^{49}$, Manasa Ramakrishna49, Kamna Ramakrishnan ${ }^{49}$, Suresh Ramalingam ${ }^{578}$, Benjamin J. Raphael ${ }^{579}$, W. Kimryn Rathmell ${ }^{580}$, Tobias Rausch ${ }^{60}$, Guido Reifenberger 475 , Jüri Reimand ${ }^{6,44}$, Jorge Reis-Filho ${ }^{348}$, Victor Reuter ${ }^{348}$, Iker Reyes-Salazar ${ }^{298}$, Matthew A. Reyna ${ }^{579}$, Sheila M. Reynolds ${ }^{36}$, Esther Rheinbay ${ }^{8,14,72}$, Yasser Riazalhosseini ${ }^{189}$, Andrea L. Richardson ${ }^{323}$, Julia Richter ${ }^{111,128}$, Matthew Ringel ${ }^{581}$, Markus Ringnér ${ }^{181}$, Yasushi Rino ${ }^{582}$, Karsten Rippe ${ }^{405}$, Jeffrey Roach ${ }^{583}$, Lewis R. Roberts ${ }^{349}$, Nicola D. Roberts ${ }^{49}$, Steven A. Roberts ${ }^{584}$, A. Gordon Robertson ${ }^{30}$, Alan J. Robertson ${ }^{113}$, Javier Bartolomé Rodriguez ${ }^{57}$,

Bernardo Rodriguez-Martin 104,105,106, F. Germán Rodríguez-González 83,332, Michael H. A. Roehrl44,123,148,234,585,586, Marius Rohde 587 , Hirofumi Rokutan ${ }^{440}$, Gilles Romieu ${ }^{588}$, Ilse Rooman ${ }^{170}$, Tom Roques ${ }^{262}$, Daniel Rosebrock ${ }^{8}$, Mara Rosenberg 8,72 , Philip C. Rosenstiel ${ }^{589}$, Andreas Rosenwald ${ }^{590}$, Edward W. Rowe ${ }^{221,591}$, Romina Royo ${ }^{57}$, Steven G. Rozen 179,180,592, Yulia Rubanova17,127, Mark A. Rubin 423,593,594,595,596, Carlota Rubio-Perez 298,299,597, Vasilisa A. Rudneva60, Borislav C. Rusev ${ }^{177}$, Andrea Ruzzenente ${ }^{598}$, Gunnar Rätsch276,277,278,279,280,430, Radhakrishnan Sabarinathan 298,299,599, Veronica Y. Sabelnykova ${ }^{6}$, Sara Sadeghi ${ }^{30}$, S. Cenk Sahinalp ${ }^{62,78,79}$, Natalie Saini ${ }^{357}$, Mihoko Saito-Adachi ${ }^{440}$, Gordon Saksena ${ }^{8}$, Adriana Salcedo ${ }^{6}$, Roberto Salgado ${ }^{600}$, Leonidas Salichos ${ }^{5,19}$, Richard Sallari ${ }^{8}$, Charles Saller ${ }^{601}$, Roberto Salvia ${ }^{115}$, Michelle Sam ${ }^{272}$, Jaswinder S. Samra115,602, Francisco Sanchez-Vega ${ }^{114,121}$, Chris Sander276,603,604, Grant Sanders ${ }^{134}$, Rajiv Sarin ${ }^{605}$, 
Iman Sarrafi62,78, Aya Sasaki-Oku ${ }^{184}$, Torill Sauer ${ }^{489}$, Guido Sauter ${ }^{520}$, Robyn P. M. Saw ${ }^{211}$, Maria Scardoni ${ }^{167}$, Christopher J. Scarlett ${ }^{170,606}$, Aldo Scarpa ${ }^{177}$, Ghislaine Scelo ${ }^{194}$, Dirk Schadendorf ${ }^{68,607}$, Jacqueline E. Schein ${ }^{30}$, Markus B. Schilhabel ${ }^{589}$, Matthias Schlesner ${ }^{47,80}$, Thorsten Schlomm ${ }^{84,608}$, Heather K. Schmidt ${ }^{1}$, Sarah-Jane Schramm ${ }^{246}$, Stefan Schreiber ${ }^{609}$, Nikolaus Schultz ${ }^{121}$, Steven E. Schumacher ${ }^{8,323}$, Roland F. Schwarz 59,403,405,610, Richard A. Scolyer 211,448,602, David Scott ${ }^{428}$, Ralph Scully611, Raja Seethala612, Ayellet V. Segre ${ }^{8,613}$, Iris Selander ${ }^{260}$, Colin A. Semple ${ }^{434}$, Yasin Senbabaoglu ${ }^{276}$, Subhajit Sengupta ${ }^{614}$, Elisabetta Sereni ${ }^{115}$, Stefano Serra ${ }^{585}$, Dennis C. Sgroi ${ }^{72}$, Mark Shackleton ${ }^{103}$, Nimish C. Shah ${ }^{352}$, Sagedeh Shahabi ${ }^{234}$, Catherine A. Shang ${ }^{329}$, Ping Shang 211 , Ofer Shapira ${ }^{8,323}$, Troy Shelton ${ }^{271}$, Ciyue Shen ${ }^{603,604}$, Hui Shen ${ }^{615}$, Rebecca Shepherd ${ }^{49}$, Ruian Shi ${ }^{490}$, Yan Shi ${ }^{134}$, Yu-Jia Shiah ${ }^{6}$, Tatsuhiro Shibata ${ }^{118,616}$, Juliann Shih ${ }^{8,82}$, Eigo Shimizu ${ }^{375}$, Kiyo Shimizu ${ }^{617}$, Seung Jun Shin ${ }^{618}$, Yuichi Shiraishi ${ }^{375}$, Tal Shmaya ${ }^{285}$, Ilya Shmulevich ${ }^{36}$, Solomon I. Shorser ${ }^{6}$, Charles Short ${ }^{59}$, Raunak Shrestha ${ }^{62}$, Suyash S. Shringarpure ${ }^{217}$, Craig Shriver ${ }^{619}$, Shimin Shuai ${ }^{6,126}$, Nikos Sidiropoulos ${ }^{83}$, Reiner Siebert ${ }^{112,620}$, Anieta M. Sieuwerts ${ }^{332}$, Lina Sieverling 205,237 , Sabina Signoretti202,621, Katarzyna O. Sikora ${ }^{177}$, Michele Simbolo ${ }^{138}$, Ronald Simon ${ }^{520}$, Janae V. Simons ${ }^{134}$, Jared T. Simpson 6,17, Peter T. Simpson 473, Samuel Singer ${ }^{115,458}$, Nasa Sinnott-Armstrong 8,217, Payal Sipahimalani ${ }^{30}$, Tara J. Skelly ${ }^{390}$, Marcel Smid ${ }^{332}$, Jaclyn Smith ${ }^{622}$, Karen Smith-McCune ${ }^{514}$, Nicholas D. Socci ${ }^{276}$, Heidi J. Sofia ${ }^{27}$, Matthew G. Soloway ${ }^{134}$, Lei Song 240 , Anil K. Sood $623,624,625$, Sharmila Sothi ${ }^{626}$, Christos Sotiriou ${ }^{244}$, Cameron M. Soulette ${ }^{37}$, Paul N. Span ${ }^{627}$, Paul T. Spellman ${ }^{22}$, Nicola Sperandio ${ }^{177}$, Andrew J. Spillane ${ }^{211}$, Oliver Spiro ${ }^{8}$, Jonathan Spring ${ }^{628}$, Johan Staaf ${ }^{181}$, Peter F. Stadler ${ }^{163,164,165}$, Peter Staib ${ }^{629}$, Stefan G. Stark $277,279,618,630$, Lucy Stebbings ${ }^{49}$, Ólafur Andri Stefánsson ${ }^{631}$, Oliver Stegle 59,60,632, Lincoln D. Stein ${ }^{6,126}$, Alasdair Stenhouse ${ }^{633}$, Chip Stewart ${ }^{8}$, Stephan Stilgenbauer ${ }^{634}$, Miranda D. Stobbe ${ }^{52,61}$, Michael R. Stratton 49, Jonathan R. Stretch ${ }^{211}$, Adam J. Struck ${ }^{31}$, Joshua M. Stuart ${ }^{24,37}$, Henk G. Stunnenberg 396,635, Hong Su ${ }^{56,396}$, Xiaoping Su99, Ren X. Sun ${ }^{6}$, Stephanie Sungalee ${ }^{60}$, Hana Susak ${ }^{52,53}$, Akihiro Suzuki ${ }^{89,636}$, Fred Sweep ${ }^{637}$, Monika Szczepanowski ${ }^{128}$, Holger Sültmann 67,638, Takashi Yugawa617, Angela Tam ${ }^{30}$, David Tamborero 298,299, Benita Kiat Tee Tan ${ }^{639}$, Donghui Tan ${ }^{518}$, Patrick Tan 180,532,592,640, Hiroko Tanaka375, Hirokazu Taniguchi616, Tomas J. Tanskanen ${ }^{641}$, Maxime Tarabichi ${ }^{49,290}$, Roy Tarnuzzer ${ }^{220}$, Patrick Tarpey ${ }^{642}$, Morgan L. Taschuk ${ }^{152}$, Kenji Tatsuno ${ }^{89}$, Simon Tavaré223,643, Darrin F. Taylor ${ }^{113}$, Amaro Taylor-Weiner ${ }^{8}$, Jon W. Teague ${ }^{49}$, Bin Tean Teh ${ }^{180,592,640,644,645}$, Varsha Tembe ${ }^{246}$, Javier Temes ${ }^{104,105}$, Kevin Thai ${ }^{76}$, Sarah P. Thayer ${ }^{393}$, Nina Thiessen ${ }^{30}$, Gilles Thomas ${ }^{646}$, Sarah Thomas ${ }^{221}$, Alan Thompson ${ }^{221}$,

Alastair M. Thompson ${ }^{633}$, John F. Thompson ${ }^{211}$, R. Houston Thompson ${ }^{647}$, Heather Thorne ${ }^{103}$, Leigh B. Thorne ${ }^{176}$, Adrian Thorogood ${ }^{424}$, Grace Tiao ${ }^{8}$, Nebojsa Tijanic ${ }^{284}$, Lee E. Timms ${ }^{272}$, Roberto Tirabosco648, Marta Tojo ${ }^{106}$, Stefania Tommasi ${ }^{649}$, Christopher W. Toon ${ }^{170}$, Umut H. Toprak ${ }^{48,650}$, David Torrents ${ }^{57,81}$, Giampaolo Tortora 651,652 , Jörg Tost ${ }^{653}$, Yasushi Totoki ${ }^{118}$, David Townend ${ }^{654}$, Nadia Traficante ${ }^{103}$, Isabelle Treilleux 655,656, Jean-Rémi Trotta61, Lorenz H. P. Trümper ${ }^{469}$, Ming Tsao ${ }^{124,539}$, Tatsuhiko Tsunoda183,657,658,659, Jose M. C. Tubio 104,105,106, Olga Tucker ${ }^{660}$, Richard Turkington ${ }^{661}$, Daniel J. Turner ${ }^{513}$, Andrew Tutt ${ }^{323}$, Masaki Ueno ${ }^{376}$, Naoto T. Ueno662, Christopher Umbricht ${ }^{151,213,663,}$ Husen M. Umer ${ }^{305,664}$, Timothy J. Underwood ${ }^{665}$, Lara Urban 59,60 , Tomoko Urushidate ${ }^{616}$, Tetsuo Ushiku ${ }^{339}$, Liis Uusküla-Reimand 666,667, Alfonso Valencia57,81, David J. Van Den Berg ${ }^{166}$, Steven Van Laere ${ }^{307}$, Peter Van Loo 290,291 , Erwin G. Van Meir 668 , Gert G. Van den Eynden ${ }^{307}$, Theodorus Van der Kwast ${ }^{123}$, Naveen Vasudev ${ }^{137}$, Miguel Vazquez ${ }^{57,669}$, Ravikiran Vedururu ${ }^{267}$, Umadevi Veluvolu ${ }^{518}$, Shankar Vembu 490,670, Lieven P. C. Verbeke 506,671, Peter Vermeulen 307 , Clare Verrill ${ }^{351,672}$, Alain Viari ${ }^{177}$, David Vicente ${ }^{57}$, Caterina Vicentini ${ }^{177}$, K. Vijay Raghavan ${ }^{365}$, Juris Viksna673, Ricardo E. Vilain ${ }^{674}$, Izar Villasante ${ }^{57,}$ Anne Vincent-Salomon ${ }^{635}$, Tapio Visakorpi ${ }^{190}$, Douglas Voet ${ }^{8}$, Paresh Vyas ${ }^{311,351,}$ Ignacio Vázquez-García49,86,675,676, Nick M. Waddell209, Nicola Waddell209,311, Claes Wadelius677, Lina Wadi6, 
Rabea Wagener ${ }^{111,112}$, Jeremiah A. Wala ${ }^{8,14,82}$, Jian Wang56, Jiayin Wang1,40,66, Linghua Wang ${ }^{12}$, Qi Wang ${ }^{465}$, Wenyi Wang ${ }^{21}$, Yumeng Wang ${ }^{21}$, Zhining Wang 220 , Paul M. Waring ${ }^{523}$, Hans-Jörg Warnatz ${ }^{483}$, Jonathan Warrell ${ }^{5,19}$, Anne Y. Warren ${ }^{352,678}$, Sebastian M. Waszak ${ }^{60}$, David C. Wedge 49,294,679, Dieter Weichenhan ${ }^{345}$, Paul Weinberger ${ }^{680}$, John N. Weinstein ${ }^{38}$, Joachim Weischenfeldt $60,83,84$, Daniel J. Weisenberger ${ }^{166}$, lan Welch ${ }^{681}$, Michael C. Wend1 1,10,11, Johannes Werner ${ }^{47,85}$, Justin P. Whalley ${ }^{61,682}$, David A. Wheeler ${ }^{12,13}$, Hayley C. Whitaker ${ }^{117}$, Dennis Wigle ${ }^{683}$, Matthew D. Wilkerson ${ }^{518}$, Ashley Williams ${ }^{244}$, James S. Wilmott ${ }^{211}$, Gavin W. Wilson ${ }^{6,148}$, Julie M. Wilson ${ }^{148}$, Richard K. Wilson ${ }^{1,684}$, Boris Winterhoff ${ }^{685}$, Jeffrey A. Wintersinger ${ }^{17,127,384}$, Maciej Wiznerowicz ${ }^{686,687}$, Stephan Wolf ${ }^{688}$, Bernice H. Wong689, Tina Wong ${ }^{1,30}$, Winghing Wong ${ }^{690}$, Youngchoon Woo ${ }^{250}$, Scott Wood ${ }^{209,311}$, Bradly G. Wouters ${ }^{44}$, Adam J. Wright ${ }^{6}$, Derek W. Wright ${ }^{133,691}$, Mark H. Wright ${ }^{217}$, Chin-Lee Wu ${ }^{72}$, Dai-Ying Wu ${ }^{285}$, Guanming Wu ${ }^{692}$, Jianmin Wu ${ }^{170}$, Kui Wu $u^{56,396}$, Yang Wu ${ }^{179,180}$, Zhenggang Wu${ }^{64}$, Liu Xi², Tian Xia693, Qian Xiang ${ }^{76}$, Xiao Xiao66, Rui Xing ${ }^{497}$, Heng Xiong56,396, Qinying Xu209,311, Yanxun Xu694, Hong Xue ${ }^{64}$, Shinichi Yachida118,695, Sergei Yakneen ${ }^{60}$, Rui Yamaguchi ${ }^{375}$, Takafumi N. Yamaguchi6, Masakazu Yamamoto ${ }^{120}$, Shogo Yamamoto 89 , Hiroki Yamaue ${ }^{376,}$ Fan Yang 490 , Huanming Yang ${ }^{56}$, Jean Y. Yang ${ }^{696}$, Liming Yang 220 , Lixing Yang ${ }^{697}$, Shanlin Yang ${ }^{306}$, Tsun-Po Yang 270 , Yang Yang ${ }^{369}$, Xiaotong Yao 408,698, Marie-Laure Yaspo ${ }^{483}$, Lucy Yates ${ }^{49}$, Christina Yau ${ }^{156}$, Chen $Y e^{56,396}$, Kai Ye 40,41, Venkata D. Yellapantula ${ }^{20,86}$, Christopher J. Yoon ${ }^{249}$, Sung-Soo Yoon ${ }^{463}$, Fouad Yousif6, Jun Yu ${ }^{699}$, Kaixian Yu ${ }^{700}$, Willie Yu ${ }^{701}$, Yingyan Yu ${ }^{702}$, Ke Yuan223,510,703, Yuan Yuan ${ }^{21}$, Denis Yuen ${ }^{6}$, Takashi Yugawa617, Christina K. Yung ${ }^{76}$, Olga Zaikova704, Jorge Zamora49,104,105,106, Marc Zapatka397, Jean C. Zenklusen ${ }^{220}$, Thorsten Zenz ${ }^{67}$, Nikolajs Zeps ${ }^{705,706}$, Cheng-Zhong Zhang ${ }^{8,707}$, Fan Zhang ${ }^{381}$, Hailei Zhang ${ }^{8}$, Hongwei Zhang ${ }^{494}$, Hongxin Zhang ${ }^{121}$, Jiashan Zhang ${ }^{220}$, Jing Zhang ${ }^{5}$, Junjun Zhang ${ }^{76}$, Xiuqing Zhang ${ }^{56}$, Xuanping Zhang66,369, Yan Zhang ${ }^{5,708,709}$, Zemin Zhang ${ }^{381,710}$, Zhongming Zhao ${ }^{711}$, Liangtao Zheng ${ }^{381}$, Xiuqing Zheng ${ }^{381}$, Wanding Zhou ${ }^{615}$, Yong Zhou ${ }^{56}$, Bin Zhu ${ }^{240}$, Hongtu Zhu ${ }^{700,712,}$ Jingchun Zhu ${ }^{24}$, Shida Zhu ${ }^{56,396}$, Lihua Zou ${ }^{713}$, Xueqing Zou ${ }^{49}$, Anna deFazio 246,247,714, Nicholas van As ${ }^{221}$, Carolien H. M. van Deurzen ${ }^{715}$, Marc J. van de Vijver ${ }^{523}$, L. van't Veer ${ }^{716}$ \& Christian von Mering 433,717

\footnotetext{
${ }^{87}$ Applied Tumor Genomics Research Program, Research Programs Unit, University of Helsinki, 00100 Helsinki, Finland. ${ }^{88}$ Memorial Sloan Kettering Cancer Center, New York, NY 10065, USA. ${ }^{89}$ Genome Science Division, Research Center for Advanced Science and Technology, University of Tokyo, Tokyo 113-8654, Japan. ${ }^{90}$ Department of Surgery, University of Chicago, Chicago, IL 60637, USA. ${ }^{91}$ Department of Surgery, Division of Hepatobiliary and Pancreatic Surgery, School of Medicine, Keimyung University Dongsan Medical Center, Daegu, South Korea. ${ }^{92}$ Department of Oncology, Gil Medical Center, Gachon University, Incheon, South Korea. ${ }^{93}$ Hiroshima University, Hiroshima 739-8511, Japan. ${ }^{94}$ King Faisal Specialist Hospital and Research Centre, Al Maather, Riyadh, Saudi Arabia. ${ }^{95}$ Bioinformatics Unit, Spanish National Cancer Research Centre (CNIO), 28029 Madrid, Spain. ${ }^{96}$ Bioinformatics Core Facility, University Medical Center Hamburg, 20251 Hamburg, Germany. ${ }^{97}$ Heinrich Pette Institute, Leibniz Institute for Experimental Virology, 20251 Hamburg, Germany. ${ }^{98}$ Ontario Tumour Bank, Ontario Institute for Cancer Research, Toronto, ON M5G OA3, Canada. ${ }^{99}$ Department of Pathology, The University of Texas MD Anderson Cancer Center, Houston, TX 77030, USA. ${ }^{100}$ Laboratory of Pathology, Center for Cancer Research, National Cancer Institute, Bethesda, MD 20814, USA. ${ }^{101}$ Department of Cellular and Molecular Medicine and Department of Bioengineering, University of California San Diego, La Jolla, CA 92093, USA. ${ }^{102}$ UC San Diego Moores Cancer Center, San Diego, CA 92037, USA. ${ }^{103}$ Sir Peter MacCallum Department of Oncology, Peter MacCallum Cancer Centre, University of Melbourne, Melbourne, VIC 3010, Australia. ${ }^{104}$ Centre for Research in Molecular Medicine and Chronic Diseases (CiMUS), Universidade de Santiago de Compostela, 15705 Santiago de Compostela, Spain. ${ }^{105}$ Department of Zoology, Genetics and Physical Anthropology, (CiMUS), Universidade de Santiago de Compostela, 15705 Santiago de Compostela, Spain. ${ }^{106}$ The Biomedical Research Centre (CINBIO), Universidade de Vigo, 36310 Vigo, Spain. ${ }^{107}$ Royal National Orthopaedic Hospital-Bolsover, London 36310, UK. ${ }^{108}$ Department of Genomic Medicine, The University of Texas MD Anderson Cancer Center, Houston, TX 77030, USA. ${ }^{109}$ Quantitative and Computational Biosciences Graduate Program, Baylor College of Medicine, Houston, TX 77030, USA. ${ }^{110}$ The Jackson Laboratory for Genomic Medicine, Farmington, CT 06032, USA. ${ }^{111}$ Institute of Human Genetics, Christian-Albrechts-University, 24118 Kiel, Germany. ${ }^{112}$ Institute of Human Genetics, Ulm University and Ulm University Medical Center, 89081 Ulm, Germany. ${ }^{113}$ Queensland Centre for Medical Genomics, Institute for Molecular Bioscience, University of Queensland, St. Lucia, Brisbane, QLD 4072, Australia. ${ }^{114}$ Salford Royal NHS Foundation Trust, Salford M6 8HD, UK. ${ }^{115}$ Department of Surgery, Pancreas Institute, University and Hospital Trust of Verona, 37129 Verona, Italy. ${ }^{116}$ Department of Molecular Oncology, BC Cancer Research Centre, Vancouver, BC V5Z 1L3, Canada. ${ }^{117}$ University College London, London WC1E 6BT, UK. ${ }^{118}$ Division of Cancer Genomics, National Cancer Center Research Institute, National Cancer Center, Tokyo 104-0045, Japan. ${ }^{119}$ DLR Project Management Agency, Bonn, Germany. ${ }^{120}$ Tokyo Women's Medical University, Tokyo 162-8666, Japan. ${ }^{121}$ Center for Molecular Oncology, Memorial Sloan Kettering Cancer Center, New York, NY 10065 , USA. ${ }^{122}$ Los Alamos National Laboratory, Los Alamos, NM 87545, USA. ${ }^{123}$ Department of Pathology, University Health Network, Toronto General Hospital, Toronto, ON M5G 2C4, Canada. ${ }^{124}$ Nottingham University Hospitals NHS Trust, Nottingham NG5 1PB, UK. ${ }^{125}$ Epigenomics and Cancer Risk Factors, German Cancer Research Center (DKFZ), 69120 Heidelberg, Germany. ${ }^{126}$ Department of Molecular Genetics, University of Toronto, Toronto, ON M5S, Canada. ${ }^{127}$ Vector Institute, Toronto, ON M5G 1M1, Canada. ${ }^{128}$ Hematopathology Section, Institute of Pathology,
} 
Christian-Albrechts-University, Kiel 24118, Germany. ${ }^{129}$ Department of Pathology and Laboratory Medicine, School of Medicine, University of North Carolina at Chapel Hill, Chapel Hill, NC 27599, USA. ${ }^{130}$ Department of Cancer Genetics, Institute for Cancer Research, Oslo University Hospital, The Norwegian Radium Hospital, 0450 Oslo, Norway. ${ }^{131}$ Pathology, Hospital Clinic, Institut d'Investigacions Biomèdiques August Pi i Sunyer (IDIBAPS), University of Barcelona, 08007 Barcelona, Spain. ${ }^{132}$ Department of Veterinary Medicine, Transmissible Cancer Group, University of Cambridge, Cambridge CB2 1TN, UK. ${ }^{133}$ Wolfson Wohl Cancer Research Centre, Institute of Cancer Sciences, University of Glasgow, Glasgow G12 8QQ, UK. ${ }^{134}$ Lineberger Comprehensive Cancer Center, University of North Carolina at Chapel Hill, Chapel Hill, NC 27599 , USA. ${ }^{135}$ Dana-Farber/Boston Children's Cancer and Blood Disorders Center, Boston, MA 02115, USA. ${ }^{136}$ Department of Pediatrics, Harvard Medical School, Boston, MA 02115, USA. ${ }^{137}$ Leeds Institute of Medical Research at St. James's, University of Leeds, St. James's University Hospital, Leeds LS2 9JT, UK. ${ }^{138}$ Department of Pathology and Diagnostics, University and Hospital Trust of Verona, 37129 Verona, Italy. ${ }^{139}$ Department of Surgery, Princess Alexandra Hospital, Brisbane, QLD 4102, Australia. ${ }^{140}$ Surgical Oncology Group, Diamantina Institute, University of Queensland, Brisbane, QLD 4072, Australia. ${ }^{141}$ Department of Population and Quantitative Health Sciences, Case Western Reserve University School of Medicine, Cleveland, $\mathrm{OH} 44106, \mathrm{USA} .{ }^{142}$ Research Health Analytics and Informatics, University Hospitals Cleveland Medical Center, Cleveland, OH 44106, USA. ${ }^{143}$ Gloucester Royal Hospital, Gloucester GL1 3NN, UK. ${ }^{144}$ Diagnostic Development, Ontario Institute for Cancer Research, Toronto, ON M5G OA3, Canada. ${ }^{145}$ Arnie Charbonneau Cancer Institute, University of Calgary, Calgary, AB T2N 1N4, Canada. ${ }^{146}$ Departments of Surgery and Oncology, University of Calgary, Calgary, AB T2N 1N4, Canada. ${ }^{147}$ Department of Pathology, Oslo University Hospital, The Norwegian Radium Hospital, Oslo, Norway. ${ }^{148}$ PanCuRx Translational Research Initiative, Ontario Institute for Cancer Research, Toronto, ON M5G OA3, Canada. ${ }^{149}$ Department of Oncology, Sidney Kimmel Comprehensive Cancer Center at Johns Hopkins University School of Medicine, Baltimore, MD 21231, USA. ${ }^{150}$ University Hospital Southampton NHS Foundation Trust, Southampton SO16 6YD, UK. ${ }^{151}$ Royal Stoke University Hospital, Stoke-on-Trent ST4 6QG, UK. ${ }^{152}$ Genome Sequence Informatics, Ontario Institute for Cancer Research, Toronto, ON M5G OA3, Canada. ${ }^{153}$ Human Longevity Inc, San Diego, CA M5G 0A3, USA. ${ }^{154}$ Olivia Newton-John Cancer Research Institute, La Trobe University, Heidelberg, VIC 3086, Australia. ${ }^{155}$ Genome Canada, Ottawa, ON K2P 1P1, Canada. ${ }^{156}$ Buck Institute for Research on Aging, Novato, CA 94945, USA. ${ }^{157}$ Duke University Medical Center, Durham, NC 27710, USA. ${ }^{158}$ Department of Human Genetics, Hannover Medical School, 30625 Hannover, Germany. ${ }^{159}$ Center for Bioinformatics and Functional Genomics, Cedars-Sinai Medical Center, Los Angeles, CA 90048, USA. ${ }^{160}$ Department of Biomedical Sciences, Cedars-Sinai Medical Center, Los Angeles, CA 90048, USA. ${ }^{161}$ The Hebrew University Faculty of Medicine, Jerusalem 9112102, Israel. ${ }^{162}$ Barts Cancer Institute, Barts and the London School of Medicine and Dentistry, Queen Mary University of London, London E1 4NS, UK. ${ }^{163}$ Department of Computer Science, Bioinformatics Group, University of Leipzig, 04109 Leipzig, Germany. ${ }^{164}$ Interdisciplinary Center for Bioinformatics, University of Leipzig, 04109 Leipzig, Germany. ${ }^{165}$ Transcriptome Bioinformatics, LIFE Research Center for Civilization Diseases, University of Leipzig, 04109 Leipzig, Germany. ${ }^{166}$ USC Norris Comprehensive Cancer Center, University of Southern California, Los Angeles, CA 90007, USA. ${ }^{167}$ Department of Diagnostics and Public Health, University and Hospital Trust of Verona, Verona 37129, Italy. ${ }^{168}$ Department of Mathematics, Aarhus University, 8000 Aarhus, Denmark. ${ }^{169}$ Instituto Carlos Slim de la Salud, Mexico City, Mexico. ${ }^{170}$ Cancer Division, Garvan Institute of Medical Research, Kinghorn Cancer Centre, University of New South Wales (UNSW Sydney), Sydney, NSW 2052, Australia. ${ }^{171}$ South Western Sydney Clinical School, Faculty of Medicine, University of New South Wales (UNSW Sydney), Liverpool, NSW 2052, Australia. ${ }^{172}$ West of Scotland Pancreatic Unit, Glasgow Royal Infirmary, Glasgow G4 OSF, UK. ${ }^{173}$ The Preston Robert Tisch Brain Tumor Center, Duke University Medical Center, Durham, NC 27710, USA. ${ }^{174}$ National Institute of Biomedical Genomics, Kalyani, West Bengal 741251, India. ${ }^{175}$ Institute of Clinical Medicine and Institute of Oral Biology, University of Oslo, 0315 Oslo, Norway. ${ }^{176}$ University of North Carolina at Chapel Hill, Chapel Hill, NC 27599 , USA. ${ }^{177}$ ARC-Net Centre for Applied Research on Cancer, University and Hospital Trust of Verona, Verona 0315, Italy. ${ }^{178}$ The Institute of Cancer Research, London SM2 $5 N G$, UK ${ }^{179}$ Centre for Computational Biology, Duke-NUS Medical School, Singapore 169857, Singapore. ${ }^{180}$ Programme in Cancer and Stem Cell Biology, Duke-NUS Medical School, Singapore 169857, Singapore. ${ }^{181}$ Division of Oncology and Pathology, Department of Clinical Sciences Lund, Lund University, Lund, Sweden. ${ }^{182}$ Department of Pediatric Oncology, Hematology and Clinical Immunology, Heinrich-Heine-University, 40225 Düsseldorf, Germany. ${ }^{183}$ Laboratory for Medical Science Mathematics, RIKEN Center for Integrative Medical Sciences, Yokohama, Japan. ${ }^{184}$ RIKEN Center for Integrative Medical Sciences, Yokohama, Japan. ${ }^{185}$ Department of Internal Medicine/Hematology, Friedrich-Ebert-Hospital, Neumünster, Germany. ${ }^{186}$ Departments of Dermatology and Pathology, Yale University, New Haven, CT 06520, USA. ${ }^{187}$ Radcliffe Department of Medicine, University of Oxford, Oxford OX1 2JD, UK. ${ }^{188}$ Canadian Center for Computational Genomics, McGill University, Montreal, QC H3A OG4, Canada. ${ }^{189}$ Department of Human Genetics, McGill University, Montreal, QC H3A OG4, Canada. ${ }^{190}$ Faculty of Medicine and Health Technology, Tampere University and Tays Cancer Center, Tampere University Hospital, 33520 Tampere, Finland. ${ }^{191}$ Haematology, Leeds Teaching Hospitals NHS Trust, Leeds LS1 3EX, UK. ${ }^{192}$ Translational Research and Innovation, Centre Léon Bérard, Lyon, France. ${ }^{193}$ Fox Chase Cancer Center, Philadelphia, PA 19111, USA. ${ }^{194}$ International Agency for Research on Cancer, World Health Organization, Lyon, France. ${ }^{195}$ Earlham Institute, Norwich NR4 7 UZ, UK. ${ }^{196}$ Norwich Medical School, University of East Anglia, Norwich NR4 7TJ, UK. ${ }^{197}$ Department of Molecular Biology, Faculty of Science, Radboud Institute for Molecular Life Sciences, Radboud University, Nijmegen, HB 6525 XZ, The Netherlands. ${ }^{198}$ CRUK Manchester Institute and Centre, Manchester M20 4GJ, UK. ${ }^{199}$ Department of Radiation Oncology, University of Toronto, Toronto, ON M5S, Canada. ${ }^{200}$ Division of Cancer Sciences, Manchester Cancer Research Centre, University of Manchester, Manchester M13 9PL, UK. ${ }^{201}$ Radiation Medicine Program, Princess Margaret Cancer Centre, Toronto, ON M5G 2C1, Canada. ${ }^{202}$ Department of Pathology, Brigham and Women's Hospital, Harvard Medical School, Boston, MA 02115, USA. ${ }^{203}$ Department of Surgery, Division of Thoracic Surgery, The Johns Hopkins University School of Medicine, Baltimore, MD 21205, USA. ${ }^{204}$ Division of Molecular Pathology, The Netherlands Cancer Institute, Oncode Institute, Amsterdam, CX 1066, The Netherlands. ${ }^{205}$ Division of Applied Bioinformatics, German Cancer Research Center (DKFZ), 69120 Heidelberg, Germany. ${ }^{206}$ German Cancer Genome Consortium (DKTK), Heidelberg, Germany. ${ }^{207}$ Center for Biological Sequence Analysis, Department of Bio and Health Informatics, Technical University of Denmark, 2800 Lyngby, Denmark. ${ }^{208}$ Novo Nordisk Foundation Center for Protein Research, University of Copenhagen, Copenhagen 1165 , Denmark. ${ }^{209}$ Institute for Molecular Bioscience, University of Queensland, St. Lucia, Brisbane, QLD 4072, Australia. ${ }^{210}$ Federal Ministry of Education and Research, Berlin, Germany. ${ }^{211}$ Melanoma Institute Australia, University of Sydney, Sydney, NSW 2006, Australia. ${ }^{212}$ Pediatric Hematology and Oncology, University Hospital Muenster, 48149 Muenster, Germany. ${ }^{213}$ Department of Pathology, Johns Hopkins University School of Medicine, Baltimore, MD 21205, USA. ${ }^{214}$ McKusick-Nathans Institute of Genetic Medicine, Sidney Kimmel Comprehensive Cancer Center at Johns Hopkins University School of Medicine, Baltimore, MD 21231, USA. ${ }^{215}$ Foundation Medicine, Inc, Cambridge, MA 02141, USA. ${ }^{216}$ Department of Biomedical Data Science, Stanford University School of Medicine, Stanford, CA 94305, USA. ${ }^{217}$ Department of Genetics, Stanford University School of Medicine, Stanford, CA 94305, USA. ${ }^{218}$ Bakar Computational Health Sciences Institute and Department of Pediatrics, University of California, San Francisco, CA 94110, USA. ${ }^{219}$ Institute of Clinical Medicine, Faculty of Medicine, University of Oslo, Oslo 0315, Norway. ${ }^{220}$ National Cancer Institute, National Institutes of Health, Bethesda, MD 20892, USA. ${ }^{221}$ Royal Marsden NHS Foundation Trust, London and Sutton, Sutton SM2 5PT, UK. ${ }^{222}$ Department of Oncology, University of Cambridge, Cambridge CB2 1TN, UK. ${ }^{223}$ Li Ka Shing Centre, Cancer Research UK Cambridge Institute, University of Cambridge, Cambridge CB2 1TN, UK. ${ }^{224}$ Institut Gustave Roussy, 94800 Villejuif, France. ${ }^{225}$ Department of Haematology, University of Cambridge, Cambridge CB2 1TN, UK. ${ }^{226}$ Anatomia Patológica, Hospital Clinic, Institut d'Investigacions Biomèdiques August Pi i Sunyer (IDIBAPS), University of Barcelona, 
08007 Barcelona, Spain. ${ }^{227}$ Spanish Ministry of Science and Innovation, Madrid, Spain. ${ }^{228}$ University of Michigan Comprehensive Cancer Center, Ann Arbor, MI 48109, USA. ${ }^{229}$ Department for BioMedical Research, University of Bern, 3012 Bern, Switzerland. ${ }^{230}$ Department of Medical Oncology, Inselspital, University Hospital and University of Bern, 3012 Bern, Switzerland. ${ }^{231}$ Graduate School for Cellular and Biomedical Sciences, University of Bern, 3012 Bern, Switzerland. ${ }^{232}$ University of Pavia, 27100 Pavia, Italy. ${ }^{233}$ University of Alabama at Birmingham, Birmingham, AL 35294, USA. ${ }^{234}$ UHN Program in BioSpecimen Sciences, Toronto General Hospital, Toronto, ON M5G 2C4, Canada. ${ }^{235}$ Department of Urology, Icahn School of Medicine at Mount Sinai, New York, NY 10029, USA. ${ }^{236}$ Centre for Law and Genetics, University of Tasmania, Sandy Bay Campus, Hobart, TAS 7005, Australia. ${ }^{237}$ Faculty of Biosciences, Heidelberg University, 69117 Heidelberg, Germany. ${ }^{238}$ Department of Biochemistry, Microbiology and Immunology, Faculty of Medicine, University of Ottawa, Ottawa, ON K1N 6N5, Canada. ${ }^{239}$ Division of Anatomic Pathology, Mayo Clinic, Rochester, MN 55905, USA. ${ }^{240}$ Division of Cancer Epidemiology and Genetics, National Cancer Institute, National Institutes of Health, Bethesda, MD 20892, USA. ${ }^{241}$ Illawarra Shoalhaven Local Health District L3 Illawarra Cancer Care Centre, Wollongong Hospital, Wollongong, NSW 2500, Australia. ${ }^{242}$ BioForA, French National Institute for Agriculture, Food, and Environment (INRAE), ONF, Orléans, France. ${ }^{243}$ Department of Biostatistics, Bloomberg School of Public Health, Johns Hopkins University, Baltimore, MD 21218, USA. ${ }^{244}$ University of California San Diego, San Diego, CA 92093 , USA. ${ }^{245}$ Division of Experimental Pathology, Mayo Clinic, Rochester, MN 55905, USA. ${ }^{246}$ Centre for Cancer Research, The Westmead Institute for Medical Research, University of Sydney, Sydney, NSW 2006, Australia. ${ }^{247}$ Department of Gynaecological Oncology, Westmead Hospital, Sydney, NSW 2145, Australia. ${ }^{248}$ PDXen Biosystems Inc, Seoul, South Korea. ${ }^{249}$ Korea Advanced Institute of Science and Technology, Daejeon, South Korea. ${ }^{250}$ Electronics and Telecommunications Research Institute, Daejeon, South Korea. ${ }^{251}$ Institut National du Cancer (INCA), Boulogne-

Billancourt, France. ${ }^{252}$ Division of Medical Oncology, National Cancer Centre, Singapore 169610, Singapore. ${ }^{253}$ Medical Oncology, University and Hospital Trust of Verona, Verona 37129, Italy. ${ }^{254}$ Department of Pediatrics, University Hospital Schleswig-Holstein, 23562 Kiel, Germany. ${ }^{255}$ Hepatobiliary/Pancreatic Surgical Oncology Program, University Health Network, Toronto, ON M5G 1L7, Canada. ${ }^{256}$ School of Biological Sciences, University of Auckland, Auckland 1010, New Zealand. ${ }^{257}$ Department of Surgery, University of Melbourne, Parkville, VIC 3010, Australia. ${ }^{258}$ The Murdoch Children's Research Institute, Royal Children's Hospital, Parkville, VIC 3052, Australia. ${ }^{259}$ Walter and Eliza Hall Institute, Parkville, VIC 3052 , Australia. ${ }^{260}$ Lunenfeld-Tanenbaum Research Institute, Mount Sinai Hospital, Toronto, ON M5G 1X5, Canada. ${ }^{261}$ University of East Anglia, Norwich NR4 7TJ, UK. ${ }^{262}$ Norfolk and Norwich University Hospital NHS Trust, Norwich NR4 7UY, UK. ${ }^{263}$ Victorian Institute of Forensic Medicine, Southbank, VIC 3006, Australia. ${ }^{264}$ Department of Biomedical Informatics, Harvard Medical School, Boston, MA 02115, USA. ${ }^{265}$ Department of Chemistry, Centre for Molecular Science Informatics, University of Cambridge, Cambridge CB2 1TN, UK. ${ }^{266}$ Ludwig Center at Harvard Medical School, Boston, MA 02115, USA. ${ }^{267}$ Peter MacCallum Cancer Centre, University of Melbourne, Melbourne, VIC 3010, Australia. ${ }^{268}$ Physics Division, Optimization and Systems Biology Lab, Massachusetts General Hospital, Boston, MA 02114, USA. ${ }^{269}$ Department of Medicine, Baylor College of Medicine, Houston, TX 77030, USA. ${ }^{270}$ University of Cologne, 50923 Cologne, Germany. ${ }^{271}$ International Genomics Consortium, Phoenix, AZ 85004, USA. ${ }^{272}$ Genomics Research Program, Ontario Institute for Cancer Research, Toronto, ON M5G OA3, Canada. ${ }^{273}$ Barking Havering and Redbridge University Hospitals NHS Trust, Romford, UK. ${ }^{274}$ Children's Hospital at Westmead, University of Sydney, Sydney, NSW 2006, Australia. ${ }^{275}$ Department of Medicine, Section of Endocrinology, University and Hospital Trust of Verona, 37129 Verona, Italy. ${ }^{276}$ Computational Biology Center, Memorial Sloan Kettering Cancer Center, New York, NY 10065, USA. ${ }^{277}$ Department of Biology, , ETH Zurich, Zürich, Switzerland. ${ }^{278}$ Department of Computer Science, ETH Zurich, Zurich, Switzerland. ${ }^{279}$ SIB Swiss Institute of Bioinformatics, Lausanne, Switzerland. ${ }^{280}$ Weill Cornell Medical College, New York, NY 10021 , USA. ${ }^{281}$ Academic Department of Medical Genetics, University of Cambridge, Addenbrooke's Hospital, Cambridge CB2 1TN, UK. ${ }^{282}$ MRC Cancer Unit, University of Cambridge, Cambridge CB2 1TN, UK. ${ }^{283}$ Departments of Pediatrics and Genetics, University of North Carolina at Chapel Hill, Chapel Hill, NC 27599, USA. ${ }^{284}$ Seven Bridges Genomics, Charlestown, MA 02129, USA. ${ }^{285}$ Annai Systems, Inc, Carlsbad, CA 92013, USA. ${ }^{286}$ Department of Pathology, General Hospital of Treviso, Department of Medicine, University of Padua, 35122 Treviso, Italy. ${ }^{287}$ Department of Computational Biology, University of Lausanne, 1015 Lausanne, Switzerland. ${ }^{288}$ Department of Genetic Medicine and Development, University of Geneva Medical School, 1205 CHGeneva, Switzerland. ${ }^{289}$ Swiss Institute of Bioinformatics, University of Geneva, 1205 CH Geneva, Switzerland. ${ }^{290}$ The Francis Crick Institute, London NW1 1AT, UK. ${ }^{291}$ University of Leuven, 3000 Leuven, Belgium. ${ }^{292}$ Computational and Systems Biology, Genome Institute of Singapore, Singapore 138672, Singapore. ${ }^{293}$ School of Computing, National University of Singapore, Singapore 119077, Singapore. ${ }^{294}$ Big Data Institute, Li Ka Shing Centre, University of Oxford, Oxford OX1 2JD, UK. ${ }^{295}$ The Edward S. Rogers Sr. Department of Electrical and Computer Engineering, University of Toronto, Toronto, ON M5S, Canada. ${ }^{296}$ Breast Cancer Translational Research Laboratory JC Heuson, Institut Jules Bordet, 1000 Brussels, Belgium. ${ }^{297}$ Department of Oncology, Laboratory for Translational Breast Cancer Research, KU Leuven, 3000 Leuven, Belgium. ${ }^{298}$ Institute for Research in Biomedicine (IRB Barcelona), The Barcelona Institute of Science and Technology, 08036 Barcelona, Spain. ${ }^{299}$ Research Program on Biomedical Informatics, Universitat Pompeu Fabra, 08002 Barcelona, Spain. ${ }^{300}$ Division of Medical Oncology, Princess Margaret Cancer Centre, Toronto, ON M5G 2C1, Canada. ${ }^{301}$ Department of Physiology and Biophysics, Weill Cornell Medicine, New York, NY 10065, USA. ${ }^{302}$ Institute for Computational Biomedicine, Weill Cornell Medicine, New York, NY 10065, USA. ${ }^{303}$ Department of Pathology, UPMC Shadyside, Pittsburgh, PA 15232, USA. ${ }^{304}$ Independent Consultant, Wellesley, USA. ${ }^{305}$ Department of Cell and Molecular Biology, Science for Life Laboratory, Uppsala University, 75236 Uppsala, Sweden. ${ }^{306}$ Hefei University of Technology, Anhui, China. ${ }^{307}$ Translational Cancer Research Unit, GZA Hospitals St.Augustinus, Center for Oncological Research, Faculty of Medicine and Health Sciences, University of Antwerp, Antwerp 2000, Belgium. ${ }^{308}$ University of Pennsylvania, Philadelphia, PA 19104, USA. ${ }^{309}$ The Wellcome Trust, London NW1 2BE, UK. ${ }^{310}$ Department of Pathology, Queen Elizabeth University Hospital, Glasgow G51 4TF, UK. ${ }^{311}$ Department of Genetics and Computational Biology, QIMR Berghofer Medical Research Institute, Brisbane, QLD 4006, Australia. ${ }^{312}$ Department of Oncology, Centre for Cancer Genetic Epidemiology, University of Cambridge, Cambridge CB2 1TN, UK.

${ }^{313}$ Department of Public Health and Primary Care, Centre for Cancer Genetic Epidemiology, University of Cambridge, Cambridge CB2 1TN, UK. ${ }^{314}$ Prostate Cancer Canada, Toronto, ON M5C 1M1, Canada. ${ }^{315}$ University of Cambridge, Cambridge CB2 1TN, UK. ${ }^{316}$ Department of Laboratory Medicine, Translational Cancer Research, Lund University Cancer Center at Medicon Village, Lund University, Lund, Sweden. ${ }^{317}$ CIBER Epidemiología y Salud Pública (CIBERESP), Madrid, Spain. ${ }^{318}$ Research Group on Statistics, Econometrics and Health (GRECS), UdG, Barcelona, Spain. ${ }^{319}$ Quantitative Genomics Laboratories (qGenomics), Barcelona, Spain. ${ }^{320}$ Icelandic Cancer Registry, Icelandic Cancer Society, Reykjavik, Iceland. ${ }^{321}$ State Key Laboratory of Cancer Biology, and Xijing Hospital of Digestive Diseases, Fourth Military Medical University, Shaanxi, China. ${ }^{322}$ Department of Medicine (DIMED), Surgical Pathology Unit, University of Padua, 35122 Padua, Italy. ${ }^{323}$ Department of Cancer Biology, Dana-Farber Cancer Institute, Boston, MA 02215, USA. ${ }^{324}$ Rigshospitalet, Copenhagen, Denmark. ${ }^{325}$ Center for Cancer Genomics, National Cancer Institute, National Institutes of Health, Bethesda, MD 20814, USA. ${ }^{326}$ Department of Biochemistry and Molecular Medicine, University of Montreal, Montreal, QC H3T 1J4, Canada. ${ }^{327}$ Australian Institute of Tropical Health and Medicine, James Cook University, Douglas, QLD 4811, Australia. ${ }^{328}$ Department of Neuro-Oncology, Istituto Neurologico Besta, Milano, Italy. ${ }^{329}$ Bioplatforms Australia, North Ryde, NSW, Australia. ${ }^{330}$ Department of Pathology (Research), University College London Cancer Institute, London WC1E 6DD, UK. ${ }^{331}$ Department of Surgical Oncology, Princess Margaret Cancer Centre, Toronto, ON M5G 2C1, Canada. ${ }^{332}$ Department of Medical Oncology, Josephine Nefkens Institute and Cancer Genomics Centre, Erasmus Medical Center, 3015 GD Rotterdam, The Netherlands. ${ }^{333}$ The University of Queensland Thoracic Research Centre, The Prince Charles Hospital, Brisbane, QLD 4032, Australia. ${ }^{334} \mathrm{CIBIO} /$ InBIO-Research Center in Biodiversity and Genetic Resources, Universidade do Porto, 4099-002 Vairão, Portugal. ${ }^{335} \mathrm{HCA}$ Laboratories, 
London, UK. ${ }^{336}$ University of Liverpool, Liverpool L69 3BX, UK. ${ }^{337}$ The Azrieli Faculty of Medicine, Bar-Ilan University, Safed 5290002, Israel. ${ }^{338}$ Department of Neurosurgery, University of Florida, Gainesville, FL 32611, USA. ${ }^{339}$ Department of Pathology, Graduate School of Medicine, University of Tokyo, Tokyo 113-8654, Japan. ${ }^{340}$ National Genotyping Center, Institute of Biomedical Sciences, Academia Sinica, Taipei, Taiwan. ${ }^{341}$ University of Milano Bicocca, Monza 20126, Italy. ${ }^{342}$ Department of Pathology, Oslo University Hospital Ulleval, 0450 Oslo, Norway. ${ }^{343}$ Center for Biomedical Informatics, Harvard Medical School, Boston, MA 02115, USA. ${ }^{344}$ Office of Cancer Genomics, National Cancer Institute, National Institutes of Health, Bethesda, MD 20892, USA. ${ }^{345}$ Cancer Epigenomics, German Cancer Research Center (DKFZ), 69120 Heidelberg, Germany. ${ }^{346}$ Department of Cancer Biology, The University of Texas MD Anderson Cancer Center, Houston, TX 77030, USA. ${ }^{347}$ Department of Surgical Oncology, The University of Texas MD Anderson Cancer Center, Houston, TX 77030, USA. ${ }^{348}$ Department of Pathology, Memorial Sloan Kettering Cancer Center, New York, NY 10065, USA. ${ }^{349}$ Division of Gastroenterology and Hepatology, Mayo Clinic, Rochester, MN 55905, USA. ${ }^{350}$ University of Sydney, Sydney, NSW 2006, Australia. ${ }^{351}$ University of Oxford, Oxford OX1 2JD, UK. ${ }^{352}$ Cambridge University Hospitals NHS Foundation Trust, Cambridge CB2 OQQ, UK. ${ }^{353}$ Department of Surgery, Academic Urology Group, University of Cambridge, Cambridge CB2 OQQ, UK. ${ }^{354}$ Department of Medicine II, University of Würzburg, 97070 Wuerzburg, Germany. ${ }^{355}$ Sylvester Comprehensive Cancer Center, University of Miami, Miami, FL 33146, USA. ${ }^{356}$ Institut Hospital del Mar d'Investigacions Mèdiques (IMIM), Barcelona, Spain. ${ }^{357}$ Genome Integrity and Structural Biology Laboratory, National Institute of Environmental Health Sciences (NIEHS), Durham, NC 27709, USA. ${ }^{358}$ St. Thomas's Hospital, London SE1 7EH, UK. ${ }^{359}$ Osaka International Cancer Center, Osaka, Japan. ${ }^{360}$ Department of Pathology, Skåne University Hospital, Lund University, Lund, Sweden. ${ }^{361}$ Department of Medical Oncology, Beatson West of Scotland Cancer Centre, Glasgow G12 OYN, UK. ${ }^{362}$ Centre for Cancer Research, Victorian Comprehensive Cancer Centre, University of Melbourne, Melbourne, VIC 3010, Australia. ${ }^{363}$ Department of Medicine, Section of Hematology/Oncology, University of Chicago, Chicago, IL 60637, USA. ${ }^{364}$ German Center for Infection Research (DZIF), Partner Site Hamburg-Borstel-Lübeck-Riems,

Hamburg, Germany. ${ }^{365}$ Department of Biotechnology, Ministry of Science and Technology, Government of India, New Delhi, Delhi 110016, India. ${ }^{366}$ National Cancer Centre Singapore, Singapore 169610, Singapore. ${ }^{367}$ Brandeis University, Waltham, MA 02453, USA. ${ }^{368}$ Department of Internal Medicine, Stanford University, Stanford, CA 94305, USA. ${ }^{369}$ The University of Texas Health Science Center at Houston, Houston, TX 77030, USA. ${ }^{370}$ Imperial College NHS Trust, Imperial College, London, INY SW7 2BU, UK. ${ }^{371}$ Senckenberg Institute of Pathology, University of Frankfurt Medical School, 60323 Frankfurt, Germany. ${ }^{372}$ Department of Medicine, Division of Biomedical Informatics, UC San Diego School of Medicine, San Diego, CA 92093, USA. ${ }^{373}$ Center for Precision Health, School of Biomedical Informatics, The University of Texas Health Science Center, Houston, TX 77030, USA. ${ }^{374}$ Oxford Nanopore Technologies, New York, NY OX4 4DQ, USA. ${ }^{375}$ Institute of Medical Science, University of Tokyo, Tokyo 113-8654, Japan. ${ }^{376}$ Wakayama Medical University, Wakayama 641-8510, Japan. ${ }^{377}$ Department of Internal Medicine, Division of Medical Oncology, Lineberger Comprehensive Cancer Center, University of North Carolina at Chapel Hill, Chapel Hill, NC 27599, USA. ${ }^{378}$ University of Tennessee Health Science Center for Cancer Research, Memphis, TN 38163, USA. ${ }^{379}$ Department of Histopathology, Salford Royal NHS Foundation Trust, Salford M6 8 HD, UK. ${ }^{380}$ Faculty of Biology, Medicine and Health, University of Manchester, Manchester M13 9PL, UK. ${ }^{381}$ Peking University, 100871 Beijing, China. ${ }^{382}$ Children's Hospital of Philadelphia, Philadelphia, PA 19104, USA. ${ }^{383}$ Karolinska Institute, 17177 Stockholm, Sweden. ${ }^{384}$ The Donnelly Centre, University of Toronto, Toronto, ON M5S, Canada. ${ }^{385}$ Department of Medical Genetics, College of Medicine, Hallym University, Chuncheon, South Korea. ${ }^{386}$ Department of Experimental and Health Sciences, Institute of Evolutionary Biology (UPF-CSIC), Universitat Pompeu Fabra, 08002 Barcelona, Spain. ${ }^{387}$ Health Data Science Unit, University Clinics, Heidelberg, Germany. ${ }^{388}$ Hokkaido University, Sapporo 060-0808, Japan. ${ }^{389}$ Department of Pathology and Clinical Laboratory, National Cancer Center Hospital, Tokyo 104-0045, Japan. ${ }^{390}$ Department of Genetics, University of North Carolina at Chapel Hill, Chapel Hill, NC 27599, USA. ${ }^{391}$ Computational Biology, Leibniz Institute on Aging-Fritz Lipmann Institute (FLI), 07745 Jena, Germany. ${ }^{392}$ University of Melbourne Centre for Cancer Research, Melbourne, VIC 3010, Australia. ${ }^{393}$ University of Nebraska Medical Center, Omaha, NE 68198, USA. ${ }^{394}$ Syntekabio Inc, Daejeon, South Korea. ${ }^{395}$ Department of Pathology, Academic Medical Center, Amsterdam, 1105 AZ, The Netherlands. ${ }^{396}$ China National GeneBank-Shenzhen, Shenzhen, China. ${ }^{397}$ Division of Molecular Genetics, German Cancer Research Center (DKFZ), 69120 Heidelberg, Germany. ${ }^{398}$ Icahn School of Medicine at Mount Sinai, New York, NY 10029, USA. ${ }^{399}$ AbbVie, North Chicago, IL 60064, USA. ${ }^{400}$ Institute of Pathology, Charité - University Medicine Berlin, Berlin, Germany. ${ }^{401}$ Centre for Translational and Applied Genomics, British Columbia Cancer Agency, Vancouver, BC V8R 6V5, Canada. ${ }^{402}$ Edinburgh Royal Infirmary, Edinburgh EH16 4SA, UK. ${ }^{403}$ Berlin Institute for Medical Systems Biology, Max Delbrück Center for Molecular Medicine, 13125 Berlin, Germany. ${ }^{404}$ Department of Pediatric Immunology, Hematology and Oncology, University Hospital, Heidelberg, 69120 Heidelberg, Germany. ${ }^{405}$ German Cancer Research Center (DKFZ), 69120 Heidelberg, Germany. ${ }^{406}$ Heidelberg Institute for Stem Cell Technology and Experimental Medicine (HI-STEM), 69120 Heidelberg, Germany. ${ }^{407}$ Institute for Computational Biomedicine, Weill Cornell Medical College, New York, NY 10021, USA. ${ }^{408}$ New York Genome Center, New York, NY 10013, USA. ${ }^{409}$ Department of Urology, James Buchanan Brady Urological Institute, Johns Hopkins University School of Medicine, Baltimore, MD 21205, USA. ${ }^{410}$ Department of Preventive Medicine, Graduate School of Medicine, The University of Tokyo, Tokyo 113-8654, Japan. ${ }^{411}$ Department of Molecular and Cellular Biology, Baylor College of Medicine, Houston, TX 77030, USA. ${ }^{412}$ Department of Pathology and Immunology, Baylor College of Medicine, Houston, TX 77030, USA. ${ }^{413}$ Michael E. DeBakey Veterans Affairs Medical Center, Houston, TX 77030, USA. ${ }^{414}$ Technical University of Denmark, 2800 Lyngby, Denmark. ${ }^{415}$ Department of Pathology, College of Medicine, Hanyang University, Seoul, South Korea. ${ }^{416}$ Academic Unit of Surgery, School of Medicine, College of Medical, Veterinary and Life Sciences, University of Glasgow, Glasgow Royal Infirmary, Glasgow G12 8QQ, UK. ${ }^{417}$ Department of Pathology, Asan Medical Center, College of Medicine, Ulsan University, Songpa-gu, Seoul, South Korea. ${ }^{418}$ Science Writer, Garrett Park, MD, USA. ${ }^{419}$ International Cancer Genome Consortium (ICGC)/ICGC Accelerating Research in Genomic Oncology (ARGO) Secretariat, Ontario Institute for Cancer Research, Toronto, ON M5G OA3, Canada. ${ }^{420}$ University of Ljubljana, 1000 Ljubljana, Slovenia. ${ }^{421}$ Department of Public Health Sciences, University of Chicago, Chicago, IL 60637, USA. ${ }^{422}$ Research Institute, NorthShore University HealthSystem, Evanston, IL 60201, USA. ${ }^{423}$ Department for Biomedical Research, University of Bern, Bern 3012, Switzerland. ${ }^{424}$ Centre of Genomics and Policy, McGill University and Génome Québec Innovation Centre, Montreal, QC H3A 0G4, Canada. ${ }^{425}$ Carolina Center for Genome Sciences, University of North Carolina at Chapel Hill, Chapel Hill, NC 27599, USA. ${ }^{426}$ Hopp Children's Cancer Center (KiTZ), Heidelberg, Germany. ${ }^{427}$ Pediatric Glioma Research Group, German Cancer Research Center (DKFZ), 69120 Heidelberg, Germany. ${ }^{428}$ Cancer Research UK, London NG34 7SY, UK. ${ }^{429}$ Indivumed GmbH, Hamburg, Germany. ${ }^{430}$ University Hospital Zurich, 8091 Zurich, Switzerland. ${ }^{431}$ Clinical Bioinformatics, Swiss Institute of Bioinformatics, 1015 Geneva, Switzerland. ${ }^{432}$ Institute for Pathology and Molecular Pathology, University Hospital Zurich, 8091 Zurich, Switzerland. ${ }^{433}$ Institute of Molecular Life Sciences, University of Zurich, 8091 Zurich, Switzerland. ${ }^{434}$ MRC Human Genetics Unit, MRC IGMM, University of Edinburgh, Edinburgh EH8 9YL, UK. ${ }^{435}$ Women's Cancer Program at the Samuel Oschin Comprehensive Cancer Institute, Cedars-Sinai Medical Center, Los Angeles, CA 90048, USA. ${ }^{436}$ Department of Biology, Bioinformatics Group, Division of Molecular Biology, Faculty of Science, University of Zagreb, 10000 Zagreb, Croatia. ${ }^{437}$ Department for Internal Medicine II, University Hospital Schleswig-Holstein, 23562 Kiel, Germany. ${ }^{438}$ Genetics and Molecular Pathology, SA Pathology, Adelaide, SA 5011, Australia. ${ }^{439}$ Department of Gastric Surgery, National Cancer Center Hospital, Tokyo, Japan. ${ }^{440}$ Department of Bioinformatics, Division of Cancer Genomics, National Cancer Center Research Institute, Tokyo, Japan. ${ }^{441}$ A.A. Kharkevich Institute of Information Transmission Problems, Moscow, Russia. ${ }^{442}$ Oncology and Immunology, Dmitry Rogachev National Research Center of Pediatric Hematology, Moscow, Russia. ${ }^{443}$ Skolkovo Institute of Science and Technology, Moscow, Russia. ${ }^{444}$ Department of Surgery, The George Washington 
University, School of Medicine and Health Science, Washington, DC 20052, USA. ${ }^{445}$ Endocrine Oncology Branch, Center for Cancer Research, National Cancer Institute, National Institutes of Health, Bethesda, MD 20892, USA. ${ }^{446}$ Melanoma Institute Australia, Macquarie University, Sydney, NSW 2109, Australia. ${ }^{447}$ MIT Computer Science and Artificial Intelligence Laboratory, Massachusetts Institute of Technology, Cambridge, MA 02139, USA. ${ }^{448}$ Tissue Pathology and Diagnostic Oncology, Royal Prince Alfred Hospital, Sydney, NSW 2050, Australia. ${ }^{449}$ Cholangiocarcinoma Screening and Care Program and Liver Fluke and Cholangiocarcinoma Research Centre, Faculty of Medicine, Khon Kaen University, Khon Kaen 40002, Thailand. ${ }^{450}$ Controlled Department and Institution, New York, NY, USA. ${ }^{451}$ Englander Institute for Precision Medicine, Weill Cornell Medicine, New York, NY 10065, USA. ${ }^{452}$ National Cancer Center, Gyeonggi, South Korea. ${ }^{453}$ Health Sciences Department of Biomedical Informatics, University of California San Diego, La Jolla, CA 92093, USA. ${ }^{454}$ Research Core Center, National Cancer Centre Korea, Goyang-si, South Korea. ${ }^{455}$ Department of Health Sciences and Technology, Sungkyunkwan University School of Medicine, Seoul, South Korea. ${ }^{456}$ Samsung Genome Institute, Seoul, South Korea. ${ }^{457}$ Breast Oncology Program, Dana-Farber/Brigham and Women's Cancer Center, Boston, MA 02215, USA. ${ }^{458}$ Department of Surgery, Memorial Sloan Kettering Cancer Center, New York, NY 10065, USA. ${ }^{459}$ Division of Breast Surgery, Brigham and Women's Hospital, Boston, MA 02115, USA. ${ }^{460}$ Integrative Bioinformatics Support Group, National Institute of Environmental Health Sciences (NIEHS), Durham, NC 27709, USA. ${ }^{461}$ Department of Clinical Science, University of Bergen, 5007 Bergen, Norway. ${ }^{462}$ Center For Medical Innovation, Seoul National University Hospital, Seoul, South Korea. ${ }^{463}$ Department of Internal Medicine, Seoul National University Hospital, Seoul, South Korea. ${ }^{464}$ Institute of Computer Science, Polish Academy of Sciences, Warsawa, Poland. ${ }^{465}$ Functional and Structural Genomics, German Cancer Research Center (DKFZ), 69120 Heidelberg, Germany. ${ }^{466}$ Laboratory of Translational Genomics, Division of Cancer Epidemiology and Genetics, National Cancer Institute, National Institutes of Health, Bethesda, MD 20892, USA. ${ }^{467}$ Institute for Medical Informatics Statistics and Epidemiology, University of Leipzig, 04109 Leipzig, Germany. ${ }^{468}$ Morgan Welch Inflammatory Breast Cancer Research Program and Clinic, The University of Texas MD Anderson Cancer Center, Houston, TX 77030, USA. ${ }^{469}$ Department of Hematology and Oncology, Georg-Augusts-University of Göttingen, 37073 Göttingen, Germany. ${ }^{470}$ Institute of Cell Biology (Cancer Research), University of Duisburg-Essen, 47057 Essen, Germany. ${ }^{471}$ King's College London and Guy's and St. Thomas' NHS Foundation Trust, London WC2R 2LS, UK. ${ }^{472}$ Center for Epigenetics, Van Andel Research Institute, Grand Rapids, MI 49503, USA. ${ }^{473}$ The University of Queensland Centre for Clinical Research, Royal Brisbane and Women's Hospital, Herston, QLD 4029, Australia. ${ }^{474}$ Department of Pediatric Oncology and Hematology, University of Cologne, 50923 Cologne, Germany. ${ }^{475}$ University of Düsseldorf, 40225 Düsseldorf, Germany. ${ }^{476}$ Department of Pathology, Institut Jules Bordet, Brussels 1000, Belgium. ${ }^{477}$ Institute of Biomedicine, Sahlgrenska Academy at University of Gothenburg, 41390 Gothenburg, Sweden. ${ }^{478}$ Children's Medical Research Institute, Sydney, NSW 2145, Australia. ${ }^{479}$ ILSbio, LLC Biobank, Chestertown, MD, USA. ${ }^{480}$ Division of Genetics and Genomics, Boston Children's Hospital, Harvard Medical School, Boston, MA 02115, USA ${ }^{481}$ Institute for Bioengineering and Biopharmaceutical Research (IBBR), Hanyang University, Seoul, South Korea. ${ }^{482}$ Department of Statistics, University of California Santa Cruz, Santa Cruz, CA 95064, USA. ${ }^{483}$ Department of Vertebrate Genomics/Otto Warburg Laboratory Gene Regulation and Systems Biology of Cancer, Max Planck Institute for Molecular Genetics, 14195 Berlin, Germany. ${ }^{484}$ McGill University and Genome Quebec Innovation Centre, Montreal, QC H3A OG4, Canada. ${ }^{485}$ Gynecologic Oncology, NYU Laura and Isaac Perlmutter Cancer Center, New York University, New York, NY 10003, USA. ${ }^{486}$ Division of Oncology, Stem Cell Biology Section, Washington University School of Medicine, St. Louis, MO 63110, USA. ${ }^{487}$ Harvard University, Cambridge, MA 02138, USA. ${ }^{488}$ Urologic Oncology Branch, Center for Cancer Research, National Cancer Institute, National Institutes of Health, Bethesda, MD 20892, USA. ${ }^{489}$ University of Oslo, 0315 Oslo, Norway. ${ }^{490}$ University of Toronto, Toronto, ON M5S, Canada. ${ }^{491}$ School of Life Sciences, Peking University, Beijing, China. ${ }^{492}$ Leidos Biomedical Research, Inc, McLean, VA, USA. ${ }^{493}$ Hematology, Hospital Clinic, Institut d'Investigacions Biomèdiques August Pi i Sunyer (IDIBAPS), University of Barcelona, 08007 Barcelona, Spain. ${ }^{494}$ Second Military Medical University, Shanghai, China. ${ }^{495}$ Chinese Cancer Genome Consortium, Shenzhen, China. ${ }^{496}$ Department of Medical Oncology, Beijing Hospital, Beijing, China. ${ }^{497}$ Laboratory of Molecular Oncology, Key Laboratory of Carcinogenesis and Translational Research (Ministry of Education), Peking University Cancer Hospital and Institute, Beijing, China. ${ }^{498}$ School of Medicine/School of Mathematics and Statistics, University of St. Andrews, St. Andrews, Fife KY16 9AJ, UK. ${ }^{499}$ Department of Biochemistry and Molecular Biology, Faculty of Medicine, University Institute of Oncology-IUOPA, Oviedo, Spain. ${ }^{500}$ Institut Bergonié, Bordeaux, France. ${ }^{501}$ Cancer Unit, MRC University of Cambridge, Cambridge CB2 1TN, UK. ${ }^{502}$ Department of Pathology and Laboratory Medicine, Center for Personalized Medicine, Children's Hospital Los Angeles, Los Angeles, CA 90027, USA. ${ }^{503}$ John Curtin School of Medical Research, Canberra, ACT 2601, Australia. ${ }^{504}$ MVZ Department of Oncology, PraxisClinic am Johannisplatz, Leipzig, Germany. ${ }^{505}$ Department of Information Technology, Ghent University, Ghent, Belgium. ${ }^{506}$ Department of Plant Biotechnology and Bioinformatics, Ghent University, Ghent, Belgium. ${ }^{507}$ Institute for Genomic Medicine, Nationwide Children's Hospital, Columbus, OH 43205, USA. ${ }^{508}$ Department of Surgery, Duke University, Durham, NC 27708, USA. ${ }^{509}$ Institut Català de Paleontologia Miquel Crusafont, Universitat Autònoma de Barcelona, Barcelona, Spain. ${ }^{510}$ University of Glasgow, Glasgow G12 8QQ, UK. ${ }^{511}$ Institut d'Investigacions Biomèdiques August Pi i Sunyer (IDIBAPS),

Barcelona, Spain. ${ }^{512}$ Department of Surgery and Cancer, Imperial College, London, INY SW7 2BU, UK. ${ }^{513}$ Applications Department, Oxford Nanopore Technologies, Oxford OX4 4DQ, UK. ${ }^{514}$ Department of Obstetrics, Gynecology and Reproductive Services, University of California San Francisco, San Francisco, CA 94110, USA. ${ }^{515}$ Department of Biochemistry and Molecular Medicine, University California at Davis, Sacramento, CA 95616, USA. ${ }^{516}$ STTARR Innovation Facility, Princess Margaret Cancer Centre, Toronto, ON M5G 2C1, Canada. ${ }^{517}$ Discipline of Surgery, Western Sydney University, Penrith, NSW 2150, Australia. ${ }^{518}$ Department of Genetics, Lineberger Comprehensive Cancer Center, University of North Carolina at Chapel Hill, Chapel Hill, NC 27599, USA. ${ }^{519}$ Departments of Neurology and Neurosurgery, Henry Ford Hospital, Detroit, MI 48202, USA. ${ }^{520}$ Institute of Pathology, University Medical Center Hamburg-Eppendorf, 20251 Hamburg, Germany. ${ }^{521}$ Department of Health Sciences, Faculty of Medical Sciences, Kyushu University, Fukuoka, Japan. ${ }^{522}$ Heidelberg Academy of Sciences and Humanities, Heidelberg, Germany. ${ }^{523}$ Department of Clinical Pathology, University of Melbourne, Melbourne, VIC 3010, Australia. ${ }^{524}$ Department of Pathology, Roswell Park Cancer Institute, Buffalo, NY 14203 , USA. ${ }^{525}$ Department of Computer Science, University of Helsinki, 00100 Helsinki, Finland. ${ }^{526}$ Institute of Biotechnology, University of Helsinki, 00100 Helsinki, Finland. ${ }^{527}$ Organismal and Evolutionary Biology Research Programme, University of Helsinki, 00100 Helsinki, Finland. ${ }^{528}$ Department of Obstetrics and Gynecology, Division of Gynecologic Oncology, Washington University School of Medicine, St. Louis, MO 63110, USA. 529Penrose St. Francis Health Services, Colorado Springs, CO 80923, USA. ${ }^{530}$ Institute of Pathology, Ulm University and University Hospital of Ulm, Ulm 89081 , Germany. ${ }^{531}$ National Cancer Center, Tokyo, Japan. ${ }^{532}$ Genome Institute of Singapore, Singapore 138672, Singapore. ${ }^{533}$ German Cancer Aid, Bonn, Germany. ${ }^{534}$ Programme in Cancer and Stem Cell Biology, Centre for Computational Biology, Duke-NUS Medical School, 169857 Singapore, Singapore. ${ }^{535}$ The Chinese University of Hong Kong, Shatin, NT, Hong Kong, China. ${ }^{536}$ Fourth Military Medical University, Shaanxi, China. ${ }^{537}$ The University of Cambridge School of Clinical Medicine, Cambridge CB2 1TN, UK. ${ }^{538}$ St. Jude Children's Research Hospital, Memphis, TN 38105 , USA. ${ }^{539}$ University Health Network, Princess Margaret Cancer Centre, Toronto, ON M5G 1L7, Canada. ${ }^{540}$ Center for Biomolecular Science and Engineering, University of California Santa Cruz, Santa Cruz, CA 95064, USA. ${ }^{541}$ Department of Medicine, University of Chicago, Chicago, IL 60637, USA. ${ }^{542}$ Department of Neurology, Mayo Clinic, Rochester, MN 55905, USA. ${ }^{543}$ Cambridge Oesophagogastric Centre, Cambridge University Hospitals NHS Foundation Trust, Cambridge CB2 0QQ, UK. ${ }^{544}$ Department of Computer Science, Carleton College, Northfield, MN 55057, USA. ${ }^{545}$ Institute of Cancer Sciences, College of Medical Veterinary and Life Sciences, University of Glasgow, Glasgow G12 8QQ, UK. ${ }^{546}$ Department of Epidemiology, University of Alabama at Birmingham, Birmingham, AL 35294, USA. ${ }^{547}$ HudsonAlpha Institute for Biotechnology, Huntsville, AL 
35806, USA. ${ }^{548} \mathrm{O}^{\prime} \mathrm{Neal}$ Comprehensive Cancer Center, University of Alabama at Birmingham, Birmingham, AL 35294, USA. ${ }^{549}$ Department of Pathology, Keio University School of Medicine, Tokyo, Japan. ${ }^{550}$ Department of Hepatobiliary and Pancreatic Oncology, National Cancer Center Hospital, Tokyo, Japan. ${ }^{551}$ Sage Bionetworks, Seattle, WA, USA. ${ }^{552}$ Lymphoma Genomic Translational Research Laboratory, National Cancer Centre, Singapore 98121, Singapore. ${ }^{553}$ Department of Clinical Pathology, Robert-Bosch-Hospital, 70376 Stuttgart, Germany. ${ }^{554}$ Department of Cell and Systems Biology, University of Toronto, Toronto, ON M5S, Canada. ${ }^{555}$ Department of Biosciences and Nutrition, Karolinska Institutet, 17177 Stockholm, Sweden. ${ }^{556}$ Center for Liver Cancer, Research Institute and Hospital, National Cancer Center, Gyeonggi, South Korea. ${ }^{557}$ Division of Hematology-Oncology, Samsung Medical Center, Sungkyunkwan University School of Medicine, Seoul, South Korea. ${ }^{558}$ Samsung Advanced Institute for Health Sciences and Technology, Sungkyunkwan University School of Medicine, Seoul, South Korea. ${ }^{559}$ Cheonan Industry-Academic Collaboration Foundation, Sangmyung University, Cheonan, South Korea. ${ }^{560}$ NYU Langone Medical Center, New York, NY 10016, USA. ${ }^{561}$ Department of Hematology and Medical Oncology, Cleveland Clinic, Cleveland, OH 44195, USA. 562Department of Health Sciences Research, Mayo Clinic, Rochester, MN 55905, USA. ${ }^{563}$ Helen F. Graham Cancer Center at Christiana Care Health Systems, Newark, DE 19713, USA. ${ }^{564}$ Heidelberg University Hospital, 69120 Heidelberg, Germany. ${ }^{565}$ CSRA Incorporated, Fairfax, VA, USA. ${ }^{566}$ Research Department of Pathology, University College London Cancer Institute, London WC1E 6DD, UK. ${ }^{567}$ Department of Research Oncology, Guy's Hospital, King's Health Partners AHSC, King's College London School of Medicine, London WC2R 2LS, UK. ${ }^{568}$ Faculty of Medicine and Health Sciences, Macquarie University, Sydney, NSW 2109 , Australia. ${ }^{569}$ University Hospital of Minjoz, INSERM UMR 1098, Besançon, France. ${ }^{570}$ Spanish National Cancer Research Centre, Madrid 28029, Spain. ${ }^{571}$ Center of Digestive Diseases and Liver Transplantation, Fundeni Clinical Institute, Bucharest, Romania. ${ }^{572}$ Cureline, Inc, South San Francisco, CA, USA. ${ }^{573}$ St. Luke's Cancer Centre, Royal Surrey County Hospital NHS Foundation Trust, Guildford, UK. ${ }^{574}$ Cambridge Breast Unit, Addenbrooke's Hospital, Cambridge University Hospital NHS Foundation Trust and NIHR Cambridge Biomedical Research Centre, Cambridge CB2 0QQ, UK. ${ }^{575}$ East of Scotland Breast Service, Ninewells Hospital, Aberdeen, UK. ${ }^{576}$ Department of Genetics, Microbiology and Statistics, University of Barcelona, IRSJD, IBUB, Barcelona, Spain. ${ }^{577}$ Department of Obstetrics and Gynecology, Medical College of Wisconsin, Milwaukee, WI 53226, USA. ${ }^{578} \mathrm{Hematology}$ and Medical Oncology, Winship Cancer Institute of Emory University, Atlanta, GA 30322, USA. ${ }^{579}$ Department of Computer Science, Princeton University, Princeton, NJ 08544, USA. ${ }^{580}$ Vanderbilt Ingram Cancer Center, Vanderbilt University, Nashville, TN 37235, USA. ${ }^{581}$ Ohio State University College of Medicine and Arthur G. James Comprehensive Cancer Center, Columbus, OH 43210, USA. ${ }^{582}$ Department of Surgery, Yokohama City University Graduate School of Medicine, Kanagawa 236-0027, Japan. ${ }^{583}$ Research Computing Center, University of North Carolina at Chapel Hill, Chapel Hill, NC 27599, USA. ${ }^{584}$ School of Molecular Biosciences and Center for Reproductive Biology, Washington State University, Pullman, WA 99163, USA. ${ }^{585}$ Department of Laboratory Medicine and Pathobiology, University of Toronto, Toronto, ON M5S, Canada. ${ }^{586}$ Department of Pathology, Human Oncology and Pathogenesis Program, Memorial Sloan Kettering Cancer Center, New York, NY 10065, USA. ${ }^{587}$ University Hospital Giessen, Pediatric Hematology and Oncology, Giessen, Germany. ${ }^{588}$ Oncologie Sénologie, ICM Institut Régional du Cancer, Montpellier, France. ${ }^{589}$ Institute of Clinical Molecular Biology, Christian-Albrechts-University, Kiel, Germany. ${ }^{590}$ Institute of Pathology, University of Wuerzburg, 97070 Wuerzburg, Germany. ${ }^{591}$ Department of Urology, North Bristol NHS Trust, Bristol, UK. ${ }^{592}$ SingHealth, Duke-NUS Institute of Precision Medicine, National Heart Centre Singapore, Singapore 169609, Singapore. ${ }^{593}$ Bern Center for Precision Medicine, University Hospital of Bern, University of Bern, Bern 3012, Switzerland. ${ }^{594}$ Englander Institute for Precision Medicine, Weill Cornell Medicine and New York Presbyterian Hospital, New York, NY 10065, USA. ${ }^{595}$ Meyer Cancer Center, Weill Cornell Medicine, New York, NY 10065, USA. ${ }^{596}$ Pathology and Laboratory, Weill Cornell Medical College, New York, NY 10065, USA. ${ }^{597}$ Vall d'Hebron Institute of Oncology: VHIO, Barcelona, Spain. ${ }^{598}$ General and Hepatobiliary-Biliary Surgery, Pancreas Institute, University and Hospital Trust of Verona, Verona, Italy. ${ }^{599}$ National Centre for Biological Sciences, Tata Institute of Fundamental Research, Bangalore 560065, India. ${ }^{600}$ Department of Pathology, GZA-ZNA Hospitals, Antwerp, Belgium. ${ }^{601}$ Analytical Biological Services, Inc, Wilmington, DE 19801, USA. ${ }^{602}$ Sydney Medical School, University of Sydney, Sydney, NSW 2050, Australia. ${ }^{603}$ CBio Center, Dana-Farber Cancer Institute, Harvard Medical School, Boston, MA 02115, USA. ${ }^{604}$ Department of Cell Biology, Harvard Medical School, Boston, MA 02115, USA. ${ }^{605}$ Advanced Centre for Treatment Research and Education in Cancer, Tata Memorial Centre, Navi Mumbai, Maharashtra 400012, India. ${ }^{606}$ School of Environmental and Life Sciences, Faculty of Science, The University of Newcastle, Ourimbah, NSW 2308, Australia. ${ }^{607}$ Department of Dermatology, University Hospital of Essen, 45147 Essen, Germany. ${ }^{608}$ Martini-Clinic, Prostate Cancer Center, University Medical Center Hamburg-Eppendorf, 20251 Hamburg, Germany. ${ }^{609}$ Department of General Internal Medicine, University of Kiel, 24118 Kiel, Germany. ${ }^{610}$ German Cancer Consortium (DKTK), Partner site Berlin, Berlin, Germany. ${ }^{611}$ Cancer Research Institute, Beth Israel Deaconess Medical Center, Boston, MA 02215, USA.

${ }^{612}$ University of Pittsburgh, Pittsburgh, PA 15260, USA. ${ }^{613}$ Department of Ophthalmology and Ocular Genomics Institute, Massachusetts Eye and Ear, Harvard Medical School, Boston, MA 02115, USA. ${ }^{614}$ Center for Psychiatric Genetics, NorthShore University HealthSystem, Evanston, IL 60031, USA. ${ }^{615}$ Van Andel Research Institute, Grand Rapids, MI 49503, USA. ${ }^{616}$ Laboratory of Molecular Medicine, Human Genome Center, Institute of Medical Science, University of Tokyo, Tokyo, Japan. ${ }^{617}$ Japan Agency for Medical Research and Development, Tokyo, Japan. ${ }^{618}$ Korea University, Seoul, South Korea. ${ }^{619}$ Murtha Cancer Center, Walter Reed National Military Medical Center, Bethesda, MD 20814, USA. ${ }^{620}$ Human Genetics, University of Kiel, 24118 Kiel, Germany. ${ }^{621}$ Department of Oncologic Pathology, Dana-Farber Cancer Institute, Harvard Medical School, Boston, MA 02115, USA. ${ }^{622}$ Oregon Health and Science University, Portland, OR 97239, USA. ${ }^{623}$ Center for RNA Interference and Noncoding RNA, The University of Texas MD Anderson Cancer Center, Houston, TX 77030, USA. ${ }^{624}$ Department of Experimental Therapeutics, The University of Texas MD Anderson Cancer Center, Houston, TX 77030, USA. ${ }^{625}$ Department of Gynecologic Oncology and Reproductive Medicine, The University of Texas MD Anderson Cancer Center, Houston, TX 77030, USA. ${ }^{626}$ University Hospitals Coventry and Warwickshire NHS Trust, Coventry, UK. ${ }^{627}$ Department of Radiation Oncology, Radboud University Nijmegen Medical Centre, Nijmegen 6525 GA, The Netherlands. ${ }^{628}$ Institute for Genomics and Systems Biology, University of Chicago, Chicago, IL, USA. ${ }^{629}$ Clinic for Hematology and Oncology, St.-Antonius-Hospital, 60637 Eschweiler, Germany. ${ }^{630}$ Computational and Systems Biology Program, Memorial Sloan Kettering Cancer Center, New York, NY 10065, USA. ${ }^{331}$ University of Iceland, Reykjavik, Iceland. ${ }^{632}$ Division of Computational Genomics and Systems Genetics, German Cancer Research Center (DKFZ), Heidelberg, Germany. ${ }^{633}$ Dundee Cancer Centre, Ninewells Hospital, Dundee, UK. ${ }^{634}$ Department for Internal Medicine III, University of Ulm and University Hospital of Ulm, Ulm, Germany. ${ }^{635}$ Institut Curie, INSERM Unit 830, Paris, France. ${ }^{636}$ Department of Gastroenterology and Hepatology, Yokohama City University Graduate School of Medicine, Kanagawa, Japan. ${ }^{637}$ Department of Laboratory Medicine, Radboud University Nijmegen Medical Centre, Nijmegen 6525 GA, The Netherlands. ${ }^{638}$ Division of Cancer Genome Research, German Cancer Research Center (DKFZ), Heidelberg, Germany. ${ }^{639}$ Department of General Surgery, Singapore General Hospital, Singapore, Singapore. ${ }^{640}$ Cancer Science Institute of Singapore, National University of Singapore, Singapore, Singapore. ${ }^{641}$ Department of Medical and Clinical Genetics, Genome-Scale Biology Research Program, University of Helsinki, Helsinki, Finland. ${ }^{642}$ East Anglian Medical Genetics Service, Cambridge University Hospitals NHS Foundation Trust, Cambridge, UK. ${ }^{643}$ Irving Institute for Cancer Dynamics, Columbia University, New York, NY 10027, USA. ${ }^{644}$ Institute of Molecular and Cell Biology, Singapore, Singapore. ${ }^{645}$ Laboratory of Cancer Epigenome, Division of Medical Science, National Cancer Centre Singapore, Singapore, Singapore. ${ }^{646}$ Universite Lyon, INCaSynergie, Centre Léon Bérard, Lyon, France. ${ }^{647}$ Department of Urology, Mayo Clinic, Rochester, MN 55905, USA. ${ }^{648}$ Royal National Orthopaedic Hospital-Stanmore, Stanmore, Middlesex, UK. ${ }^{649}$ Giovanni Paolo II / I.R.C.C.S. Cancer Institute, Bari, BA, Italy. ${ }^{650}$ Neuroblastoma Genomics, German Cancer Research Center (DKFZ), Heidelberg, Germany. ${ }^{651}$ Fondazione Policlinico Universitario Gemelli IRCCS, Rome, Italy, Rome, Italy. ${ }^{652}$ University 
of Verona, Verona, Italy. ${ }^{653}$ Centre National de Génotypage, CEA-Institute de Génomique, Evry, France. ${ }^{654}$ CAPHRI Research School, Maastricht University, Maastricht, ER, The Netherlands. ${ }^{655}$ Department of Biopathology, Centre Léon Bérard, Lyon, France. ${ }^{656}$ Université Claude Bernard Lyon 1 , Villeurbanne, France. ${ }^{657}$ Core Research for Evolutional Science and Technology (CREST), JST, Tokyo, Japan. ${ }^{658}$ Department of Biological Sciences, Laboratory for Medical Science Mathematics, Graduate School of Science, University of Tokyo, Yokohama, Japan. ${ }^{659}$ Department of Medical Science Mathematics, Medical Research Institute, Tokyo Medical and Dental University (TMDU), Tokyo, Japan. ${ }^{660}$ University Hospitals Birmingham NHS Foundation Trust, Birmingham, UK. ${ }^{661}$ Centre for Cancer Research and Cell Biology, Queen's University, Belfast, UK. ${ }^{662}$ Breast Medical Oncology, The University of Texas MD Anderson Cancer Center, Houston, TX 77030, USA. ${ }^{663}$ Department of Surgery, Johns Hopkins University School of Medicine, Baltimore, MD 21205, USA. ${ }^{664}$ Department of Oncology-Pathology, Science for Life Laboratory, Karolinska Institute, 17177 Stockholm, Sweden. ${ }^{665}$ School of Cancer Sciences, Faculty of Medicine, University of Southampton, Southampton, UK. ${ }^{666}$ Department of Gene Technology, Tallinn University of Technology, Tallinn, Estonia. ${ }^{667}$ Genetics and Genome Biology Program, SickKids Research Institute, The Hospital for Sick Children, Toronto, ON M5G 1X8, Canada. ${ }^{668}$ Departments of Neurosurgery and Hematology and Medical Oncology, Winship Cancer Institute and School of Medicine, Emory University, Atlanta, GA 30322, USA. ${ }^{669}$ Department of Clinical and Molecular Medicine, Faculty of Medicine and Health Sciences, Norwegian University of Science and Technology, Trondheim, Norway. ${ }^{670}$ Argmix Consulting, North Vancouver, BC, Canada. ${ }^{671}$ Department of Information Technology, Ghent University, Interuniversitair Micro-Electronica Centrum (IMEC), Ghent, Belgium. ${ }^{672}$ Nuffield Department of Surgical Sciences, John Radcliffe Hospital, University of Oxford, Oxford OX1 2JD, UK. ${ }^{673}$ Institute of Mathematics and Computer Science, University of Latvia, Riga, LV 1586, Latvia. ${ }^{674}$ Discipline of Pathology, Sydney Medical School, University of Sydney, Sydney, NSW 2006, Australia. ${ }^{675}$ Department of Applied Mathematics and Theoretical Physics, Centre for Mathematical Sciences, University of Cambridge, Cambridge 10027, UK. ${ }^{676}$ Department of Statistics, Columbia University, New York, NY, USA. ${ }^{677}$ Department of Immunology, Genetics and Pathology, Science for Life Laboratory, Uppsala University, 75236 Uppsala, Sweden. ${ }^{678}$ Department of Histopathology, Cambridge University Hospitals NHS Foundation Trust, Cambridge CB2 OQQ, UK. ${ }^{679}$ Oxford NIHR Biomedical Research Centre, University of Oxford, Oxford OX1 2JD, UK. ${ }^{680}$ Georgia Regents University Cancer Center, Augusta, GA 30912, USA. ${ }^{681}$ Wythenshawe Hospital, Manchester M23 9LT, UK. ${ }^{682}$ Wellcome Centre for Human Genetics, University of Oxford, Oxford OX1 2JD, UK. ${ }^{683}$ Thoracic Oncology Laboratory, Mayo Clinic, Rochester, MN 55905, USA. ${ }^{684}$ Institute for Genomic Medicine, Nationwide Children's Hospital, Columbus, OH 43205, USA. ${ }^{685}$ Department of Obstetrics and Gynecology, Division of Gynecologic Oncology, Mayo Clinic, Rochester, MN 55905, USA. ${ }^{686}$ International Institute for Molecular Oncology, Poznań 60-203, Poland. ${ }^{687}$ Poznan University of Medical Sciences, 61-701 Poznań, Poland. ${ }^{688}$ Genomics and Proteomics Core Facility High Throughput Sequencing Unit, German Cancer Research Center (DKFZ), 69120 Heidelberg, Germany. ${ }^{689}$ NCCS-VARI Translational Research Laboratory, National Cancer Centre Singapore, Singapore 169610, Singapore. ${ }^{690}$ Edison Family Center for Genome Sciences and Systems Biology, Washington University, St. Louis, MO 63130, USA. ${ }^{691}$ MRC-University of Glasgow Centre for Virus Research, Glasgow G61 1QH, UK. ${ }^{692}$ Department of Medical Informatics and Clinical Epidemiology, Division of Bioinformatics and Computational Biology, OHSU Knight Cancer Institute, Oregon Health and Science University, Portland, OR 97239, USA. ${ }^{693}$ School of Electronic Information and Communications, Huazhong University of Science and Technology, 430074 Wuhan, China. ${ }^{694}$ Department of Applied Mathematics and Statistics, Johns Hopkins University, Baltimore, MD 21218, USA. ${ }^{695}$ Department of Cancer Genome Informatics, Graduate School of Medicine, Osaka University, Osaka 565-0871, Japan. ${ }^{696}$ School of Mathematics and Statistics, University of Sydney, Sydney, NSW 2006, Australia. ${ }^{697}$ Ben May Department for Cancer Research and Department of Human Genetics, University of Chicago, Chicago, IL 60637, USA. ${ }^{698}$ Tri-Institutional PhD Program in Computational Biology and Medicine, Weill Cornell Medicine, New York, NY 10065, USA. ${ }^{699}$ Department of Medicine and Therapeutics, The Chinese University of Hong Kong, Shatin, NT, Hong Kong, China. ${ }^{700}$ Department of Biostatistics, The University of Texas MD Anderson Cancer Center, Houston, TX 77030, USA. ${ }^{701}$ Duke-NUS Medical School, Singapore 169857, Singapore. ${ }^{702}$ Department of Surgery, Ruijin Hospital, Shanghai Jiaotong University School of Medicine, Shanghai, China. ${ }^{703}$ School of Computing Science, University of Glasgow, Glasgow G12 8QQ, UK. ${ }^{704}$ Division of Orthopaedic Surgery, Oslo University Hospital, 0450 Oslo, Norway. ${ }^{705}$ Eastern Clinical School, Monash University, Melbourne, VIC 3800 , Australia. ${ }^{706}$ Epworth HealthCare, Richmond, VIC 3121, Australia. ${ }^{707}$ Department of Biostatistics and Computational Biology, Dana-Farber Cancer Institute and Harvard Medical School, Boston, MA 02215, USA. ${ }^{708}$ Department of Biomedical Informatics, College of Medicine, The Ohio State University, Columbus, OH 43202, USA. ${ }^{709}$ The Ohio State University Comprehensive Cancer Center (OSUCCC-James), Columbus, OH 43202, USA. ${ }^{710} \mathrm{BIOPIC}$, ICG and College of Life Sciences, Peking University, 100871 Beijing, China. ${ }^{711}$ The University of Texas School of Biomedical Informatics (SBMI) at Houston, Houston, TX 77030, USA. ${ }^{712}$ Department of Biostatistics, University of North Carolina at Chapel Hill, Chapel Hill, NC 27599, USA. ${ }^{713}$ Department of Biochemistry and Molecular Genetics, Feinberg School of Medicine, Northwestern University, Chicago, IL 60208, USA. ${ }^{714}$ Faculty of Medicine and Health, University of Sydney, Sydney, NSW 2006, Australia. ${ }^{715}$ Department of Pathology, Erasmus Medical Center Rotterdam, 1066 CX Rotterdam, GD, The Netherlands. ${ }^{716}$ Division of Molecular Carcinogenesis, The Netherlands Cancer Institute, 1066 CXAmsterdam, The Netherlands.

${ }^{717}$ Institute of Molecular Life Sciences and Swiss Institute of Bioinformatics, University of Zurich, 8006 Zurich, Switzerland. 